Project No. 09-812

\title{
Multi-Scale Multi-physics Methods Development for the Calculation of Hot-Spots in the NGNP
}

Reactor Concepts RD\&D

Dr. Thomas Downar University of Michigan

Rob Versluis, Federal POC Hans Gougar, Technical POC

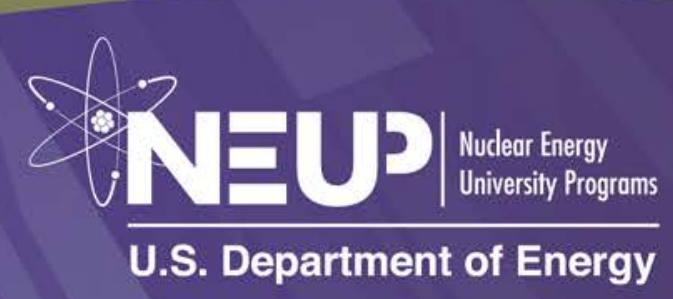




\section{FINAL REPORT}

Project Title:

Covering Period:

Date of Report:

Recipient:

Contract Number:

Project Number:

Principal Investigators: Thomas Downar- downar@umich.edu

Volkan Seker - vseker@umich.edu
Multi-Scale Multi-physics Methods Development for the Calculation of Hot-Spots in the NGNP

Final

April 30, 2013

University of Michigan

2355 Bonisteel Blvd

Ann Arbor, Ml 48109-2104

89536

$09-812$
Project Objective:

TPOCS:

Federal POC:

Colloborators:
The objective of the work here is to improve the accuracy in the prediction of local "hot" spots in the NGNP by developing multi-scale, multi-physics methods and implementing them within the framework of established codes used for NGNP analysis.

Hans Gougar - $\quad$ hans.gougar@inl.gov

Robert Versluis - rob.versluis@nuclear.energy.gov

William Martin, University of Michigan 


\section{Chapter 1 Introduction}

The following report summarizes the work performed in the project "Multi-Scale Multi-physics Methods Development for the Calculation of Hot-Spots in the NGNP". The report is separated into sequence of Chapters describing work performed on each phase of the project as identified in the original Milestone Chart:

\begin{tabular}{|c|c|c|c|}
\hline Milestone & Duration & Start Date & End Date \\
\hline I. VHTR Development & $\begin{array}{l}515 \\
\text { days }\end{array}$ & $10 / 5 / 2009$ & $9 / 23 / 2011$ \\
\hline I.1. VHTR Modeling & $\begin{array}{l}180 \\
\text { days }\end{array}$ & $10 / 5 / 2009$ & $6 / 11 / 2010$ \\
\hline I.1.1. Neutronic Models & 65 days & $10 / 5 / 2009$ & $1 / 1 / 2010$ \\
\hline I.1.1.a. PARCS Models & 25 days & $10 / 5 / 2009$ & $11 / 6 / 2009$ \\
\hline I.1.1.b MCNP Models & 30 days & $11 / 9 / 2009$ & $12 / 18 / 2009$ \\
\hline I.1.1.c. Validation and Consistency Studies & 30 days & $11 / 23 / 2009$ & $1 / 1 / 2010$ \\
\hline I.1.2. Thermal Hydraulic Modeling & $\begin{array}{c}115 \\
\text { days }\end{array}$ & $1 / 4 / 2010$ & $6 / 11 / 2010$ \\
\hline I.1.2.a. CFD Models & 70 days & $1 / 4 / 2010$ & $4 / 9 / 2010$ \\
\hline I.1.2.b. Validation and Consistency Studies & 45 days & $4 / 12 / 2010$ & $6 / 11 / 2010$ \\
\hline I.2. Methods Development & $\begin{array}{l}180 \\
\text { days }\end{array}$ & $10 / 5 / 2009$ & $6 / 11 / 2010$ \\
\hline I.2.1. PARCS methods for Prismatic Geometry & $\begin{array}{l}120 \\
\text { days }\end{array}$ & $10 / 5 / 2009$ & $3 / 19 / 2010$ \\
\hline I.2.2. Advanced MCNP/CFD Coupling Framework & 90 days & $2 / 8 / 2010$ & $6 / 11 / 2010$ \\
\hline I.2.3. In-line Doppler Treatment & 90 days & $11 / 16 / 2009$ & $3 / 19 / 2010$ \\
\hline I.2.4. Multgroup albedo treatment & 60 days & $3 / 22 / 2010$ & $6 / 11 / 2010$ \\
\hline I.3. Meso scale coupled calculation methods & $\begin{array}{l}195 \\
\text { days }\end{array}$ & $6 / 14 / 2010$ & $3 / 11 / 2011$ \\
\hline I.3.1. MCNP/CFD Interfacing & 55 days & $6 / 14 / 2010$ & $8 / 27 / 2010$ \\
\hline I.3.2. Coupled Calculations & 75 days & $8 / 30 / 2010$ & $12 / 10 / 2010$ \\
\hline Annual Report (Progress Report 4) & 15 days & $12 / 13 / 2010$ & $12 / 31 / 2010$ \\
\hline I.3.3. Boundary Condition Methods & 65 days & $12 / 13 / 2010$ & $3 / 11 / 2011$ \\
\hline I.4. Multiscale Calculations & 25 days & $3 / 14 / 2011$ & $4 / 15 / 2011$ \\
\hline I.5. Final Validation and Testing of Methods & $\begin{array}{l}115 \\
\text { days }\end{array}$ & $4 / 18 / 2011$ & $9 / 23 / 2011$ \\
\hline I.5.1. Neutronics Methods & 55 days & $4 / 18 / 2011$ & $7 / 1 / 2011$ \\
\hline I.5.2. Thermo-fluids & 35 days & $7 / 4 / 2011$ & $8 / 19 / 2011$ \\
\hline Annual Report (Progress Report 8) & 25 days & $8 / 22 / 2011$ & $9 / 23 / 2011$ \\
\hline II. HTR Development & $\begin{array}{l}200 \\
\text { days }\end{array}$ & $9 / 26 / 2011$ & $6 / 29 / 2012$ \\
\hline II.1. HTR Modeling & 135 & $9 / 26 / 2011$ & $3 / 30 / 2012$ \\
\hline
\end{tabular}




\begin{tabular}{|c|c|c|c|}
\hline & days & & \\
\hline II.1.1. Neutronic Models & 85 days & $9 / 26 / 2011$ & $1 / 20 / 2012$ \\
\hline II.1.1.a. PARCS Models & 25 days & $9 / 26 / 2011$ & $10 / 28 / 2011$ \\
\hline II.1.1.b. MCNP Models & 30 days & $10 / 31 / 2011$ & $12 / 9 / 2011$ \\
\hline II.1.1.c. Validation and Consistency Studies & 30 days & $12 / 12 / 2011$ & $1 / 20 / 2012$ \\
\hline II.1.2. Thermal Hydraulic Modeling & 50 days & $1 / 23 / 2012$ & $3 / 30 / 2012$ \\
\hline II.1.2.a. CFD Models & 30 days & $1 / 23 / 2012$ & $3 / 2 / 2012$ \\
\hline II.1.2.b. Validation and Consistency Studies & 20 days & $3 / 5 / 2012$ & $3 / 30 / 2012$ \\
\hline II.2. Boundary Condition Methods & 15 days & $4 / 2 / 2012$ & $4 / 20 / 2012$ \\
\hline II.3. Multiscale Calculations & 10 days & $4 / 23 / 2012$ & $5 / 4 / 2012$ \\
\hline II.4. Final Validation and testing of Methods & 40 days & $5 / 7 / 2012$ & $6 / 29 / 2012$ \\
\hline II.4.1. Neutronics Methods & 25 days & $5 / 7 / 2012$ & $6 / 8 / 2012$ \\
\hline II.4.2. Thermo-fluids & 15 days & $6 / 11 / 2012$ & $6 / 29 / 2012$ \\
\hline III. Documentation Preparation & 55 days & $7 / 2 / 2012$ & $9 / 14 / 2012$ \\
\hline III.1. Completion of SQA and Code Documentation & 55 days & $7 / 2 / 2012$ & $9 / 14 / 2012$ \\
\hline Final Report (Progress Report 12) & 50 days & $7 / 2 / 2012$ & $9 / 7 / 2012$ \\
\hline
\end{tabular}

The next Chapter describes fundamental multiscale neutronics research which was performed during the first year on methods to describe the interface between higher order multigroup neutron transport and the lower order few neutron diffusion theory which is used in standard NGNP calculations. Because a higher order nonorthogonal deterministic transport method was not available, this work was first performed in Cartesian geometry and the results were then later applied to the general geometry of the NGNP using Monte Carlo methods.

The third chapter describes the multiphysics methods which were developed in the second phase of the projects to provide the framework for the application of the work to practical NGNP reactor analysis. Specifically, the theory and methods implemented in the network flow, high temperature gas reactor dynamics code AGREE is described, to include the parallel development of higher order Computational Fluids Dynamics and Heat Transfer models for the simulation of local fluids and heat transfer in the fuel block.

The fourth chapter describes the development of the multiscale neutronics methods for non-orthogonal geometries of the prismatic NGNP. Specifically, the methods are discussed in which Monte Carlo methods are used to predict of the local pin power based on the deterministic nodal solution. The final chapter provides an overall summary, conclusions, and suggestions for future work. 


\section{Chapter 2 Multiscale Neutronics Methods}

The ability to accurately predict local pin powers in nuclear reactors is necessary to understand the mechanisms that cause fuel pin failure during steady state and transient operation. In the research here methods are developed to provide detailed higher order information locally with boundary conditions from a low order global solution. Several different core configurations were tested to determine the improvement in the local pin powers compared to the standard techniques which use diffusion theory and pin power reconstruction (PPR). Two different multiscale methods were developed and analyzed; the post-refinement multiscale method and the embedded multiscale method. The post-refinement multiscale methods use the global solution to determine boundary conditions for the local solution. The local solution is solved using either a fixed boundary source or an albedo boundary condition and the local solution is "postrefinement" and thus has no impact on the global solution. The embedded multiscale method allows the local solver to change the global solution to provide an improved global and local solution. The post-refinement multiscale method is assessed using three core designs. When the local solution has more energy groups the fixed source method has some issues near the interface; however the albedo method works well for all cases. In order to remedy the issue with boundary condition errors for the fixed source method, a buffer region is used to act as a filter which decreases the sensitivity of the solution to the boundary condition. Both the albedo and fixed source methods benefit from the use of a buffer region.

Unlike the post-refinement method, the embedded multiscale method alters the global solution. The ability to change the global solution allows for refinement in areas where the errors in the few groups nodal diffusion are typically large. The embedded method is shown to improve the global solution when it is applied to a MOX/LEU assembly interface, the fuel/reflector interface, and assemblies where control rods are inserted. The embedded method allows for multiple solution levels to be applied in a single calculation. The addition of intermediate levels to the solution improves the accuracy of the method. Both multiscale methods considered here have benefits and drawbacks but both can provide improvements over the current PPR methodology.

\subsection{Introduction}

The determination of the neutron flux distribution in nuclear reactors is crucial for both safety and economic reasons. The neutron flux is directly proportional to the power produced in the fuel. The ability to accurately predict the power distribution inside the core is important in determining the cooling requirements of the reactor during steady state operation and all hypothesized transients, including severe accidents. Economically, the ability to predict the pin power distribution will allow for better utilization of the fuel and decrease the probability of fuel 
failures during overpower events. These requirements put a heavy burden on the codes responsible for predicting the neutron flux throughout the reactor.

Standard practice in the design and analysis of nuclear reactors is the use of nodal diffusion codes coupled to one dimensional thermal-fluid codes. Such coupled code systems have the ability to accurately predict the average assembly powers. Pin power reconstruction methods have been developed to modulate the pin power distribution for a single assembly lattice calculation onto the full core solution. The efficiency of nodal diffusion codes makes them ideal to evaluate the neutron flux during both operational and accident analysis. Unfortunately, the underlying assumptions of diffusion theory prevent a detailed power distribution to be known with sufficient accuracy for complex geometries or in challenging transient conditions. For this reason, interest in the development of advanced codes which are capable of solving the Boltzmann transport equation throughout the core has increased.

These full core transport solvers have the ability to obtain a detailed distribution of pin powers throughout the core but are very computationally intensive. Full core transport solvers generally require the use of a computer cluster in order to hold the full problem domain in memory and solve the flux distribution in a reasonable time. The ability to solve full core transport transient problems is even more computationally intensive, in some cases requiring weeks of wall time for a few seconds of computation.

An important advantage that full core transport solvers have over the standard nodal diffusion codes is the ability to more accurately describe the effects of neighboring assemblies. In nuclear reactors there are several locations where the effect of neighboring assemblies can introduce errors into the calculation: the fuel-reflector interface, the insertion of control rods, and the interface of different fuel types. In the standard few group nodal diffusion methods the homogenized cross sections are generated using an infinite medium neutron spectrum which can be very different from the conditions in a modern core which can have very different fuel types and compositions. A basic assumption of current methods is the validity of an asymptotic spectrum to generate homogeneous cross sections, which has become less valid in highly heterogeneous fuel loadings currently used in industry.

One of the most common locations where diffusion theory does not work well is the fuelreflector interface. For assemblies facing the reflector, the assumption of an infinite assembly does not accurately describe the correct energy spectrum inside the assembly. The reflector allows for a large leakage of high energy neutrons and a source of thermal energy neutrons which cause fission. The ability to resolve this interface requires special treatment during modeling. The most common practice is to use a two assembly calculation which is comprised of the fuel assembly and a reflector region. In order to preserve the net current at the interface a discontinuity factor is defined. Although this methodology is practical and improves the calculation of the fuel-reflector interface, the method is not robust. Generally, this type of 
calculation is only performed for a single fuel type with zero burnup, whereas, there are several fuel types that touch a boundary and the burnup associated with the peripheral fuel is also not uniform. Another very common location where diffusion theory introduces errors is in the vicinity of control rods. The introduction of control rods causes a very sharp drop in the thermal neutron flux. Although the core solution does a good job of approximating the worth of a control rod, the pin power distribution in neighboring regions can have significant errors. The ability to calculate the pin power around control rods accurately can be very important when predicting the fuel performance during events such as a control rod ejection. In this accident, the fuel pins facing the control rod can experience a higher deposition of energy then the assembly average. Finally, another common region where diffusion theory introduces errors is at the interface of unlike fuel types. With the interest in burning weapons grade Plutonium in nuclear reactors in recent years; interest has grown in the ability to load mixed oxide (MOX) fuel into operating nuclear reactors. The addition of these assemblies into the nuclear reactors creates a very heterogeneous system of standard low enriched Uranium (LEU) assembly and MOX assemblies. The computational issues with these simulations are the fact that the energy spectrum of an LEU assembly and a MOX assembly can be very different. The existence of Plutonium in the MOX assemblies adds a large absorption resonance below $1 \mathrm{eV}$ which does not exist in standard LEU fuel. The LEU-MOX interface can be a significant source of error in nodal diffusion calculations and a special treatment must be used in order to minimize the errors in the standard homogenized nodal methods.

The error in few group nodal diffusion in each of the cases noted above is directly attributable to localized effects. The objective of this work is to increase the resolution of the solver locally where errors are introduced by the few group diffusion approximation but still utilize the nodal diffusion solver globally. This is achieved through two different methods. The first method is an a posteriori method where the global solution is used to determine the boundary conditions for a local problem. This method does not provide any feedback to the global solver and will be referred to as "one way coupling". The second method is an embedded calculation where the global and local solutions are solved simultaneously to provide a consistent solution. The second method is much more consistent with traditional adaptive multiscale methods and will be referred to as "two way coupling".

\subsection{Background on Multiscale Neutronics Methods}

Multiscale methods provide the framework to perform detailed calculation locally while still relying on a coarser solution globally. The essential idea behind the multiscale method is to decompose the solution into suitable scales; macro, meso, micro, etc. The objective of these scales is to increase fidelity where important physics is occurring or the mathematical model of the macroscale is insufficient to capture the full physics. The goal is to optimize the computational cost of the multiscale scheme with the cost of a detailed global solution. 


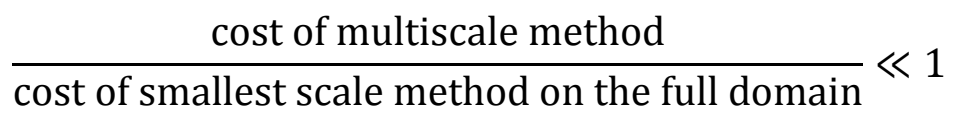

One of the classical examples of the multiscale method is the multigrid method. In this method, the spatial grid is refined in order to decrease the discretization error. The grid refinement can be done on a global scale (full multigrid) and the multigrid scheme simply accelerates the solution of the smallest scale or it can be done on a local scale (adaptive multigrid) where the grid is reduced where the discretization error is large. The standard coarse mesh finite difference (CMFD) procedure is an example of a full multigrid method.

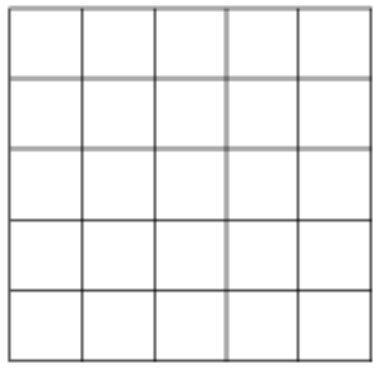

(a) Level 1

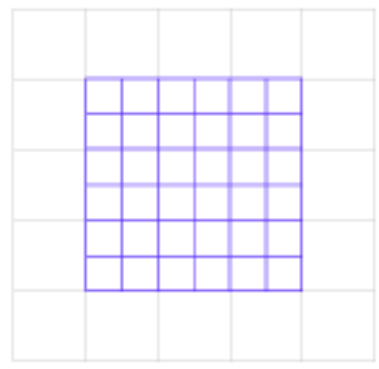

(b) Level 2

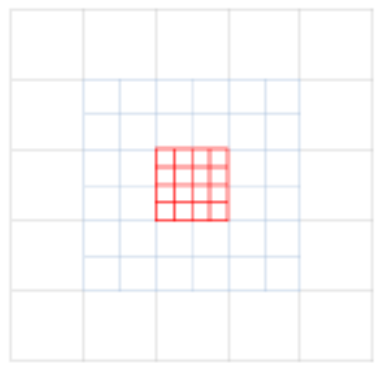

(c) Level 3

Figure 1.1 Composite grids corresponding to each level

The refinement of multiscale methods is performed adaptively and can be done either statically or dynamically. Static adaptive methods determine how to refine the grid using a predefined map, generally defined by the user. Dynamic adaptive methods, on the other hand, use criteria of the solution to determine if the grid should be refined. The criteria used can vary depending on the physics of interest. In principle, the two types of adaptive methods can be combined. In this case, the user would specify a location of interest and the rest of the domain would be refined dynamically. However, the focus of the work here is on static adaptive methods. The adaptive multiscale method relies on a hierarchy of grids and methods. The first level represents the global domain with the macro-scale method. As the grid level increases, the grid is restricted to smaller and smaller subdomains and the method also increases in fidelity. Figure 1.1 shows the composite grid. Level 1 contains a coarse representation of the global domain. Level 2 and 3 refine the grid and the method. The remainder of the coarse grid is depicted but the grayed out and is not used during the computation of the level. In addition to determining and defining the levels, it is necessary to define an iteration strategy between the levels. There are several iteration strategies for maneuvering between levels. The $\mathrm{V}$ cycle iteration is a basic iteration strategy which starts at level 1 and gradually increases the levels until the maximum number of levels is reached and then gradually reduces the level back to 1 . This type of iteration scheme is repeated until all levels reach their convergence as shown in Figure 1.2. Another iteration scheme, the $\mathrm{W}$ cycle, strives to limit the number of calls to the higher 
levels until the lower levels reach a certain convergence criteria. This iteration strategy is depicted in Figure 1.3.

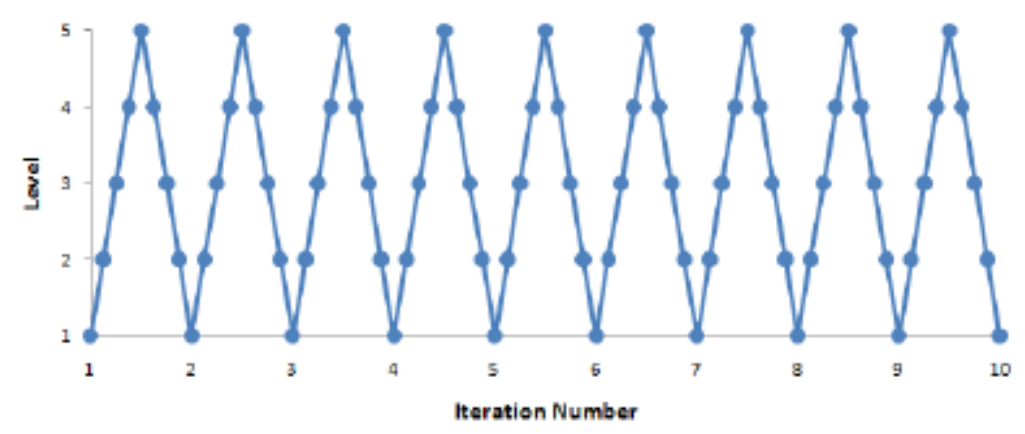

Figure 1.2 Traditional V-Cycle Iteration Strategy

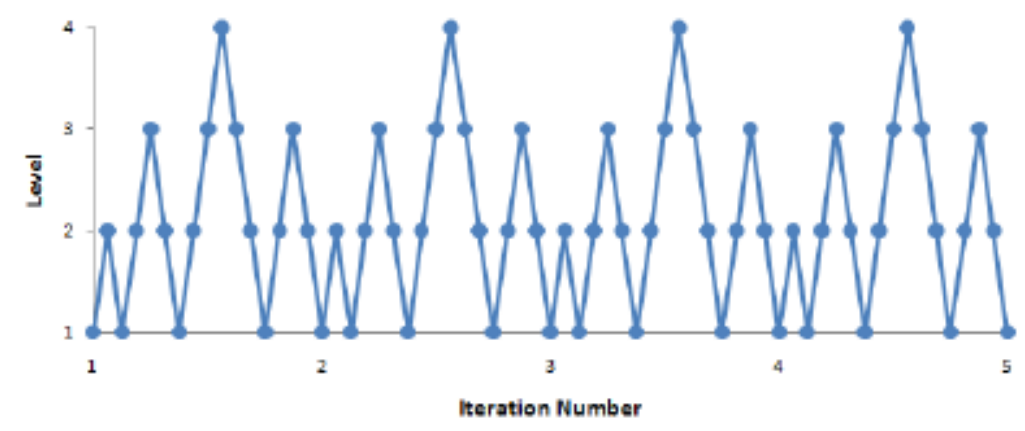

Figure 1.3 W-Cycle Iteration Strategy with Multiple Levels

\section{a. Neutron Transport Theory}

The steady-state neutron transport equation describes how neutrons stream, are lost through collision, and are born into six dimensional phase space as shown in equation 2 .

$$
\begin{aligned}
\boldsymbol{\Omega} \cdot \boldsymbol{\nabla} \psi(\boldsymbol{x}, \boldsymbol{\Omega}, E) & +\Sigma_{t}(\boldsymbol{x}, E) \psi(\boldsymbol{x}, \boldsymbol{\Omega}, E) \\
=\frac{\chi(E)}{4 \pi k} \int_{4 \pi} \int_{0}^{\infty} \nu \Sigma_{f}\left(\boldsymbol{x}, E^{\prime}\right) \psi\left(\boldsymbol{x}, \boldsymbol{\Omega}^{\prime}, E^{\prime}\right) d E^{\prime} d \Omega^{\prime} & \\
& \quad+\int_{4 \pi} \int_{0}^{\infty} \Sigma_{\boldsymbol{s}}\left(\boldsymbol{x}, \boldsymbol{\Omega}^{\prime} \cdot \boldsymbol{\Omega}, E^{\prime} \rightarrow E\right) \psi\left(\boldsymbol{x}, \boldsymbol{\Omega}^{\prime}, E^{\prime}\right) d E^{\prime} d \Omega^{\prime}
\end{aligned}
$$

Eq. 2

$\Psi$ is the angular neutron distribution in space $(\mathrm{x})$, angle $(\Omega)$, and energy $(\mathrm{E}) . \sum_{\mathrm{x}}$ is the macroscopic cross-section of type " $x$ " which is total (t), fission (f), or scattering (s). $v$ is the average number of neutrons generated per fission, $\chi(\mathrm{E}) \mathrm{dE}$ is the probability that fission neutrons are created in $\mathrm{dE}$ about energy $\mathrm{E}$. $\mathrm{k}$ is the k-eigenvalue (multiplication factor) of the system. The 
k-eigenvalue modifies the number of neutrons produced by each fission to preserve the global balance of neutrons. The transport equation is complicated by the energy dependent coefficients and cross-sections, as shown in Figure 2.1.

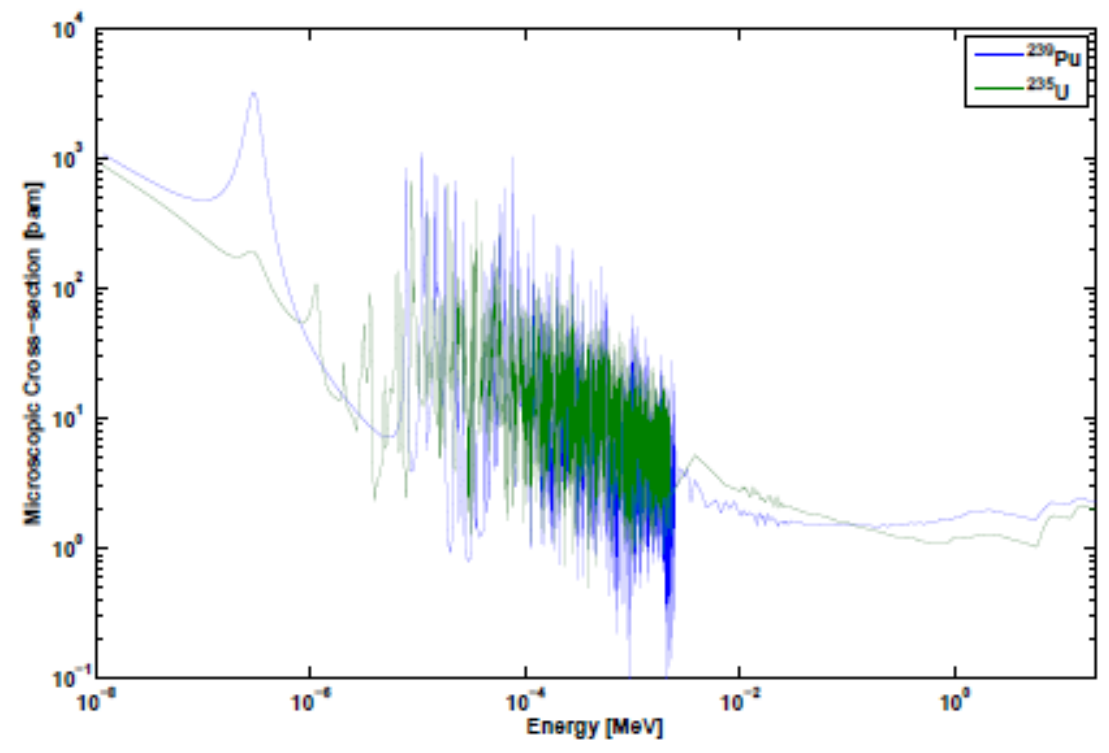

Figure 2.1: ${ }^{235} \mathrm{U}$ and ${ }^{239} \mathrm{Pu}$ Total Cross-sections

The complexities of the neutron transport equation make it very difficult to solve for full core problems. Several methods have been developed over the past decades to efficiently model standard reactor designs. Solutions of the transport equation can be broken up into two categories: deterministic and stochastic. Deterministic methods discretize each of the free variables introducing discretization error into the solution. Stochastic methods model the random nature of the transport equation and thus do not have discretization errors but instead introduce statistical errors.

Deterministic methods require the spatial, angular, and energy domain to be discretized. The spatial domain is generally broken into finite volumes in which the transport equation is solved. The exact method of treating the spatial variables differs between different methods. The angular variable is generally treated in two ways. The first method is to discretize the angle across the unit sphere. The second method is to solve for integral moments of the angular variable. Almost all deterministic methods are dependent on the multigroup method to treat the energy variable.

The multigroup method defines energy groups on which an average cross-section is defined for each group. The average cross-section is defined by preserving the reaction rate inside the energy group. 


$$
\begin{aligned}
\psi_{g}(\boldsymbol{x}, \boldsymbol{\Omega}) & =\int_{E_{g}}^{E_{g-1}} \psi(\boldsymbol{x}, \boldsymbol{\Omega}, E) d E \\
\Sigma_{x, g}(\boldsymbol{x}, \boldsymbol{\Omega}) & =\frac{\int_{E_{g}}^{E_{g-1}} \Sigma_{x}(\boldsymbol{x}, E) \psi(\boldsymbol{x}, \boldsymbol{\Omega}, E) d E}{\psi_{g}(\boldsymbol{x}, \boldsymbol{\Omega})} \\
\chi_{g}(\boldsymbol{x}) & =\int_{E_{g}}^{E_{g-1}} \chi(\boldsymbol{x}, E) d E
\end{aligned}
$$

Generally another assumption is made that the angular flux is separable in energy and angle. This simplifies the definition of the multigroup cross-sections to only be a function of space.

$$
\Sigma_{x, g}(\boldsymbol{x})=\frac{\int_{E_{g}}^{E_{g-1}} \Sigma_{x}(\boldsymbol{x}, E) \phi(\boldsymbol{x}, E) d E}{\phi_{g}(\boldsymbol{x})}
$$

These definitions yield the multigroup transport equation.

$$
\begin{aligned}
\boldsymbol{\Omega} \cdot \boldsymbol{\nabla} \psi_{g}(\boldsymbol{x}, \boldsymbol{\Omega})+\Sigma_{t, g}(\boldsymbol{x}) \psi_{g}(\boldsymbol{x}, \boldsymbol{\Omega}) & \\
=\frac{\chi_{g}}{4 \pi k} \sum_{g^{\prime}=1}^{G} \int_{4 \pi} \nu \Sigma_{f, g^{\prime}}(\boldsymbol{x}) \psi_{g^{\prime}}\left(\boldsymbol{x}, \boldsymbol{\Omega}^{\prime}\right) d \Omega^{\prime} & \\
& \quad+\sum_{g^{\prime}=1}^{G} \int_{4 \pi} \Sigma_{\boldsymbol{s}, g^{\prime} \rightarrow g}\left(\boldsymbol{x}, \boldsymbol{\Omega}^{\prime} \cdot \boldsymbol{\Omega}\right) \psi_{g^{\prime}}\left(\boldsymbol{x}, \boldsymbol{\Omega}^{\prime}\right) d \Omega^{\prime}
\end{aligned}
$$

The principal problem with the multigroup equations is the need to know the continuous energy flux to correctly weight the continuous energy cross-sections. Several methods have been proposed to calculate multigroup cross-sections but the two major methods are assume an analytic shape to the flux or calculate the point-wise continuous flux . Generally a combination of these two methods are used; analytic expressions where the flux solution is straightforward and well known and point wise continuous numerical solution in regions where the flux solution is non-trivial such as the resonance region. The numerical solutions are generally computed on a significantly reduced spatial domain which generally is zero or one dimensional in nature. Another common assumption is to rewrite the differential scattering cross-section as an infinite Legendre expansion.

$$
\psi_{g}(\boldsymbol{x}, \boldsymbol{\Omega})=\int_{\substack{E_{g} \\-E}}^{E_{g-1}} \psi(\boldsymbol{x}, \boldsymbol{\Omega}, E) d E
$$

The differential scattering can normally be well approximated by truncating the infinite sum after the first or second moment. In this work isotropic scattering, $\mathrm{P}_{0}$, will be assumed for simplicity. The resulting multigroup transport equation with isotropic scattering can be written as follows. 


$$
\begin{aligned}
\boldsymbol{\Omega} \cdot \boldsymbol{\nabla} \psi_{g}(\boldsymbol{x}, \boldsymbol{\Omega})+\Sigma_{t, g}(\boldsymbol{x}) \psi_{g}(\boldsymbol{x}, \boldsymbol{\Omega}) \\
\quad=\frac{\chi_{g}}{4 \pi k} \sum_{g^{\prime}=1}^{G} \nu \Sigma_{f, g^{\prime}}(\boldsymbol{x}) \phi_{g^{\prime}}(\boldsymbol{x})+\frac{1}{4 \pi} \sum_{g^{\prime}=1}^{G} \Sigma_{\boldsymbol{s} 0, g^{\prime} \rightarrow g}(\boldsymbol{x}) \phi_{g^{\prime}}(\boldsymbol{x})
\end{aligned}
$$

$\Phi$ is the scalar flux which is simply the angle integrated angular flux .

$$
\phi_{g}(\boldsymbol{x})=\int_{4 \pi} \psi_{g}(\boldsymbol{x}, \Omega) d \Omega
$$

Nodal diffusion codes have been the workhorse of reactor design and analysis for the past several decades. The nodal diffusion method is dependent on the diffusion form of the transport equation.

\section{b. Diffusion Theory}

The diffusion equation derived from the $\mathrm{P}_{\mathrm{n}}$ class of methods which expand moments of the angular flux using Legendre polynomials.

$$
\psi_{g}(\boldsymbol{x}, \boldsymbol{\Omega})=\sum_{n=0}^{\infty} \frac{2 n+1}{4 \pi} P_{n}(\boldsymbol{\Omega}) \phi_{g, n}(\boldsymbol{x})
$$

The $\mathrm{P}_{1}$ equation is obtained by taking the zeroth and first angular moment of the transport equation.

$$
\begin{gathered}
\nabla \cdot \boldsymbol{J}_{g}(\boldsymbol{x})+\Sigma_{t, g}(\boldsymbol{x}) \phi_{g}(\boldsymbol{x})=\sum_{g^{\prime}=1}^{G}\left(\frac{\chi g}{k} \nu \Sigma_{f, g^{\prime}}(\boldsymbol{x})+\Sigma_{s 0, g^{\prime} \rightarrow g}(\boldsymbol{x})\right) \phi_{g^{\prime}}(\boldsymbol{x}) \\
\frac{1}{3} \boldsymbol{\nabla} \phi_{g}(\boldsymbol{x})+\Sigma_{t, g}(\boldsymbol{x}) \boldsymbol{J}_{g}(\boldsymbol{x})=\sum_{g^{\prime}=1}^{G} \Sigma_{s 1, g^{\prime} \rightarrow g}(\boldsymbol{x}) \boldsymbol{J}_{g^{\prime}}(\boldsymbol{x})
\end{gathered}
$$

The P1 equations are written solely in terms of the scalar flux $\Phi$ and the net neutron current $\mathrm{J}$ which are defined as follows.

$$
\begin{aligned}
\phi_{g}(\boldsymbol{x}) & =\int_{4 \pi} \psi_{g}(\boldsymbol{x}, \Omega) d \Omega \\
\boldsymbol{J}_{g}(\boldsymbol{x}) & =\int_{4 \pi} \Omega \psi_{g}(\boldsymbol{x}, \boldsymbol{\Omega}) d \Omega
\end{aligned}
$$

The diffusion equation and the P1 equations differ in two ways. The first is that the time derivative of the current is assumed to be zero for the diffusion equation. Since only the steady 
state equations are shown here, this approximation is exact. The second assumption made is that the anisotropic scattering term,_s1, can be simplified into a diagonal matrix. Since the scattering is assumed to be isotropic, this term is zero and this approximation becomes exact as well.

$$
\Sigma_{t r, g}(\boldsymbol{x})=\left(\Sigma_{t, g}(\boldsymbol{x})-\Sigma_{s 1, g, g^{\prime}}(\boldsymbol{x})\right)
$$

The diffusion equation is obtained by solving equation $(2.9 \mathrm{~b})$ for the current and substituting that into equation (2.9a).

$$
\begin{aligned}
-\frac{1}{3} \boldsymbol{\nabla} \cdot \Sigma_{t r, g}^{-1} \nabla \phi_{g}(x)+ & \Sigma_{t, g}(x) \phi_{g}(x) \\
& =\frac{\chi_{g}}{k} \sum_{g^{\prime}=1}^{G} \nu \Sigma_{f, g^{\prime}}(x) \phi_{g^{\prime}}(x)+\sum_{g^{\prime}=1}^{G} \Sigma_{s 0, g^{\prime} \rightarrow g}(x) \phi_{g^{\prime}}(x)
\end{aligned}
$$

Most nodal methods today are based on Equivalence theory proposed by Koebke [19] and extended to Generalized Equivalence theory by Smith [20]. Generalized Equivalence theory uses a three-step approach for determining the power distribution in the reactor. The first step is to generate the transport multigroup cross-section library using zero or one dimensional methods. The transport library is generally between 50 to 200 energy groups.

The second step is to perform a two-dimensional assembly-level calculation for each assembly type using the transport library. Using the solution of this model, the cross-sections are homogenized in space and used to generate a few group library which typically has 2-8 energy groups. Other parameters are also computed to enforce equivalence between the assembly and core level solutions. In many cases where thermal hydraulic feedback is required, the few group library is generated at different reference conditions (coolant density, fuel temperature, boron concentration, etc.).

In the third and final step, the nodal diffusion code uses the few group library to solve for the full core problem. The nodal diffusion code is normally coupled to one dimensional thermal hydraulic codes to provide the thermal hydraulic feedback in the core system.

\section{Assembly-level (Lattice) Computation}

The assembly-level computation relies on a code which is capable of solving the two dimensional transport equations. The single assembly model should accurately account for all of the geometric complexities in the lattice and have a fine enough spatial mesh to accurately resolve the angular flux distribution in the assembly. Several methods have been used to solve the assembly-level problem, many of which are discussed in the Transport Methods section. 


\section{Homogenization and Discontinuity Factors}

The spatial and energy flux distribution obtained from the assembly-level calculation is used to generate assembly homogenized few group cross-sections. The cross-sections are collapsed to preserve the average reaction rates.

$$
\bar{\Sigma}_{x, g}=\frac{\int \sum_{h \in g} \Sigma_{x, h}(\boldsymbol{x}) \phi_{h}(\boldsymbol{x}) d V}{\int \sum_{h \in g} \phi_{h}(\boldsymbol{x}) d V}
$$

where $\mathrm{h}$ is the fine group energy and $\mathrm{g}$ is the coarse group energy. The methodology to homogenize the transport cross-section is not as clear. Several methods have been proposed to homogenize the transport cross-section including flux weighting, inverse flux weighting, and current weighting. It has been found that flux weighting in space and inverse flux weighting in energy works best for reactor analysis.

Generalized Equivalence Theory provides an extra degree of freedom to match the assembly-level solution. This degree of freedom is captured in discontinuity factors. The discontinuity factor accounts for the loss of spatial resolution at the interface during homogenization. Multiple discontinuity factors can be specified depending on the number of free variables the nodal method can accept. Side discontinuity factors specify the ratio of the surface flux calculated by the assembly-level calculation and the surface flux predicted by the nodal method.

$$
S D F_{g, f}=\frac{\phi_{g, f}^{\text {Transport }}}{\phi_{g, f}^{\text {Diffusion }}}
$$

SDF is the side discontinuity factor, $\mathrm{g}$ is the few energy groups, and $\mathrm{f}$ is the surface. Other nodal methods can also utilize corner discontinuity factors which are the ratio of the point flux calculated by the assembly-level calculation and what the nodal method predicts.

$$
C D F_{g, c}=\frac{\phi_{g, c}^{\text {Transport }}}{\phi_{g, c}^{\text {Diffusion }}}
$$

CDF is the corner discontinuity factor and c represents the corner in which the flux is calculated. 
The last piece of information that is extracted from the assembly-level calculation is the groupwise pin power form function (GFF). The GFF allows the pin powers to be reconstructed in the entire core.

$$
P_{i, j}=\sum_{g=1}^{G} G F F_{g, i, j} \tilde{\phi}_{g, i, j}
$$

$\mathrm{GFF}_{\mathrm{g}, \mathrm{i}, \mathrm{j}}$ is the group form function for group $\mathrm{g}$ and pin position $\mathrm{i}, \mathrm{j}$ and $\Phi_{\mathrm{g}, \mathrm{i}, \mathrm{j}}$ is the reconstructed scalar flux from the homogeneous diffusion solution of the lattice. The GFF is computed by taking the ratio of the power generated by group $g$ as calculated using the assembly level calculation to the average pin flux as calculated by an equivalent diffusion model.

$$
G F F_{g, i, j}=\frac{\kappa \Sigma_{f, g, i, j} \phi_{g, i, j}^{\text {Transport }}}{\phi_{g, i, j}^{\text {Diffusion }}}
$$

\section{c. Core-level Computation}

Once the lattice physics code generates all the parameters needed, a core wide computation can be performed. The core computation starts with the spatial discretization of the core. In the radial direction the assembly size is typically used. In the axial direction a grid is typically set to $10-20 \mathrm{~cm}$. There are two common solutions to the core wide diffusion problem. The first is to use the diffusion equation and apply a fine mesh finite difference method. This requires a further internal discretization of each assembly which is typically made on a pin size level. The differential equation is then discretized using the finite difference approximation to solve the full core system.

A second method that is commonly used is a transverse leakage method. These methods convert the three dimensional differential equation into three one dimensional differential equations coupled through a transverse leakage term.

$$
\begin{gathered}
-D \frac{d^{2}}{d x_{i}^{2}} \phi\left(x_{i}\right)+\Sigma_{t} \phi\left(x_{i}\right)=Q\left(x_{i}\right)-L\left(x_{i}\right) \\
L\left(x_{i}\right)=l_{0}+l_{1} P_{1}\left(x_{i}\right)+l_{2} P_{2}\left(x_{i}\right)
\end{gathered}
$$


Where $\mathrm{L}$ (xi) is the transverse leakage sink and $\mathrm{Pn}$ (xi) is a function which defines the distribution of the leakage inside the node. Typically the transverse leakage if found by interpolating a shape function using the node average leakage of the current node and the neighboring nodes. Once the solution is cast into a one dimensional differential equation, the solution possibilities become much simpler. The first option is to expand the nodal flux and source using a polynomial basis set and solve for the node balance constraints (continuity of surface flux and current) as well as spatial moments of the differential equation. This method is known as the Nodal Expansion Method (NEM).

The second option is to solve for the analytic solution of the nodal flux (ANM). With one energy group, this solution is trivial, unfortunately for reactor problems a minimum of two groups is needed. For two group problems methods have been developed which use a spectral decomposition of the coupled differential equations to solve the groups simultaneously. For more energy groups, the spectral decomposition method is not as easy to derive. In this case, the system of equations can be written in such a way that solution becomes a matrix exponential. The matrix exponential can be solved using a variety of methods but can easily become illconditioned and can be difficult to implement boundary conditions.

The last option, bridges the gap between the NEM and ANM. This method solves the analytic solution to the differential equation in which the source is expanded using a polynomial. This method is known as the Semi-Analytic Nodal Method (SANM).

The group coupling is effectively removed from the analytic solution so groups do not need to be solved simultaneously. Once the analytic form of the flux is found, it is recast into a polynomial to update the source term. This method has advantages over the other two because the solution resembles the analytic result, reduces overshoots and undershoots from a pure polynomial method, and can be expanded to use an arbitrary number of groups. The main down side is the complication of implementation because the projection equations from analytic to polynomial can become quite complex.

All of the methods discussed above can be implemented with surface discontinuity factors. In some cases the corner discontinuity factors will be used in the calculation of the pin powers. The core calculation can produce very accurate results if the cross-sections and discontinuity factors are generated correctly.

\section{Nodal Fine Mesh Finite Difference}

The fine mesh finite difference method is a common computational method that is used for many numerical solutions to partial differential equations. The methodology is well known for classical diffusion equations and is very similar for neutron transport. Like the classical 
solutions the node average flux is assumed to be the centroid flux and the derivative of the flux needed to determine the neutron current is calculated using the first two terms of the Taylor expansion. There are two main differences in neutron diffusion theory that differ from classical diffusion problems (such as heat conduction). The first difference is the presence of the collision operator in the neutron diffusion equation. The second difference is the use of discontinuity factors at the assembly interface.

\section{Fine Mesh Finite Difference Formulation}

The FMFD method starts by integrating the diffusion equation over a rectangular region. The neutron current at the surface of each face is balanced by the neutron source minus collision losses in each node.

$$
\sum_{f=n, e, s, w}(\boldsymbol{J} \cdot \boldsymbol{A})_{f}+\Sigma_{P, t} \phi_{P} V=\sum_{g^{\prime}=1}^{G} \Sigma_{P, s, g^{\prime} \rightarrow g} \phi_{P, g^{\prime}} V+\frac{\chi_{P, g}}{k} \sum_{g^{\prime}=1}^{G} \nu \Sigma_{P, f, g^{\prime}} \phi_{P, g^{\prime}} V
$$

The flow of neutrons at the surfaces is written in two different equations describing how neutrons flow from the node average values to the surface, then from the surface to the neighboring node. At the interface the discontinuity factor is applied.

$$
\begin{gathered}
J_{e}=D_{P} \frac{\phi_{P}-\phi_{P, e}}{0.5 h_{P}} \\
J_{e}=D_{E} \frac{\phi_{E, w}-\phi_{E}}{0.5 h_{E}} \\
f_{P, e} \phi_{P, e}=f_{E, w} \phi_{E, w}
\end{gathered}
$$

These three constraints are applied to construct the net current at the interface as a linear combination of the centroid value and the centroid of the neighboring cell.

$$
J_{e}=\frac{2 D_{P} D_{E}\left(f_{P, e} \phi_{P}-f_{E, w} \phi_{E}\right)}{D_{E} f_{P, e} h_{P}+D_{P} f_{E, w} h_{E}}
$$


For the problems considered in this work the fine mesh is defined using the pin cell geometry but the side discontinuity factors are only defined on the assembly level.

In order to account for the difference in locations where the discontinuity factors are defined, the discontinuity factors are assumed to be unity for interior faces of the assembly and adopt the assembly surface discontinuity factor for external faces of the assembly. The corner discontinuity factors are not utilized in this method. Lastly the group form functions which allow for the pin powers to be reconstructed from the homogenized solution simply uses the node average flux which have been defined to be the average flux over the pin cell by nature of the FMFD method.

The last issue that must be addressed is the application of the boundary conditions of the global system. Generally the boundary condition specified is an albedo boundary. For most diffusion codes the albedo is defined slightly different than the methodology described in the previous chapter. Instead the $\alpha$ albedo is defined as the ratio of the net current and the surface flux for each energy group.

$$
\alpha_{g}=\frac{\boldsymbol{J}_{g, s} \cdot \boldsymbol{n}}{\phi_{g, s}}
$$

The definition of the albedo is substituted into the FMFD definition of the current to obtain a relationship between the boundary current and the node average flux.

$$
\begin{aligned}
& J_{e}=D_{P} \frac{\phi_{P}-\phi_{P, e}}{0.5 h_{P}} \\
& J_{e}=\alpha_{e} f_{P, e} \phi_{P, e} \\
& J_{e}=\frac{2 \alpha_{e} D_{P} f_{P, e} \phi_{P}}{2 D_{p}+\alpha_{e} f_{P, e} h_{P}}
\end{aligned}
$$

\section{Source Iteration}

Now that the diffusion equation is discretized and written in terms of the the average nodal flux and the average flux of the neighboring assemblies, the system of equations can be complied in matrix form. The matrix is of dimension $\mathrm{N}_{\text {node }} \mathrm{G} \times \mathrm{N}_{\text {node }} \mathrm{G}$ and can be written in operator form as follows. 


$$
\begin{gathered}
\mathbb{D} \phi+\mathbb{T} \phi=\mathbb{S} \phi+\frac{1}{k} \chi{\overrightarrow{\nu \vec{\nu}_{f}^{\top}} \phi}_{\mathbb{M}}^{\top}=\mathbb{D}+\mathbb{T}-\mathbb{S} \\
\mathbb{F}=\chi \overrightarrow{\nu \vec{\Sigma}_{f}^{\top}} \\
\mathbb{M} \phi=\frac{1}{k} \mathbb{F} \phi=\lambda \mathbb{F} \phi
\end{gathered}
$$

Where $\mathrm{D}$ contains a matrix relating all of the streaming terms, $\mathrm{T}$ is a diagonal matrix containing the total cross-section of each node and group, $\mathrm{S}$ is a block diagonal matrix containing the scattering matrix of each node, $\mathrm{F}$ is the fission operator which defines the fission source in each node, and $\lambda$ is the inverse of the multiplication factor. The final form of the operator form of the diffusion equation is an eigenvalue problem. Since the fundamental mode (largest multiplication factor) is the desired quantity for steady-state core analysis, the inverse power method is used to calculate the eigenvalue/eigenvector pair.

$$
\begin{gathered}
\mathbb{M} \phi^{k+1}=\lambda^{k} \mathbb{F} \phi^{k} \\
\lambda^{k+1}=\frac{\left\|\mathbb{F} \phi^{k+1}\right\|}{\left\|\mathbb{F} \phi^{k}\right\|}
\end{gathered}
$$

Since $\Phi^{\mathrm{k}}$ is known at the beginning of the $\mathrm{k}+1$ iteration, equation $(2.25 \mathrm{a})$ is of the following form.

$$
\mathbb{A} \boldsymbol{x}=\boldsymbol{b}
$$


This equation can be solved using a direct linear equation solver such as Gaussian elimination, an iterative solver such as Gauss-Siedel or Jacobi methods, or using a Krylov subspace method such as GMRES or BiCGstab. Several solvers were implemented in the code developed and various methods were tested to determine the best results. Ultimately the GMRES solver was determined to be the most efficient but the direct solver was determined to be the most stable.

\section{Wielandt Shift Acceleration}

The source iteration method is known to be slowly converging for cases with dominance ratios (the ratio of the largest $\mathrm{k}$ to the second largest $\mathrm{k}$ ) close to 1 . One method that can accelerate the convergence of the source iteration is to shift part of the fission source to the left hand side of the equation.

$$
\left(\mathbb{M}-\lambda_{\text {shift }} \mathbb{F}\right) \phi=\left(\lambda-\lambda_{\text {shift }}\right) \mathbb{F} \phi
$$

$\lambda$ shift is the amount of the fission source that is moved to the left hand side of the equation and the inverse power method is applied to the shifted system. Care must be taken when choosing the value of $\lambda$ shift. Since the inverse power method finds the eigenvalue closest to zero it is possible to overshift the system and converge to an eigenvalue which does not represent the fundamental mode. One method to specify $\lambda$ shift is to fix the value to be $1 / \mathrm{kmax}$ where $\mathrm{kmax}$ is the maximum multiplication factor that is possible ( 3 is the default kmax used because it represents the average number of neutrons born per fission). Another method to specify $\lambda$ shift is to allow the user to input a fixed value for the shifted eigenvalue. The last method changes the shifted eigenvalue as the solution iterates to obtain an optimum shift.

This is obtained by using the current estimate of the eigenvalue and the change in the eigenvalue between successive iterations.

$$
\lambda_{\mathrm{shift}}^{k+1}=\alpha_{0} \lambda^{k}-\alpha_{1}\left|\lambda^{k}-\lambda^{k-1}\right|
$$

$\alpha 0$ is a constant less than 1 which does not allow the full eigenvalue to be shifted otherwise the matrix would become singular at convergence and $\alpha 1$ is a constant which scales the error to prevent overshifting during the iteration. The values used in this work are $\alpha 0=0.95$ and $\alpha 1=5$.

\section{Transport Methods}

Limitations of nodal diffusion methods along with considerable advancements in computing technology are two of the main reasons that transport methods have found their way into reactor design and analysis. There are several classes of methods to solve the transport equation. This discussion will focus on a few of the major methods used to solve the full core 
transport equation. One of the most popular methods for solving full core transport is the discrete ordinates method (SN). The SN method assumes that the angular flux can be discretized into discrete angles each with a corresponding weight. These angles are defined by a quadrature set. The quadrature set for 1-D problems are almost universally the Gauss-Legendre sets but in 3-D problems there are many proposed quadrature sets. One of the most common quadrature sets for 3-D problems is the Level-Symmetric quadrature set. The other common method for solving the full core transport problem is the Method of Characteristics, MOC. In this method characteristic paths of flight are drawn across the domain with a user specified spatial and angular discretization.

The transport equation is then integrated across these characteristic directions. The computational burden for the application of 3-D MOC methods to core problems is generally prohibitive for full core calculations. Approximate "2D-1D" methods have been developed [21] that are based on the assumption that the solution is separable in the radial and axial direction. The MOC equations are solved for each radial slice of the reactor and are coupled by a nodal diffusion solver in the axial direction. Other methods have been developed which model the axial direction using a one dimensional SN solver.

\section{Method of Characteristics}

The Method of Characteristics is a solution to the transport equation in which rays are drawn across the global geometry in discrete angles and the transport equation is integrated along those rays.

\section{Governing Equations}

The MOC equations begin with the Boltzmann transport equation with isotropic scattering.

$$
\begin{aligned}
\Omega \cdot \nabla \psi(x, \Omega, E) & +\Sigma_{t}(\boldsymbol{x}, E) \psi(\boldsymbol{x}, \Omega, E) \\
= & \frac{\chi(E)}{4 \pi k} \int_{4 \pi} \int_{0}^{\infty} \nu \Sigma_{f}\left(\boldsymbol{x}, E^{\prime}\right) \psi\left(\boldsymbol{x}, \Omega^{\prime}, E^{\prime}\right) d E^{\prime} d \Omega^{\prime} \\
& +\frac{1}{4 \pi} \int_{4 \pi} \int_{0}^{\infty} \Sigma_{s_{0}}\left(\boldsymbol{x}, E^{\prime} \rightarrow E\right) \psi\left(\boldsymbol{x}, \Omega^{\prime}, E^{\prime}\right) d E^{\prime} d \Omega^{\prime}
\end{aligned}
$$

Or

$$
\boldsymbol{\Omega} \cdot \boldsymbol{\nabla} \psi(\boldsymbol{x}, \boldsymbol{\Omega}, E)+\Sigma_{t}(\boldsymbol{x}, E) \psi(\boldsymbol{x}, \boldsymbol{\Omega}, E)=\frac{Q(\boldsymbol{x}, E)}{4 \pi}
$$


where $\mathrm{Q}$ is the isotropic fission and scattering source for position $\mathrm{x}$ and energy $\mathrm{E}$. In order to obtain a solution along the ray, the angular coordinate system is recast in the direction of the ray.

$$
\begin{gathered}
\psi(x, \Omega, E)=\psi\left(x_{m, 0}+s \Omega_{m}, E\right) \\
\Omega \cdot \nabla \psi(x, \Omega, E)=\frac{d}{d s} \psi\left(x_{m, 0}+s \Omega_{m}, E\right)
\end{gathered}
$$

Using this definition the transport can be written in direction $\mathrm{m}$ as follows.

$$
\begin{aligned}
\frac{d}{d s} \psi\left(x_{m, 0}+s \Omega_{m}, E\right)+\Sigma_{t}\left(x_{m, 0}+s \Omega_{m}, E\right) \psi\left(x_{m, 0}\right. & \left.+s \Omega_{m}, E\right) \\
& =\frac{Q\left(x_{m, 0}+s \Omega_{m}, E\right)}{4 \pi}
\end{aligned}
$$

The ordinary differential equation (2.32) has a solution which is shown below.

$$
\begin{aligned}
& \psi\left(\boldsymbol{x}_{m, 0}+s \Omega_{m}, E\right)=\psi\left(\boldsymbol{x}_{m, 0}, E\right) \exp \left(-\int_{0}^{s} \Sigma_{t}\left(\boldsymbol{x}_{m, 0}+s^{\prime} \Omega_{m}, E\right) d s^{\prime}\right) \\
& +\frac{1}{4 \pi} \int_{0}^{s} Q\left(\boldsymbol{x}_{m, 0}+s^{\prime} \Omega_{m}, E\right) \exp \left(-\int_{s^{\prime}}^{s} \Sigma_{t}\left(\boldsymbol{x}_{m, 0}+s^{\prime \prime} \Omega_{m}, E\right) d s^{\prime \prime}\right) d s^{\prime}
\end{aligned}
$$

Thus far the integral along a single ray with an arbitrary angle has been discussed. In reality the solution is solved for a discrete number of angles. The angle is discretized using a product quadrature set, meaning that the polar and azimuthal angles are chosen separately and then combined. The azimuthal angles are chosen so that $4 \mathrm{M}$ angles are represented between 0 and $2 \pi$ and the polar angles are chosen such that $2 \mathrm{~L}$ angles between -1 and 1 . Weights are assigned to each angle such that the following relationship holds.

$$
\int_{4 \pi} \psi(\boldsymbol{x}, \Omega, E) d \Omega \approx \sum_{l=1}^{2 L} \omega_{l} \sum_{m=1}^{4 M} \omega_{m} \psi_{m, l}(\boldsymbol{x}, E)
$$

Equation (2.33) is an exact representation of the transport equation along a line in direction $\Omega_{\mathrm{m}, 1}$. Two major assumptions will now be applied to this equation in order to obtain the final form. The first assumption is that the domain is two dimensional and infinite in the axial direction. This simplification can allow the rays to be based in a plane with a correction for the azimuthal dependence of the flux. 


$$
\begin{gathered}
\psi\left(\boldsymbol{x}_{m, 0}+s \Omega_{m}, \varphi_{l}, E\right)=\psi\left(\boldsymbol{x}_{m, 0}, \varphi_{l}, E\right) \exp \left(-\frac{\int_{0}^{s} \Sigma_{t}\left(\boldsymbol{x}_{m, 0}+s^{\prime} \Omega_{m}, E\right) d s^{\prime}}{\sin \varphi_{l}}\right) \\
+\frac{1}{4 \pi} \int_{0}^{s} Q\left(\boldsymbol{x}_{m, 0}+s^{\prime} \Omega_{m}, E\right) \exp \left(-\frac{\int_{s^{\prime}}^{s} \Sigma_{t}\left(\boldsymbol{x}_{m, 0}+s^{\prime \prime} \Omega_{m}, E\right) d s^{\prime \prime}}{\sin \varphi_{l}}\right) d s^{\prime}
\end{gathered}
$$

where $\varphi_{1}$ is the polar angle.

The second assumption is that the ray is traced across a discrete geometry where inside a computational node, the cross-sections and the neutron source are constant. This assumption allows the integral over the entire ray to be broken up into segments where the outgoing angular flux is an algebraic expression instead of an integral expression.

$$
\begin{aligned}
& \psi\left(\boldsymbol{x}_{m, r, 0}+s \Omega_{m}, \varphi_{l}, E\right)=\psi\left(\boldsymbol{x}_{m, r, 0}, \varphi_{l}, E\right) \exp \left(-\frac{\Sigma_{t, r}(E) s}{\sin \varphi_{l}}\right) \\
&+\frac{Q_{R}(E)}{4 \pi \Sigma_{t, r}(E)}\left(1-\exp \left(-\frac{\Sigma_{t, r}(E) s}{\sin \varphi_{l}}\right)\right)
\end{aligned}
$$

$\mathrm{r}$ denotes a specific ray inside a at source, at cross-section node $\mathrm{R}$. The incoming flux is determined by the boundary condition for nodes on the boundary or the outgoing angular flux from the previous region which is determined by evaluating equation

(2.36) at the length of cell r, sm;r.

$$
\begin{aligned}
& \psi_{m, l, r}^{\text {out }}(, E)=\psi\left(\boldsymbol{x}_{m, r, 0}+s_{m, r} \Omega_{m}, \varphi_{l}, E\right) \\
& \quad=\psi_{m, l, r}^{\text {in }}(E) \exp \left(-\frac{\Sigma_{t, r}(E) s_{m, r}}{\sin \varphi_{l}}\right)+\frac{Q_{R}(E)}{4 \pi \Sigma_{t, r}(E)}\left(1-\exp \left(-\frac{\Sigma_{t, r}(E) s_{m, r}}{\sin \varphi_{l}}\right)\right)
\end{aligned}
$$

The average angular flux along a ray segment can be found by integrating equation (2.36) from 0 to sm,r, the length of the cell, and dividing by the length of the cell. 


$$
\begin{gathered}
\bar{\psi}_{m, l, r}(\varphi, E)=\frac{\int_{0}^{s_{m, r}} \psi\left(\boldsymbol{x}_{m, r, 0}+s^{\prime} \Omega_{m}, \varphi_{l}, E\right) d s^{\prime}}{s_{m, r}} \\
\bar{\psi}_{m, l, r}(E)=\frac{\psi_{m, l, r}^{\text {in }}(E)-\psi_{m, l, r}^{\text {out }}(E)}{\frac{\Sigma_{t, r}(E) s_{m, r}}{\sin \varphi_{l}}}+\frac{Q_{R}(E)}{4 \pi \Sigma_{t, r}(E)}
\end{gathered}
$$

So far one a single ray has been considered but in reality, several parallel rays are traced across the domain. The rays are laid out across the domain in each azimuthal direction with spacing $\Delta \mathrm{m}$. In order to obtain the average angular flux in a region $\mathrm{R}$, the average flux of each ray is weighted by the volume that ray occupies.

$$
\bar{\psi}_{m, l, R}(E)=\frac{\sum_{r \in R} \bar{\psi}_{m, l, r}(E) \Delta_{m} s_{m, r}}{\sum_{r \in R} \Delta_{m} s_{m, r}}
$$

The node average angular flux is used to calculate the node average scalar flux by using the angular weights described in equation (2.34).

$$
\bar{\phi}_{R}(E)=2 \sum_{l=1}^{L} \omega_{l} \sum_{m=1}^{4 M} \omega_{m} \bar{\psi}_{m, l, R}(E)
$$

Since the problem is two dimensional, only half of the polar angles need to be simulated and the weight is modified to account for the symmetry. The final step is to apply the multigroup method.

$$
\bar{\psi}_{g, m, l, r}=\frac{\psi_{g, m, l, r}^{\text {in }}-\psi_{g, m, l, r}^{\text {out }}}{\frac{\Sigma_{t, g, r} s_{m, r}}{\sin \varphi_{l}}}+\frac{Q_{g, R}}{4 \pi \Sigma_{t, g, r}}
$$




$$
\begin{gathered}
\bar{\psi}_{g, m, l, R}=\frac{\sum_{r \in R} \bar{\psi}_{g, m, l, r} \Delta_{m} s_{m, r}}{\sum_{r \in R} \Delta_{m} s_{m, r}} \\
\bar{\phi}_{g, R}=2 \sum_{l=1}^{L} \omega_{l} \sum_{m=1}^{4 M} \omega_{m} \bar{\psi}_{g, m, l, R}
\end{gathered}
$$

The last remaining issue is to use the scalar flux in each group and region to determine the neutron source $Q_{g, R}$.

$$
Q_{g, R}=\sum_{g^{\prime}=1}^{G} \Sigma_{s_{0}, R, g^{\prime} \rightarrow g} \phi_{g^{\prime}, R}+\frac{\chi_{g, R}}{k} \sum_{g^{\prime}=1}^{G} \nu \Sigma_{f, g^{\prime}, R} \phi_{g^{\prime}, R}
$$

\section{Ray Tracing}

So far we have assumed that the lengths of the rays, sm,r, are known. Generation of all the ray segments for the whole core geometry requires enormous computational memory and programming effort. To avoid this problem, the ray segments are generated only for each cell type, and the rays defined for each cell are linked to the rays of the adjacent cells through path linking. For path linking, each ray must align itself exactly with its reflective counterpart at the cell boundary. To meet this condition, the ray spacing and azimuthal angle are adjusted from the evenly spaced initial angles and uniform ray spacing determined by the input parameters. 


$$
\begin{gathered}
N_{x}^{m}=\operatorname{ceil}\left(\frac{P}{\Delta_{m_{0}}}\left|\sin \left(\alpha_{m_{0}}\right)\right|\right) \\
N_{y}^{m}=\operatorname{ceil}\left(\frac{P}{\Delta_{m_{0}}}\left|\cos \left(\alpha_{m_{0}}\right)\right|\right) \\
\Delta_{m}=\frac{P}{\sqrt{\left(N_{x}^{m}\right)^{2}+\left(N_{y}^{m}\right)^{2}}} \\
\tan \left(\alpha_{m}\right)=\frac{N_{x}^{m}}{N_{y}^{m}}
\end{gathered}
$$

For PWR cores, the pin is normally the smallest repeated structure and is used as the cell. In a BWR the assembly is the smallest repeated structure and is used as the cell. Since only PWR cores are considered in this work, the pin is always used as the cell.

The pin cell is broken into 8 azimuthal segments and the user defines an arbitrary number of concentric circles located at the center of the pin cell. Each pin type is traced for each modular ray and direction to obtain the lengths inside the pin. The link between the incoming and outgoing ray is also stored in order to easily construct the path linking between neighboring pins. Once each pin type has been traced, the global rays are constructed using path linking. Instead of storing all of the structure inside a global ray, only the local pin index and the local ray index are stored for each part of the ray, greatly reducing the global storage requirements. As the global rays are traced in the MOC subroutine, the local ray information is recovered.

Another inherent problem with discrete rays is that the volume that the rays represent is not equivalent to the cell volume. Each ray represents a beam of neutrons that is rectangular. In curve-linear geometry, these rectangles do not accurately match the true geometry. Therefore the ray lengths are adjusted to conserve the total volume of each region. This is done by simply adjusting the lengths, sm, $\mathrm{r}$ to preserve the total volume in region $\mathrm{R}$. 


$$
s_{m, r}^{\text {corr }}=\frac{s_{m, r} V_{R}}{\sum_{r \in R} s_{m, r} \Delta_{m}}
$$

The corrected lengths are used for both the MOC ray sweeping routine and the calculation of the region average angular flux.

\section{Source Iteration}

The source iteration for the MOC calculation is slightly different than the diffusion equation because the scattering source is dependent on the angular flux from other angles. The number of free variables in MOC makes it difficult to set up into a matrix form. Two iteration loops are used in order to converge the scattering source and the fission source. The outer most loop is the typical fission source iteration power method described for the FMFD case. Since the equations are not in matrix form, the application of Wielandt shift acceleration is not practical. An inner iteration loop is performed to converge the scattering source with a fixed fission source. It should be noted that the power method requires that the fission source does not change during a power iteration. Inside the inner loop, all rays inside each angle are swept from beginning to end. To minimize memory access, the ray is also swept in the reverse direction in the inner most loop.

\section{d. Stochastic Methods}

The Monte Carlo method is a stochastic method for solving the transport equation by simulating individual neutrons and computing a solution based on statistical averages. The Monte Carlo method is advantageous because there is no discretization error introduced in space, energy, or angle. Therefore the error encountered in the solution is purely statistical. The neutrons are simulated from their birth into the system and pseudo-random numbers are used to sample probability distribution functions to determine if, a reaction occurs, the type of reaction, and the secondary energy and angle of the particle. This process is repeated for millions of neutrons in the system.

The statistical error in these calculations follows the Central Limit Theorem if $\mathrm{N} \gg 1$.

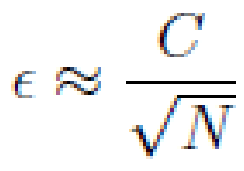

Therefore the statistical error decreases in inverse proportion, "inversely proportional", to the square root of the number of particles simulated. 


\section{Coarse Mesh Finite Difference Acceleration}

The Coarse Mesh Finite Difference method is an acceleration technique that takes advantage of effciency of the power method with Wielandt shift in the diffusion solver.

CMFD uses the standard definition of node balance from the FMFD solution but a nonlinear correction factor is added to the current term at each nodes surface to account for the deficiencies of the diffusion method.

$$
J_{e}=\frac{2 D_{P} D_{E}\left(f_{P, e} \phi_{P}-f_{E, w} \phi_{E}\right)}{D_{E} f_{P, e} h_{P}+D_{P} f_{E, w} h_{E}}+\hat{D}_{e}\left(\phi_{P}+\phi_{E}\right)
$$

The nonlinear correction factor $\widehat{D}$ is defined using the surface current from the MOC calculation.

$$
\hat{D}_{e}=\frac{J_{e}^{M O C}-\frac{2 D_{P} D_{E}\left(f_{p_{, e}} \phi_{P}-f_{E, w} \phi_{E}\right)}{D_{E} f_{P, e} h_{P}+D_{P} f_{E, w} h_{E}}}{\phi_{P}+\phi_{E}}
$$

The goal of the CMFD system is to limit the number of times the MOC sweep algorithm is called. To do this efficiently several layers of CMFD can be used in series that target different aspects of the global solution. The layers of the iteration scheme are broken down into three main levels: few group CMFD, multigroup CMFD, and MOC sweeping.

\section{e. CMFD Acceleration}

The few group CMFD is the lowest level of the acceleration method. The goal of this level is to contain the entire system into one matrix. In this work two group pin wise regions are selected as the computational grid. Two groups are sufficient to accurately couple the thermal and fast energy groups and allow for a quick and efficient solution of the global system.

When various layers are used, it is useful to limit the implementation of the power method to a single level of the method. The power method is implemented to the few group CMFD level because it allows for Wielandt shift to be implemented. The few group CMFD level determines the eigenvalue of the system and the spatial distribution of the fission source. The remaining levels use the fission source defined by the few group CMFD. 
The multigroup CMFD solver uses the same number of energy groups as the MOC solution and pin wise spatial regions. Because the number of energy groups can be very large, it is impractical to store the entire multigroup CMFD system into a single matrix. Therefore the multigroup CMFD method will be solved group wise starting from the highest energy group and sweeping downward and a second iteration is performed over all groups that have upscattering sources.

\section{CMFD Iteration Scheme}

The global CMFD iteration scheme is based on a V-cycle iteration. The few group CMFD first solves for the eigenvalue with cross-sections homogenized based on a uniform global flux and the nonlinear correction factors are assumed to be zero. This gives a very good initial condition for the fission source. Then the multigroup CMFD system is solved to resolve the global spectral effects. This is followed by the first MOC sweep. Once the MOC sweep is complete, the pin average cross-sections, fluxes, and surface currents are determined and nonlinear correction factors are determined for the multigroup CMFD. The multigroup CMFD is solved again and the solution is reduced to the few group CMFD. This method is repeated until the fission source stops changing, the eigenvalue is converged, and the residual in the multigroup residual is less than a given tolerance.

\subsection{Implementaion of Multiscale Neutronics Method}

\section{Boundary Response Function}

The interface between level 1 and level 1-1 requires a method to determine the boundary conditions for the local solution. The boundary response function relates the incoming angular flux to the outgoing angular flux. In this analysis the local domain is considered convex so that neutrons leaving the local system must interact with the surrounding medium to return into the local domain. The assumption of domain convexity is consistent with practical problems that will be solved in this work.

$$
\begin{aligned}
& \psi(x, \Omega, E)= \\
& \int_{0}^{\infty} \int_{\Omega \cdot n<0} \int_{\delta R} A\left(k_{e f f}, x^{\prime} \rightarrow \boldsymbol{x}, \Omega^{\prime} \rightarrow \Omega, E^{\prime} \rightarrow E\right) \psi\left(\boldsymbol{x}^{\prime}, \Omega^{\prime}, E^{\prime}\right) d \boldsymbol{x}^{\prime} d \Omega^{\prime} d E^{\prime} \\
& x \in \delta R, \quad \Omega \cdot \boldsymbol{n}>0, \quad 0<E<\infty
\end{aligned}
$$

A is the boundary response function which describes the relationship between the outgoing angular flux and the incoming angular flux at all points on the surface $\delta \mathrm{R}$, all energies $\mathrm{E}$, and angle. If the boundary response function is known the reduced domain transport problem can be 
run without approximation. Unfortunately, the computational demand required to calculate the response function is significantly greater than calculating the full domain with the transport solver. Approximate response functions are constructed to preserve the level 1-1 solution but also model as much physics as possible. Various levels of approximation are introduced in this work with increasing levels of complexity.

The simplest method for approximating the boundary function is to preserve the level 1-1 solution but assume the distribution is at across the level 1-1 mesh. In space the response function is simply a delta function on the surface. A neutron leaving the local boundary is assumed to reenter at the identical point in space. In angle the incoming angular flux is assume to be isotropic but the net current across the surface is preserved, which is equivalent to the double P0 or DP0 assumption. In energy the multigroup global solution is assumed to be a histogram in energy. Since the multigroup angular flux is an integral parameter in energy, the magnitude of the angular flux must be divided by the width of the multigroup bin. Using these three approximations, the local solution boundary equation can be written as follows.

$$
\psi(\boldsymbol{x}, \Omega, E) \approx \frac{\phi_{g}(\boldsymbol{x})+2 \boldsymbol{n} \cdot \boldsymbol{J}_{g}(\boldsymbol{x})}{4 \pi\left(E_{g-1}-E_{g}\right)} \quad \boldsymbol{x} \in \delta R, \quad \boldsymbol{\Omega} \cdot \boldsymbol{n}>0, \quad E_{g}<E<E_{g-1}
$$

If the local solver is also a multigroup method with $\mathrm{H}$ energy groups, the response function can be written as follows.

$$
\psi_{h}(\boldsymbol{x}, \Omega)=\frac{\phi_{g}(\boldsymbol{x})+2 \boldsymbol{n} \cdot \boldsymbol{J}_{g}(\boldsymbol{x})}{4 \pi} \frac{E_{h-1}-E_{h}}{E_{g-1}-E_{g}} \quad \boldsymbol{x} \in \delta R, \quad \Omega \cdot \boldsymbol{n}>0, \quad E_{h} \in E_{g}
$$

This equation assumes that the coarse energy group structure is aligned with the fine energy group structure. The remaining approximations discussed here focus on improving the three variable; space, angle, and energy. The approximations in each variable can generally be paired together to generate a response function that has the desired level of detail.

An improvement to the DP0 approximation in angle is to use the P1 approximation where the angular flux is a linear function of angle. This is the natural form of the diffusion equation which is likely to be the global solver. The form of the response equation is very similar to the DPO but is also a function of incoming angle.

$$
\psi(\boldsymbol{x}, \Omega, E) \approx \frac{\phi_{g}(\boldsymbol{x})+3 \Omega \cdot \boldsymbol{J}_{g}(\boldsymbol{x})}{4 \pi\left(E_{g-1}-E_{g}\right)} \quad \boldsymbol{x} \in \delta R, \quad \boldsymbol{\Omega} \cdot \boldsymbol{n}>0, \quad E_{g}<E<E_{g-1}
$$


Another improvement that can be made is to use the lattice calculation to determine predefined angular shape factors which describe the ratio of the incoming angular flux and the partial incoming current. Although this method gives a much more accurate description of the angular flux shape, it also requires a significant amount of data to be saved in the lattice calculation.

$$
\psi(\boldsymbol{x}, \boldsymbol{\Omega}, E) \approx \frac{\phi_{g}(\boldsymbol{x})+2 \boldsymbol{n} \cdot \boldsymbol{J}_{g}(\boldsymbol{x})}{4 \pi\left(E_{g-1}-E_{g}\right)} \frac{f(\boldsymbol{\Omega} \cdot \boldsymbol{n})}{2} \quad \boldsymbol{x} \in \delta R, \quad \boldsymbol{\Omega} \cdot \boldsymbol{n}>0, \quad E_{g}<E<E_{g-1}
$$

So far the response function has not been a function of the outgoing angular flux. In the next method, the shape of the incoming flux is influenced by the outgoing flux. The simplest method is to reflect the outgoing flux back into the domain and scale the magnitude to preserve the partial currents obtained from the global solution.

$$
\begin{aligned}
& \psi(\boldsymbol{x}, \boldsymbol{\Omega}, E)=\frac{\phi_{g}(\boldsymbol{x})+2 \boldsymbol{n} \cdot \boldsymbol{J}_{g}(\boldsymbol{x})}{\phi_{g}(\boldsymbol{x})-2 \boldsymbol{n} \cdot \boldsymbol{J}_{g}(\boldsymbol{x})} \frac{\psi_{g}\left(\boldsymbol{x}, \boldsymbol{\Omega}^{\prime}\right)}{E_{g-1}-E_{g}} \\
& \boldsymbol{x} \in \delta R, \quad \Omega \cdot \boldsymbol{n}>0, \quad E_{g}<E<E_{g-1} \\
& \boldsymbol{\Omega}^{\prime}=\boldsymbol{\Omega}-2 \boldsymbol{n}(\boldsymbol{\Omega} \cdot \boldsymbol{n})
\end{aligned}
$$

The energy variable is much more difficult to handle for two reasons. The first is because the general multigroup formulation is only a zeroth order method so no high order moments exist to allow for reconstruction of the energy grid. The second reason is because of the tight coupling between energy groups. A good boundary response function must be able to account for fast neutrons leaking out of the local domain to return with lower energies as well as thermal neutrons leaving, causing fission, and returning at higher energies. These two methods will be dealt with in separate sections. First methods to prolongate the energy variable from the few group structure to the fine group structure will be discussed. After a fine group structure is obtained the methodology is developed to allow for the transfer of neutrons between energy groups when interacting with the local system boundary.

The simplest method for expanding the few group global solution to a fine group local boundary condition is to use a shaping function, $f g(E)$, to reconstruct the energy variable. This shaping function can be defined using an analytic shape or calculated from the lattice calculation. 


$$
\begin{aligned}
\psi(\boldsymbol{x}, \Omega, E)=\frac{\phi_{g}(\boldsymbol{x})+2 \boldsymbol{n} \cdot \boldsymbol{J}_{g}(\boldsymbol{x})}{4 \pi} f_{g}(E) & \\
x & \in \delta R, \quad \Omega \cdot \boldsymbol{n}>0, \quad E_{g}<E<E_{g-1}
\end{aligned}
$$

The shape function can be used to describe the original method of assuming neutrons were evenly distributed in energy.

$$
f_{g}(E)=\frac{1}{E_{g-1}-E_{g}}
$$

The form of the multigroup shape function can be written as a discrete function coupling few group energy $g$ to the fine group energy $h$.

$$
\psi_{h}(\boldsymbol{x}, \Omega)=\frac{\phi_{g}(\boldsymbol{x})+2 \boldsymbol{n} \cdot \boldsymbol{J}_{g}(\boldsymbol{x})}{4 \pi} f_{g \rightarrow h} \quad \boldsymbol{x} \in \delta R, \quad \boldsymbol{\Omega} \cdot \boldsymbol{n}>0, \quad E_{h} \in E_{g}
$$

Another method for the shape parameter is to use the outgoing angular flux to define the shaping function. There is no physical reason for outgoing and incoming angular flux to have the same energy shape but this approximation should provide sufficiently accurate results if the flux is fairly asymptotic.

$$
f_{g}(E)=\frac{\int_{\Omega \cdot n>0}(\Omega \cdot n) \psi(\boldsymbol{x}, \Omega, E) d \Omega}{\int_{E_{g}}^{E_{g-1}} \int_{\Omega \cdot n>0}(\Omega \cdot \boldsymbol{n}) \psi(\boldsymbol{x}, \Omega, E) d \Omega d E}
$$

The final method considered is to solve an extra set of fixed source equations to calculate flux moments in energy. One method for calculating these moments is the Discrete Generalized Multigroup Method [13, 12, 11]. Forget has proposed a mixed energy calculation in which energy moments are tracked in a region surrounding the

multigroup region. These energy moments allow for the fine energy flux to be reconstructed on the boundary of the fine/coarse energy interface without needing to assume a shape for prolongation. In this case the approximation is made at the interface where the moments are chosen to be tracked. The region where the moments are tracked provides an identical low level solution but also provides a buffer region to reconstruct the fine energy group fluxes. 
Now that methods have been developed to determine the fine group energy structure at the interface, methods to describe the transfer of neutrons between energy groups outside of the local domain are discussed. The simplest method is to not assume any transfer between energy groups. Since the currents at the surface have already been determined this method simply enforces that condition.

Developing a method that allows the outgoing neutrons to change energy when returning to the system is a much more difficult problem. Since the net incoming and outgoing flow of neutrons across the local domain boundary are known, the remaining unknown is a function that relates them. The construction of a $\mathrm{G} 2$ response function from $2 \mathrm{G}$ inputs, where $\mathrm{G}$ is the number of energy groups, requires additional information.

The additional information in this case comes from the source term in the neighboring assembly. The source term describes how neutrons appear in the region close to the assembly. Since neutrons do not move very far from the boundary before interacting, the source term of the adjacent assembly is a reasonable approach.

\section{Space}

The spatial variable is also difficult to accurately quantify. When neutrons leave the local system, they move through the surrounding medium and can reenter the local system. The distance a neutron travels is directly proportional to the mean free path of a neutron in the system. In the reactor systems considered in this work, the mean free path of a neutron is on the order of $2-3 \mathrm{~cm}$. This suggests that the average neutron will only move a few pins away from the point where the neutron leaves the local system. High energy neutrons do have mean free paths that are significantly longer than the average mean free path but it is difficult to determine where on the surface these neutrons will interact, if they ever do.

The approach taken so far in this chapter is that the spatial response is treated as a delta function. This means that a neutron reenters the local system at the exact point it leaves. Although there are some limitations to this assumption it provides sufficient accuracy and is the only method used in this work.

A few methods have been considered to treat the spatial coupling and will briefly be discussed here. The first method is to pretabulate a spatial response function on a limited domain. Since the neutrons are not expected to travel very far after leaving the limited domain, a response function could be introduced to distribute the neutrons across the pins close to the exiting location. The extent of this local response would be determined by the ability of surrounding medium to transport a neutron away from the point at which it leaves the local domain. A second method to determine the spatial coupling is to use the global solution to 
calculate a series of fixed source problems to determine the spatial response function. Since the global solution can be solved efficiently, this method is not expected to greatly increase the computational burden.

\section{Post-Refinement (OneWay Coupling) Method}

Post-Refinement methods are one way coupling methods from the nodal diffusion solver back to the higher order transport solver. These methods impose the core level solution by assigning either an albedo type boundary condition or a fixed boundary source problem. These boundary assignments have a fundamentally different solution technique which must be applied. The albedo boundary condition provides the ratio of the incoming angular flux to the outgoing angular flux. With these boundary conditions the problem becomes an eigenvalue problem and a new eigenvalue is determined which represents the scaling of the fission source for the local domain. The fixed boundary source problem is not an eigenvalue problem. In this method the angular flux at the boundary is specified and the fission source is scaled with the eigenvalue from the global calculation. Both methods have advantages and disadvantages in the solution technique and implementation.

The solution procedure for the post-refinement method begins with a full core diffusion calculation. Once the diffusion calculation has converged to the given tolerance, the surface fluxes and net currents are extracted at the boundary of the multiscale region. In the case of the albedo method, the ratio of the incoming and outgoing partial currents are used for all angles, and are assigned to all fine groups which the coarse group solution represents. For the incoming angular flux, the surface flux and net current are used to project angular and energy shapes using one of the expansion methods mentioned above. Finally the local solution is solved and the pin power shape is normalized and projected onto the global solution. There are no changes made to the global solution except the shape of the pin power distribution.

The projection of the pin powers onto the global solution is one of the main shortfalls of the post-refinement method because it is completely dependent on the global solution. For example, if the assembly power is off by two percent, then the pin powers in that assembly will also be off by two percent. The post-refinement method does have some benefits as well. First of all, the post-refinement method could be implemented into any code system with only minor modifications. The second is the post-refinement method can allow the user to specify a buffer region where the local solution may not be accurate. The buffer region only serves to decrease the impact of the boundary condition on the solution. When the pin power projection is determined only the solution outside the buffer region is used. 


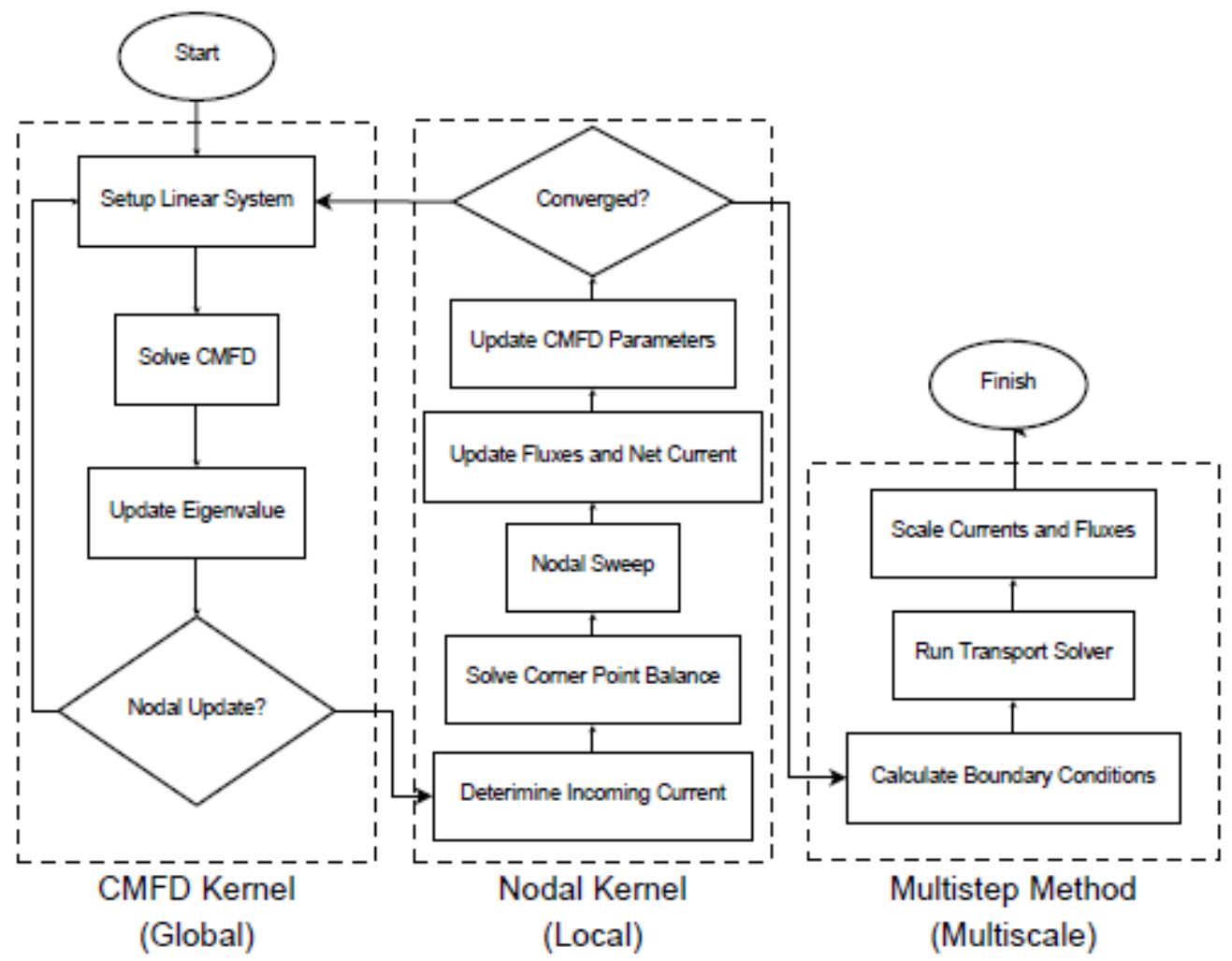

\section{Post-Refinement Fixed Boundary Source Method Implementation}

The post-refinement methods use the global CMFD solver to fully converge the global FMFD linear system. The net current and surface flux from the global solution are calculated on the boundary of the multiscale region. The angular flux at the boundary of the multiscale region is defined using the P1 angular distribution. The energy projection is performed using a predefined flux distribution obtained from the energy shape of the surface fluxes of the lattice calculation.

During the fixed boundary source calculation the fission source is scaled by the eigenvalue calculated from the global solution. Since the fixed source problem is not an eigenvalue problem, the acceleration scheme is slightly different. The CMFD solution is rewritten to have boundary sources instead of albedo boundary conditions. Since an eigenvalue iteration does not need to be performed, the fission and scattering sources can be moved to the left hand side of the equation, leaving only the boundary source on the right hand side of the system of equations.

$$
\left(\mathbb{M}-\lambda_{\text {global }} \mathbb{F}\right) \phi=C_{0} J_{\text {in }}
$$


The matrix $\mathrm{M}$ is slightly modified to handle the fact that the boundary currents are on the source side of the equations. The nonlinear correction factors from the CMFD are still added into the migration matrix to preserve the higher order net current.

\section{Post-Refinement Albedo Boundary Condition Method Implementation}

The net current and surface flux from the global FMFD solution are combined to calculate the ratio of the incoming and outgoing partial currents.

$$
\beta=\frac{\phi+2 \boldsymbol{J}^{\text {net }} \cdot \boldsymbol{n}}{\phi-2 \boldsymbol{J}^{\text {net }} \cdot \boldsymbol{n}}
$$

The albedos are applied to the local boundary and then a local eigenvalue calculation is performed. The local eigenvalue calculation is identical to the global, except the extent of the domain is expanded. The albedo is applied uniformly for all energy groups contained in the coarse energy groups.

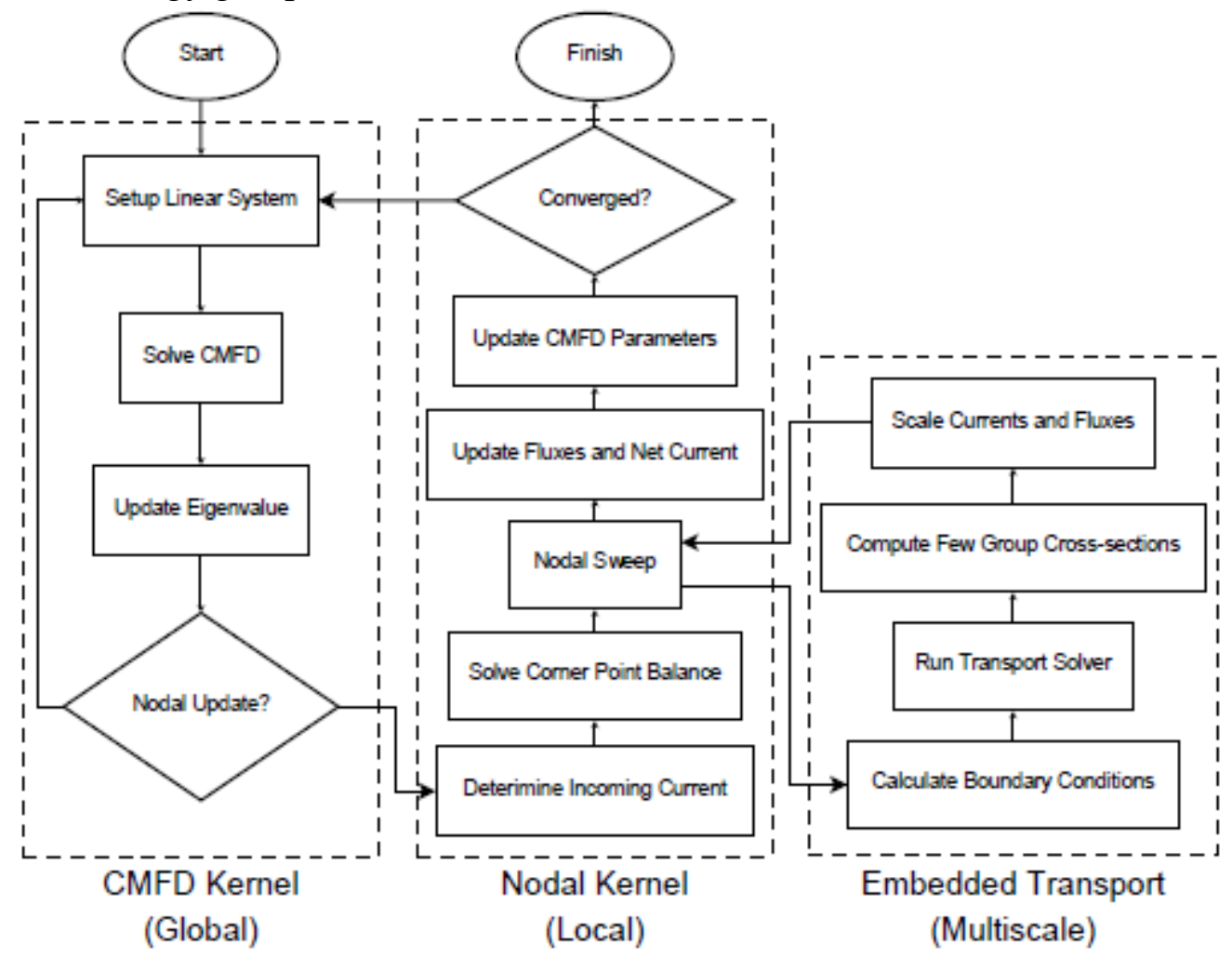




\section{Embedded Iteration Method}

The embedded iteration method couples all simulation levels together to ensure that the interface between the levels provide a consistent balance of neutrons. The local solvers are directly embedded into the global solution and neutrons are strictly preserved at the interfaces. The iteration strategy for the embedded method is shown in figure 3.2. The embedded iteration methodology can also be used to implement a more detailed transport calculation such as 3-D MOC into a global transport solution such as SN or 2D 1D MOC. The major difference would be preserving the angular flux at the interface.

\section{Embedded Iteration Method Implementation}

The embedded iteration method implemented in the research here relies on three major solvers to be interfaced together. The first is assembly-wise diffusion in which the assembly is collapsed into one homogeneous material with discontinuity factors defined on the surfaces. The second solver is a pin-wise diffusion solver in which each pin cell is collapsed into a homogeneous material with discontinuity factors defined on the surfaces of each pin cell. The third solver is the MOC which solves the transport equation with explicit geometry specifications. The diffusion based methods are capable of being solved with any energy group structure during the simulation and the MOC only uses the finest energy group structure.

The levels are defined so that level 1 always solves 2 group assembly-wise diffusion with is consistent with the standard nodal methods. Level 2 and 3 generally involve pin-wise diffusion with more energy groups. The highest level MOC solver is used to obtain the transport solution on a very localized region. Level 1 is the only level that performs the eigenvalue search because it is the only level that contains the global information. All subsequent levels are solved as a standard fixed source problem with fission sources defined by the level 1 solution.

The levels are coupled together using the CMFD framework. Similar to standard multiscale methods, the higher levels provide information to the lower levels to ensure consistency between the levels. Standard multiscale methods do this through the use of a source term but since the original problem is an eigenvalue problem, another method must be chosen.

Instead of adding a source term, nonlinear correction factors, $\widehat{D}$, are used here to preserve the neutron balance.

The integration of both the assembly-wise and pin-wise diffusion solvers into the CMFD framework is straightforward. The projection of the partial currents between level 1 and level $1+1$ is simply an energy prolongation. For these energy prolongation operators, the outgoing partial current spectrum is used, which can create problems if the interfaces are not chosen carefully. However, in most cases and in the work here this is a reasonable assumption. 
The interface between a diffusion solver and the MOC solver is more difficult because the differences between diffusion theory and transport must be defined consistently.

In order to do this correctly, a discontinuity factor must be defined on the MOC side of the interface to ensure an accurate partial current is used for the boundary condition of the interface. This discontinuity factor arises because of the differences in the definition of the surface flux between the two different methods. In diffusion theory, the surface flux is directly related to the two partial currents on the interface but in transport theory, these two quantities are not algebraically related.

$$
\phi_{s, \text { diff }}=2\left(J^{+}+J^{-}\right)
$$

The difference in the definitions of the surface fluxes must be taken into account to ensure the net current is calculated consistently at the interface.

$$
f_{M O C}=\frac{\phi_{s, M O C}}{2\left(J_{M O C}^{+}+J_{M O C}^{-}\right)}
$$

Since the MOC discontinuity factor is not known before the calculation begins, it is iteratively obtained during the solution. Then the incoming partial current can be calculated with the MOC discontinuity factor.

$$
J_{M O C}^{i n}=\frac{\phi_{s}}{4 f_{M O C}} \pm \frac{J^{n e t}}{2}
$$

Once the incoming partial current is obtained, they can be projected in energy and angle. The energy projection uses the outgoing partial current spectrum and the angle projection uses a predefined shape from the lattice calculation. The last step required to complete the embedded iteration is to restrict the solution from level 1 to level 1-1. The cross-sections are collapsed using the standard method depending on the reaction type. Nonlinear correction factors are defined on the surface of each of each pin to preserve the net current from level 1 in most cases. However, the interface again needs special treatment to ensure a smoothly converging solution. In order to obtain the net current at the interface, the response of the level 1-1 solver to the level 1 outgoing partial current must be determined. This response is obtained by doing a single node solve. A single node is constructed with two boundary conditions: the incoming partial current on the 
interface and the average node flux from the previous iteration. The FMFD method provides an algebraic relationship between these two boundary conditions.

$$
\begin{gathered}
J_{\text {diff }}^{\text {in }}=\frac{\phi_{s}}{4 f_{\text {dif }}} \pm \frac{J^{\text {net }}}{2} \\
J_{\text {diff }}^{\text {out }}=\frac{2 D \phi-(4 D-h) J_{\text {diff }}^{\text {in }}}{(4 D+h)} \\
J^{\text {net }}= \pm\left(J_{\text {diff }}^{\text {in }}-J_{\text {diff }}^{\text {out }}\right) \\
\phi_{s}=2\left(J_{\text {diff }}^{\text {in }}+J_{\text {diff }}^{\text {out }}\right) f_{\text {diff }}
\end{gathered}
$$

Once the partial currents are determined, the net current and surface flux can be calculated and the nonlinear correction factors can be determined for the interface. The iteration strategy used is the standard "V-cycle". Level 1 is solved first to obtain the global fission source and eigenvalue. The levels are increased step by step until the maximum level is reached. The levels are then decreased back to 1 . This process is repeated until the global fission source norm and the eigenvalue both reach their convergence tolerance.

\section{Assessment of the Post-Refinement Multiscale Method}

In order to test the accuracy and speed-up of the post-refinement multiscale method, a series of one dimensional test problems are developed to determine the performance of these methods in various geometric regions. First an all (Low Enriched Uranium) LEU core will be analyzed then two (Mixed Oxide / Low Enriched Uranium) MOX/LEU cores will be examined to see how the method works with a more heterogeneous flux shape.

\section{Figures of Merit for Analysis}

Six figures of merit are chosen to determine the effectiveness of the multiscale method. The first figure of merit is the speedup factor. This is the the ratio of the time to perform the full core transport solution divided by the time to run the multiscale solution. 


$$
\tau=\frac{t^{\mathrm{MOC}}}{t^{\mathrm{MS}}}
$$

The next figure of merit compares the eigenvalue calculated in the solution. For the fixed boundary source method, the eigenvalue is identical to the global diffusion eigenvalue but when the albedo boundary conditions are specified, a new eigenvalue is calculated for the local region. When albedo boundary conditions are used, the local eigenvalue will be compared but in all other cases, the global eigenvalue is compared.

The traditional way to display eigenvalue differences for nuclear reactor problems is to show the difference in percent milli-k ( $\mathrm{pcm})$.

$$
\Delta \mathrm{k}=\left(\mathrm{k}^{\mathrm{MOC}}-\mathrm{k}^{\mathrm{MS}}\right) \times 10^{5}
$$

The next four figures of merit deal with the accuracy of the local and global solution. The first FOM is the error in the peak pin power. This is defined as the absolute difference between the maximum pin power from the MOC solution and the multiscale solution.

$$
E^{\text {peak }}=\left|\max \left(P^{\mathrm{MOC}}\right)-\max \left(P^{\mathrm{MS}}\right)\right|
$$

The second FOM is the maximum error in the pin powers. This is the maximum absolute difference in any pin in the core between the MOC solution and the multiscale solution.

$$
E^{\max }=\max \left(\left|P^{\mathrm{MOC}}-P^{\mathrm{MS}}\right|\right)
$$

The third FOM is the root mean square of the pin power error in the whole core.

$$
E^{\mathrm{RMS}}=\sqrt{\frac{\sum_{i=1}^{N_{\text {global }}}\left|P_{i}^{\mathrm{MOC}}-P_{i}^{\mathrm{MS}}\right|^{2}}{N_{\text {global }}}}
$$

The last FOM is the root mean square of the pin power shape in the peak assembly. This figure of merit was developed to ensure that multiscale method was improving the shape and the results are not due to cancellation of projection errors. 


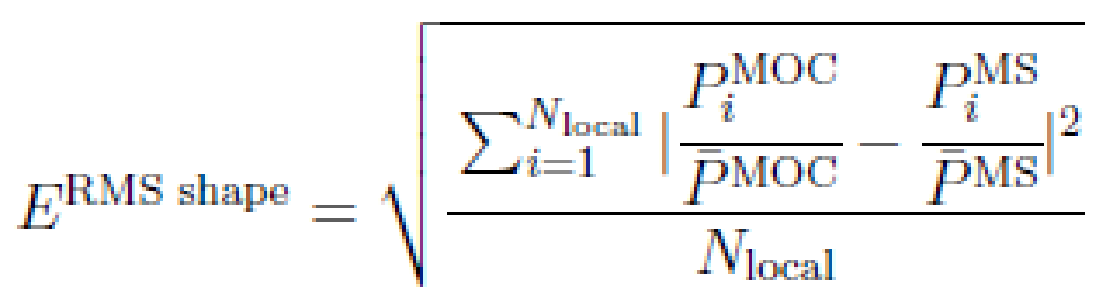

The figures of merit developed above will typically be compared as an improvement over the diffusion solution with pin power reconstruction.

$$
I=E^{\mathrm{Diff}}-E^{\mathrm{MS}}
$$

Therefore any positive value will indicate an improvement over the standard methodology and if the value is negative, the multiscale solution is worse than the diffusion solution.

\section{One Dimensional Analysis}

Three different reactor core models are developed for the purpose of testing the various methods. The assembly designs are based on the well established C5G7 benchmark [22]. The assemblies are approximated into one dimensional assemblies by preserving the pin pitch and the fuel to moderator ratio. Cross-sections provided for each material from the $\mathrm{C} 5 \mathrm{G} 7$ benchmark are used for 7 group calculations. Another set of 33 group cross-sections for the C5G7 benchmark were provided by Forget [23]. The 33 group cross-sections provide a more realistic representation of the group structure traditionally used for transport calculations.

\section{Assembly Layouts}

The assemblies shown in this work are based on 17x17 PWR fuel assemblies defined in the C5G7 benchmark. The one dimensional assembly is comprised of 16 fuel pins and a water hole which approximately preserves the ratio of water holes to fuel pins in the actual assembly. In the case where a control rod is inserted into the assembly, the water hole is filled with a control slab the same dimension as the fuel surrounded by water. Table 4.1 describes the dimensions of the pins and assembly. Two different assemblies are defined. The first is a LEU assembly. All of the pins are uniformly enriched. The geometry can be seen in Figure 4.1. The second assembly is a MOX assembly, three different pin enrichments are used to flatten the power profile of the assembly and is shown in Figure 4.2. Lastly a reflector assembly is defined as an assembly pitch in width filled with the moderator material. 
\begin{tabular}{|l|l|l|l|l|l|l|l|l|l|l|l|l|l|l|l|l|}
\hline UO2 & UO2 & UO2 & UO2 & UO2 & UO2 & UO2 & UO2 & H2O & UO2 & UO2 & UO2 & UO2 & UO2 & UO2 & UO2 & UO2 \\
\hline
\end{tabular}

Figure 4.1: LEU Assembly

\begin{tabular}{|l|l|l|l|l|l|l}
\hline 4.3 & 7.0 & 7.0 \\
\hline
\end{tabular}

\begin{tabular}{l|l|l|}
7.0 & 7.0 & 4.3 \\
\hline
\end{tabular}

Figure 4.2: MOX Assembly

LEU Core

The first core design is comprised of seven LEU assemblies with a reflector assembly on the right side. A reflective boundary condition is imposed on the left boundary and a vacuum boundary condition on the right. Figure 4.3 shows the assembly layout.

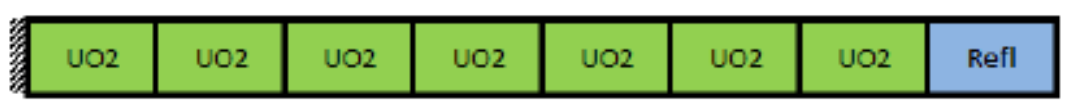

Figure 4.3: LEU Core Layout

Multiscale Regions

Three multiscale regions are run for the post-refinement cases. Figure 4.4 shows the region of interest and buffer regions.
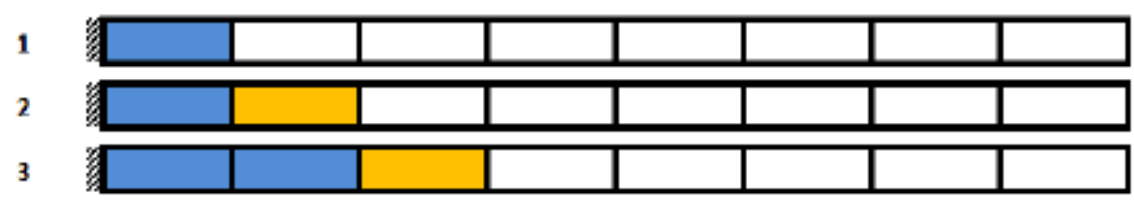

\section{Region of Interest $\quad$ Buffer}

Figure 4.4: Multiscale Regions for 1-D LEU and MOX 1 Cores

The first multiscale region is a single assembly and does not have a buffer region. The second multiscale region is two assemblies, one of which is a buffer. The last multiscale region is three assemblies, one of which is a buffer. The inclusion of two assemblies into the region of interest will help minimize the impact of assembly errors by making the region larger.

\section{Results for LEU Core}

The one dimensional LEU core is the simplest case because of the fairly homogeneous fuel loading. For this case, the current pin power reconstruction (PPR) methodology does a very good job of reconstructing the pin powers in the reactor. The error in the diffusion solution with pin power reconstruction is shown in Figure 4.5. 


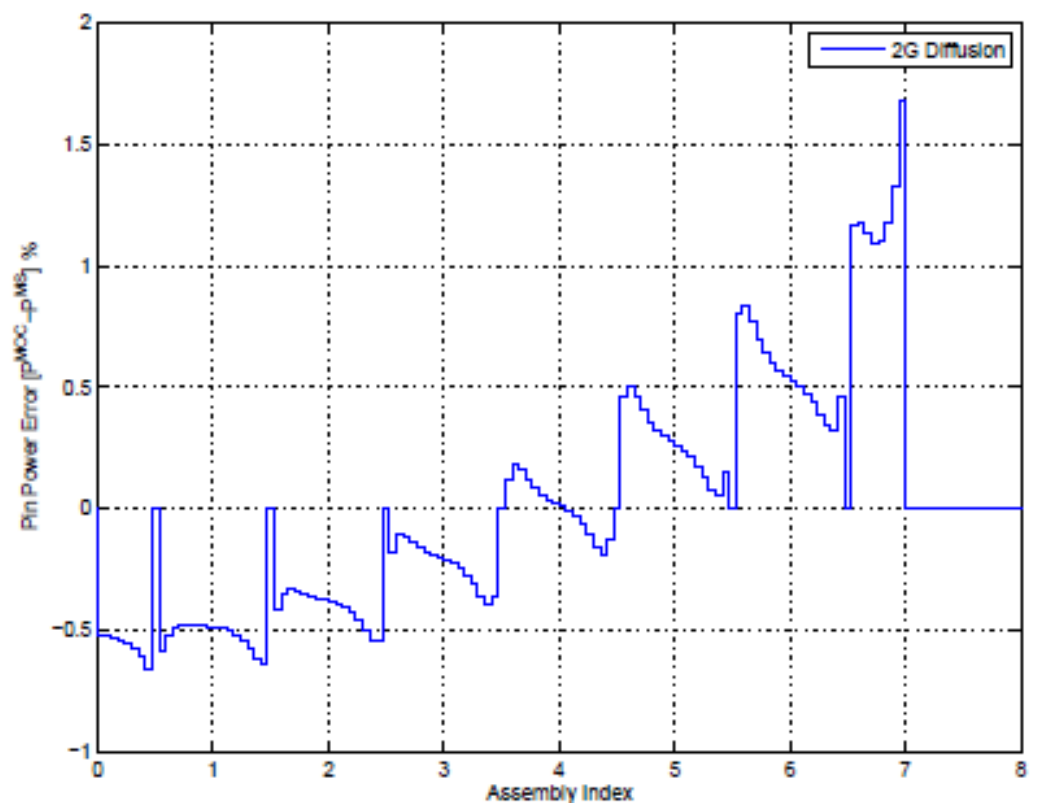

Figure 4.5: Pin Power Error in PPR for Mixed Energy LEU Core

The largest error in the LEU core occurs at the core reflector interface. Generally errors at the core reflector interface are acceptable because the peak power does not occur near the reflector. Since the diffusion solution have very little error, the multiscale method does not provide much advantage. The same energy multiscale cases all slightly increase the accuracy of the LEU core but with the mixed energy cases it is possible to make the solution worse. Even though the solutions can get worse, it is informative to examine the performance of all multiscale methods for cases where the standard methodology is not suffcient.

\section{MOX1 Core}

Two MOX cores are designed to test different behaviors. The first is designed to test the heterogeneity caused by the addition of MOX into the reactor. Four LEU and three MOX assemblies are laid out in the core and a single reflector assembly is located on the right side. Figure 4.6 shows the layout of the assemblies.

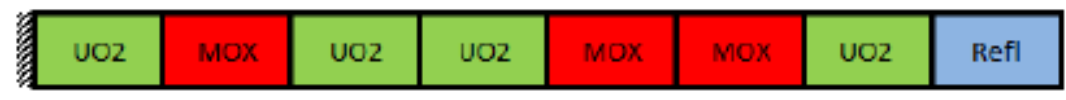

Figure 4.6: MOX 1 Core Layout

The multiscale regions used for the MOX1 core are identical to the LEU core, 4.4, because the assembly with the peak power is also at the center of the core.

Results for MOX 1 Core 
The addition of MOX assemblies into the core adds heterogeneity that is difficult to model with the current methodology. The assumption that the few group cross-sections and form functions can be generated from an infinite assembly creates issues at the LEU MOX interface because the assemblies are very different. Figure 4.7 shows the pin errors in the diffusion solution for the same energy case. Unlike the LEU core the average pin power errors are much larger and spikes in the pin error can be seen at all of the interfaces of LEU and MOX assemblies.

\section{Same Energy Results for MOX1 Core}

When the multiscale method is applied to the center assembly of the MOX 1 core, all methods reduce the pin power errors. Table 4.2.3 shows the figures of merit for the two multiscale methods.

\begin{tabular}{|r|c|c|c|c|c|c|}
\hline & $\tau$ & $\Delta \mathrm{k}$ & $E^{\text {peak }}$ & $E^{\text {max }}$ & $E^{\text {RMS }}$ & $E^{\text {RMS shape }}$ \\
\hline 2G Diffusion & 29.3 & 59.8 & $2.69 \%$ & $9.47 \%$ & $2.01 \%$ & $1.50 \%$ \\
\hline 2G Fixed Source & 9.8 & 59.8 & $0.54 \%$ & $8.10 \%$ & $1.63 \%$ & $0.33 \%$ \\
\hline 2G Albedo & 6.0 & -114.4 & $1.09 \%$ & $8.10 \%$ & $1.75 \%$ & $0.86 \%$ \\
\hline
\end{tabular}

The fixed source boundary method gives the best improvement on local quantities such as the peak pin error and the local pin power shape RMS. It also runs the fastest of the two methods. Figure 4.8 shows the spatial pin errors for diffusion, the fixed boundary source, and the albedo method.

All of the multiscale methods perform well for cases where the global and local solvers are both solved with the same energy group structure. The next step is to move toward the more realistic case where the local solver uses more energy groups than the global solution. 


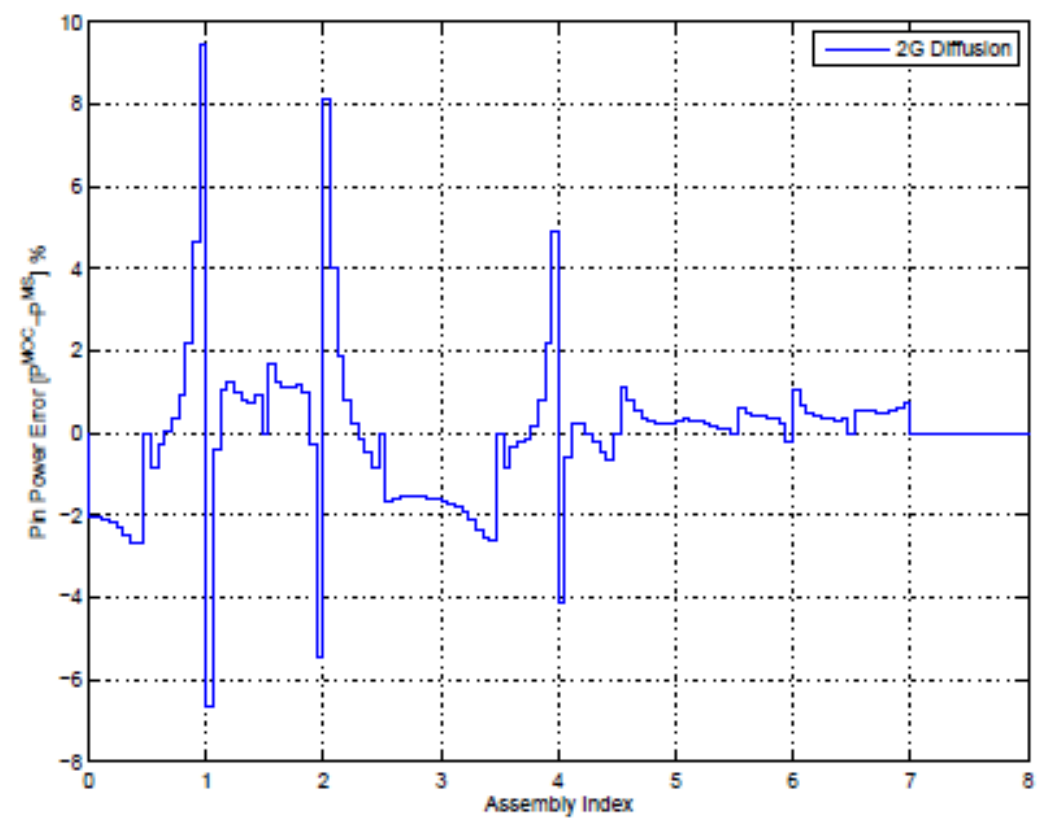

Figure 4.7: Pin Power Error in PPR for Same Energy MOX 1 Core

Mixed Energy Results for MOX1 Core

The mixed energy results without a buffer region are quite different from the results with the same energy structure. Table 4.2.3 shows the error in the PPR solution for the mixed energy multiscale methods without a buffer region.

The results with mixed energy groups are in considerable contrast to the results with the same energy groups. The fixed boundary source method is worse than PPR in most of the figures of merit. The albedo method maintains a better solution than diffusion. In order to understand why the fixed boundary source method is worse, figure 4.9 shows the spatial distribution of the pin power error for PPR, the fixed boundary source method, and the albedo method. From this figure the main source of error can be seen to occur at the interface. For the fixed source boundary condition, the source of the error is the assumption that the precalculated energy shape is sufficient to project the flux. As indicated this assumption results in large errors in the pin powers near the boundary. 


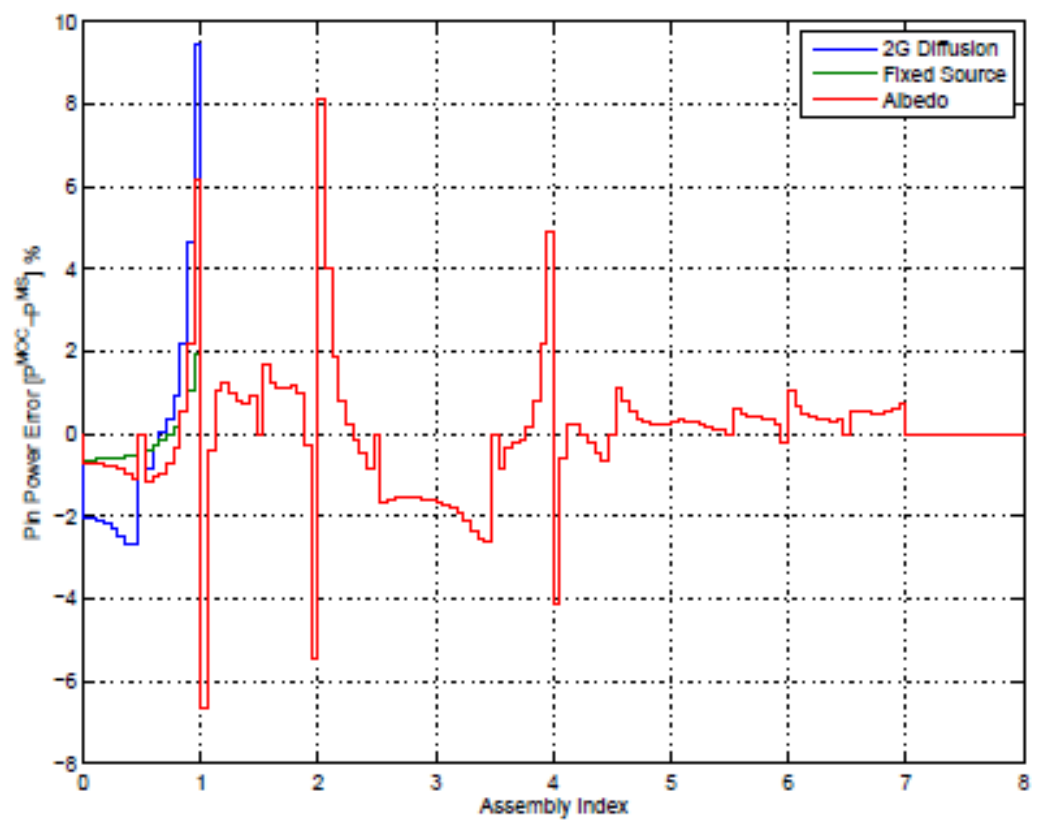

Figure 4.8: Pin Power Error in Multiscale for Same Energy MOX 1 Core

Table 4.3: FOM Improvements over PPR for Mixed Energy MOX 1 Core without Buffer Region

\begin{tabular}{|r|c|c|c|c|c|}
\hline & $\tau$ & $E^{\text {peak }}$ & $E^{\max }$ & $E^{\mathrm{RMS}}$ & $E^{\mathrm{RMS} \text { shape }}$ \\
\hline Diffusion & 256.6 & $-1.07 \%$ & $15.03 \%$ & $2.66 \%$ & $1.47 \%$ \\
\hline Fixed Source & 10.9 & $-6.24 \%$ & $40.96 \%$ & $4.88 \%$ & $5.84 \%$ \\
\hline Albedo & 7.6 & $-1.40 \%$ & $15.03 \%$ & $2.48 \%$ & $0.48 \%$ \\
\hline
\end{tabular}

Another interesting observation of the fixed boundary source solution is that the pin power error is large at the interface but a few pins away from the interface have errors comparable to the diffusion solution. This seems to suggest that a region where the solution was allowed to develop but was not applied to the final solution would considerably increase the accuracy of these methods. In this work, this region is called the buffer region. The next set of results show a buffer region is applied to both the albedo and fixed boundary source regions to provide a better angle and energy distribution of the flux. 


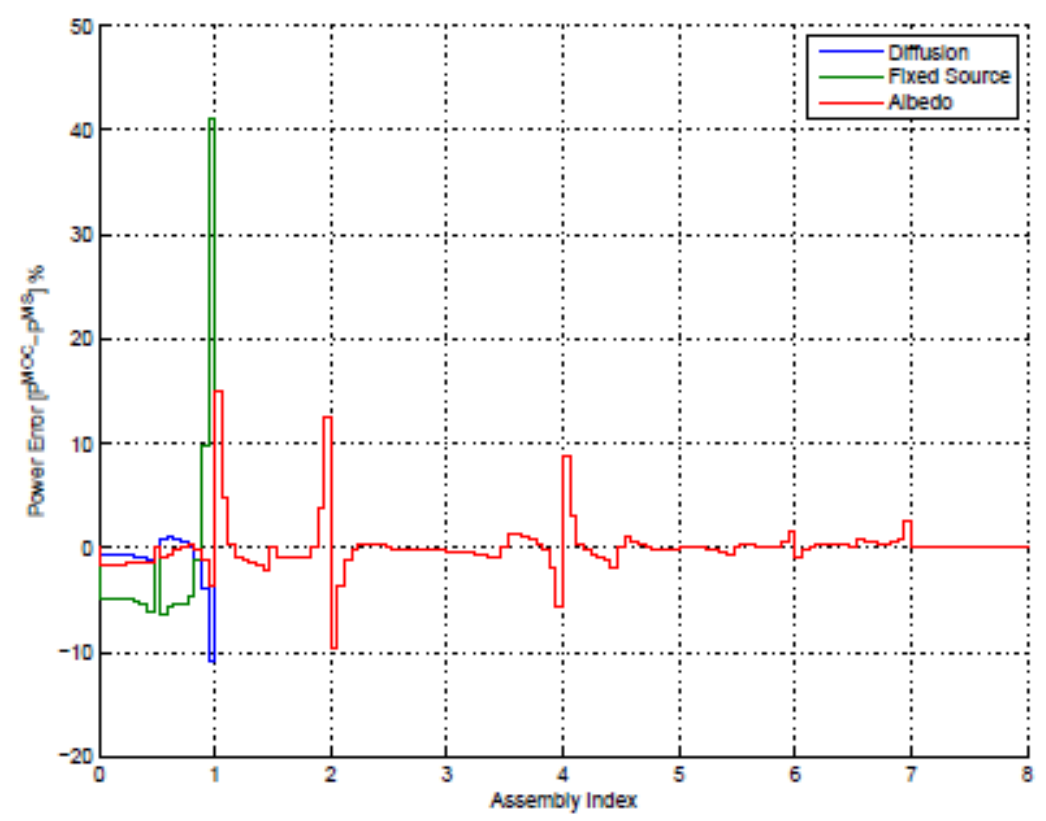

Figure 4.9: Pin Power Error in Multiscale for Mixed Energy MOX 1 Core without Buffer

The inclusion of a buffer region in the post-refinement methods increases the computational burden but provides a region where the boundary errors can adjust to the local region geometry and energy group structure without negatively impacting the solution. The method that seems to gain the most from the buffer region is the post-refinement fixed boundary source problem. It was shown that the boundary condition causes significant errors in the pins closest to the boundary. The buffer region eliminates these errors by ignoring the solution in that region. Figure 4.10 shows the error when a buffer region is included in the local computation. In all cases shown here, the buffer region is the size of a single assembly.

For both post-refinement methods the buffer region increases the accuracy of the multiscale calculation. Even though the albedo solution was much better without the buffer region, the two methods produce an almost identical result when the buffer region is added.

The next extension is to increase the region of interest to two assemblies. The extension of the region of interest will eliminate the dependency of the assembly error. Instead, the power generated in the entire region of interest is used to determine the local pin powers. In general the error associated with a larger region is less than the error of a smaller region. This is because the power is normalized so as the region grows larger, the average error approaches zero. There are cases where the local diffusion error of a single assembly is small but the surrounding assemblies have larger errors. In this case making the region of interest larger can increase the region average error and negatively impact the pin power errors. This effect is not seen in either of the 
MOX cores but was observed in the LEU core. Figure 4.11 shows the error in both methods over diffusion when another assembly is added to the region of interest.

In both cases, the error is greatly reduced by expanding the region of interest.

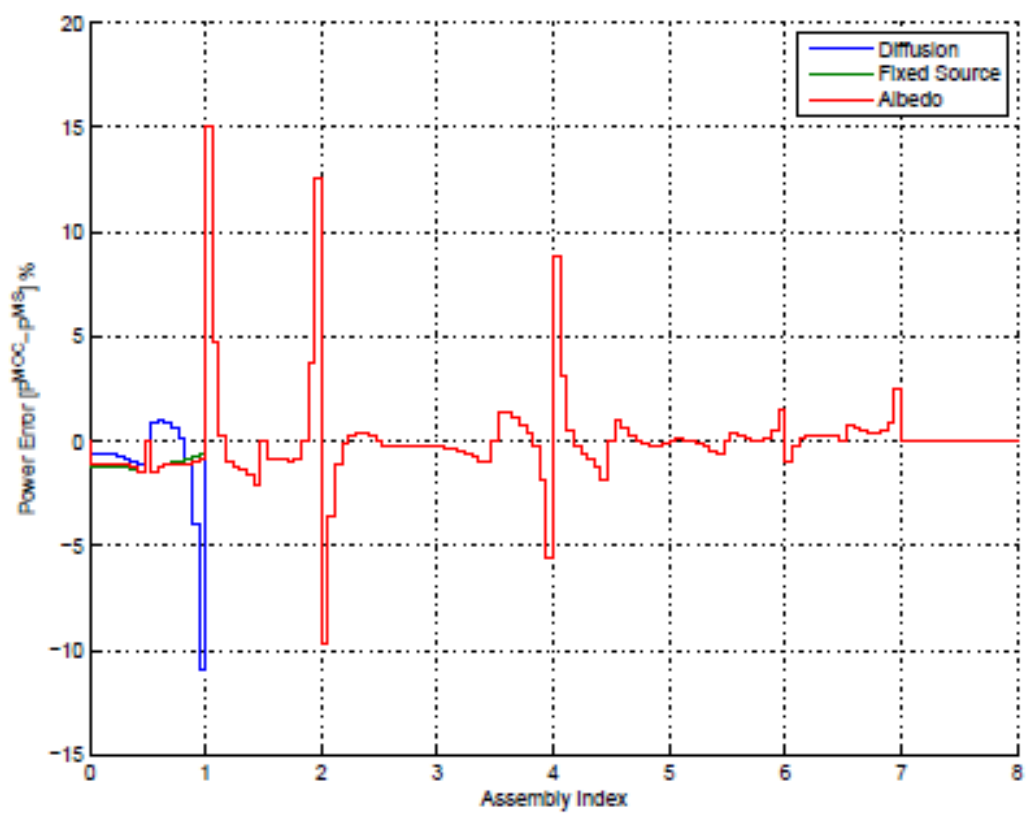

Figure 4.10: FOM Improvements over PPR for Mixed Energy MOX 1 Core with Buffer Region

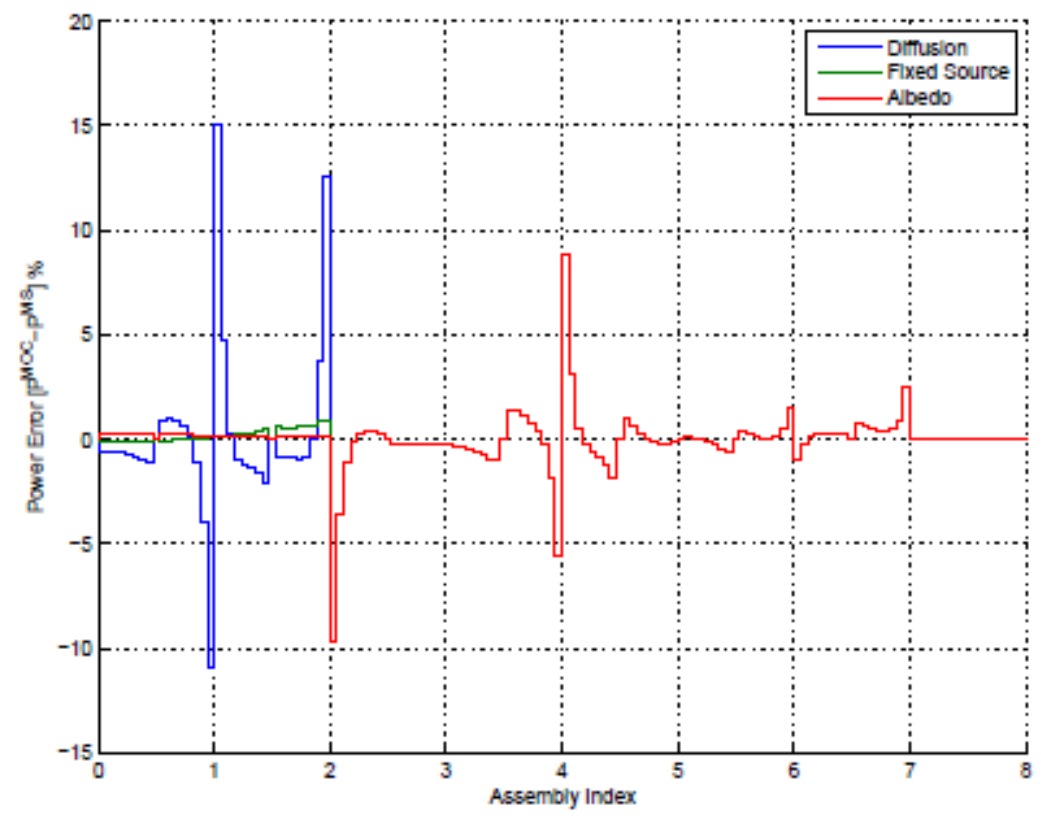

Figure 4.11: FOM Improvements over PPR for Mixed Energy MOX 1 Core with Increased Region of Interest and Buffer Region 
Another natural question is whether the buffer region is needed for the albedo method. For the single assembly without a buffer region, the albedo method results show a good improvement in the solution. It is advantageous if a buffer region is not needed because the computational requirements are reduced. The post-refinement methods for the albedo case are repeated only if the buffer region is added to the region of interest. The two assembly region of interest problem can be performed with computational requirements similar to the single assembly problem with a buffer region and the three assembly region of interest problem can be performed with computational requirements similar to the two assembly problem with a buffer region. The results of these cases are shown in Figure 4.12.

It can be quickly seen that the boundary condition for the two assembly region of interest causes slightly larger pin power errors than the diffusion solution at the boundary. These errors are considerably less than pin errors introduced by the fixed source problem. The remainder of the solution shows considerable improvement over the single assembly. The three assembly region of interest has a worse solution than the equivalent computational demand case with a two assembly region of interest and a buffer region. This is caused by a cancellation of assembly power errors in the first two assemblies. The addition of a third assembly increases the region of interest average power error and makes the powers worse. It should also be noted that the error in the pin power shape of both cases are identical for the assembly containing the peak pin so increasing the region of interest is only an effort to try to reduce the effect of individual assembly errors and does not improve the shape. Ultimately it seems to be better to include a buffer region instead of increasing the region of interest to eliminate the potential impact of the boundary condition onto the global pin power solution.

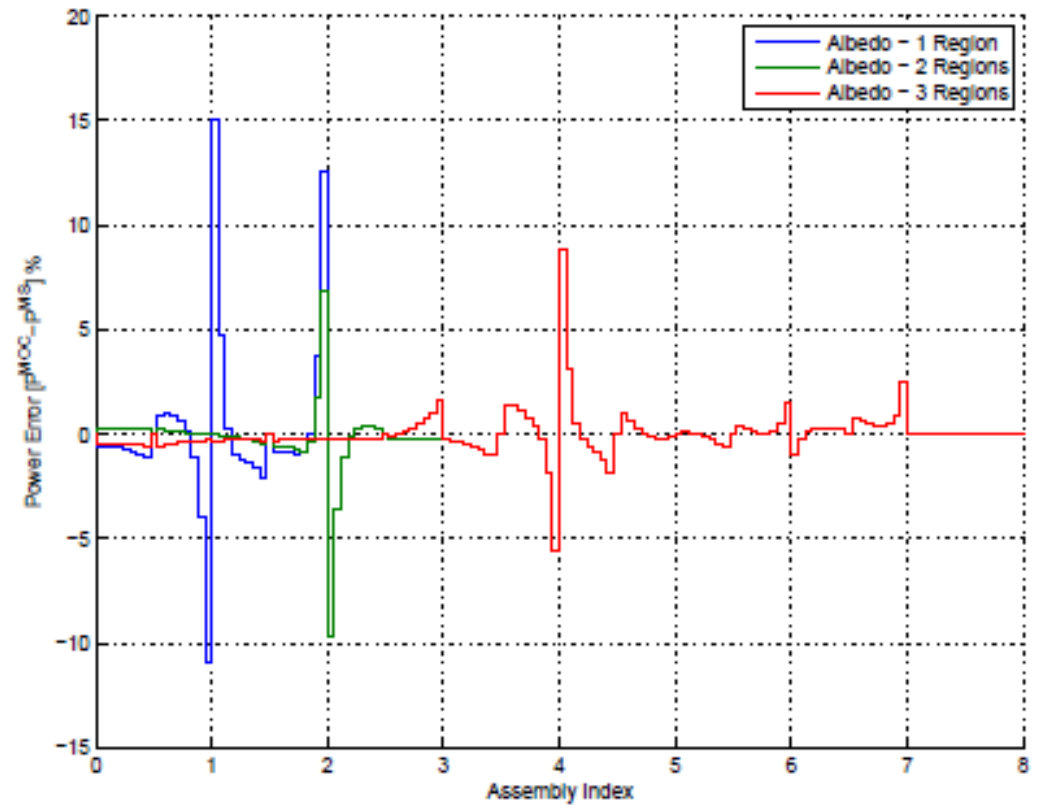

Figure 4.12: Pin Power Error in Multiscale Mixed Energy MOX 1 Core with No 


\section{Buffer Region}

The method is also analyzed with the 33 group cross-section library. The size of this library is more consistent with the size of standard cross-section libraries used for transport calculations. It is important to determine if the post-refinement method can handle projections from 2 to 33 energy groups. Cases are run with and without a buffer region using both the fixed boundary source and albedo methods. In both cases, the buffer region is necessary to accurately capture the difference in the spectral effects.

\section{MOX2 Core}

The second MOX core is designed to test the effect of the multiscale methods in the case where the peak assembly is not on the left boundary. This will require a two sided multiscale interface around the assembly with the peak power instead of a single sided interface. Again, four LEU and three MOX assemblies are laid out in the core with a single reector assembly is located on the right side. Figure 4.13 shows the layout of the assemblies.

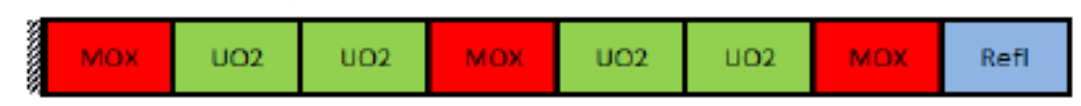

Figure 4.13: MOX 2 Core Layout

\section{Multiscale Regions}

The MOX 2 core has a slightly different layout for the multiscale regions because the peak power is not located near the boundary. Figure 4.14 shows the region of interest and buffer regions that the post-refinement cases will use.

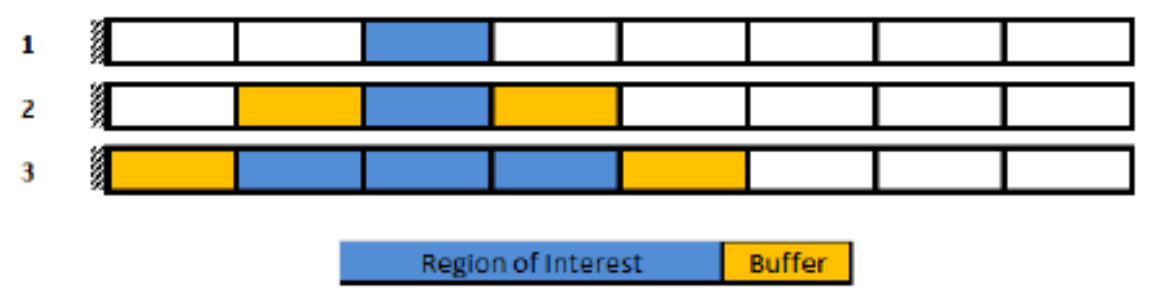

Figure 4.14: Multiscale Regions for 1-D MOX 2 Core

The first multiscale region is simply a region of interest in the assembly with the peak pin power. The second multiscale region adds a buffer region on both sides of the peak assembly. The last multiscale region spans five assemblies with a buffer region in the outer assemblies.

Results for MOX2 Core 
The second MOX core is introduced to ensure that the multiscale methods work if the region of interest does not coincide with a boundary. In this case, the boundary condition must be estimated for both the left and right face of the local domain.

When a buffer region is added, it is added to both sides, and when the region of interest is expanded, it is expanded in both directions. The MOX 2 core has a very similar behavior as the MOX 1 core. For the same energy cases, all methods show improvement over the diffusion solution. In the more practical mixed energy case, the fixed boundary source method without a buffer region does not perform well. The albedo method performs well for all cases. The fixed boundary source method with a buffer region also performs well. Since two boundary conditions must be approximated instead of one, it makes sense that the improvements are less than the MOX 1 core. The figures of merit are summarized in table 4.4.

Table 4.4: Figures of Merit for MOX 2 Core

\begin{tabular}{|r|c|c|c|c|c|}
\hline & $\tau$ & $E^{\text {peak }}$ & $E^{\text {max }}$ & $E^{\text {RMs }}$ & $E^{\text {RMS shape }}$ \\
\hline Diffusion & 256.6 & $-1.07 \%$ & $15.03 \%$ & $2.66 \%$ & $1.47 \%$ \\
\hline Fixed Source - MS 1 & 10.9 & $-6.24 \%$ & $40.96 \%$ & $4.88 \%$ & $5.84 \%$ \\
\hline Fixed Source - MS 2 & 2.8 & $-1.54 \%$ & $15.03 \%$ & $2.46 \%$ & $0.04 \%$ \\
\hline Fixed Source - MS 3 & 0.8 & $-0.14 \%$ & $9.63 \%$ & $1.49 \%$ & $0.04 \%$ \\
\hline Albedo - MS 1 & 7.6 & $-1.40 \%$ & $15.03 \%$ & $2.48 \%$ & $0.48 \%$ \\
\hline Albedo - MS 2 & 3.1 & $-1.46 \%$ & $15.03 \%$ & $2.46 \%$ & $0.04 \%$ \\
\hline Albedo - MS 3 & 2.6 & $0.28 \%$ & $9.63 \%$ & $1.49 \%$ & $0.00 \%$ \\
\hline
\end{tabular}

Overall the one dimensional results provide several interesting insights about the behavior of the multiscale methods. Using these insights, the number of two dimensional tests is reduced to only consider the post-refinement albedo method with mixed energy.

Two Dimensional Analysis

The two reactors analyzed here are both based on a 156 assembly core modeled with quarter core symmetry. The first core uses a uniformly enriched LEU assembly surrounded by a water reflector. The second core contains both LEU and MOX assemblies loaded in an arrangement to atten the power shape and push the peak pin power away from the center of the core. There are $64 \mathrm{MOX}$ assemblies and $92 \mathrm{LEU}$ assemblies loaded into the reactor.

Assembly Layouts

The layout of the two dimensional reactors is based on the assembly design from the C5G7 benchmark. The assembly is designed with an array of $17 \times 17$ pins; 264 are fuel pins, 24 are guide tubes for control rods, and the center pin contains a fission chamber. The geometry is specified by the benchmark but key dimensions are repeated in table 4.5 .

Table 4.5: Two Dimensional Assembly Geometry 
Assembly Pitch $21.42 \mathrm{~cm}$

Pin Pitch $1.26 \mathrm{~cm}$

Fuel Radius $0.54 \mathrm{~cm}$

The LEU assembly is a uniform arrangement of fuel pins with the same enrichment. The assembly layout is shown in figure 4.15. The MOX assembly has three enrichment zones and the layout is shown in figure 4.16. Lastly a reflector assembly is defined to have an assembly pitch and filled with the moderator material. In the case where control rods are inserted into the assembly, the guide tube is replaced by the control rod material with the same radius as the fuel pin.

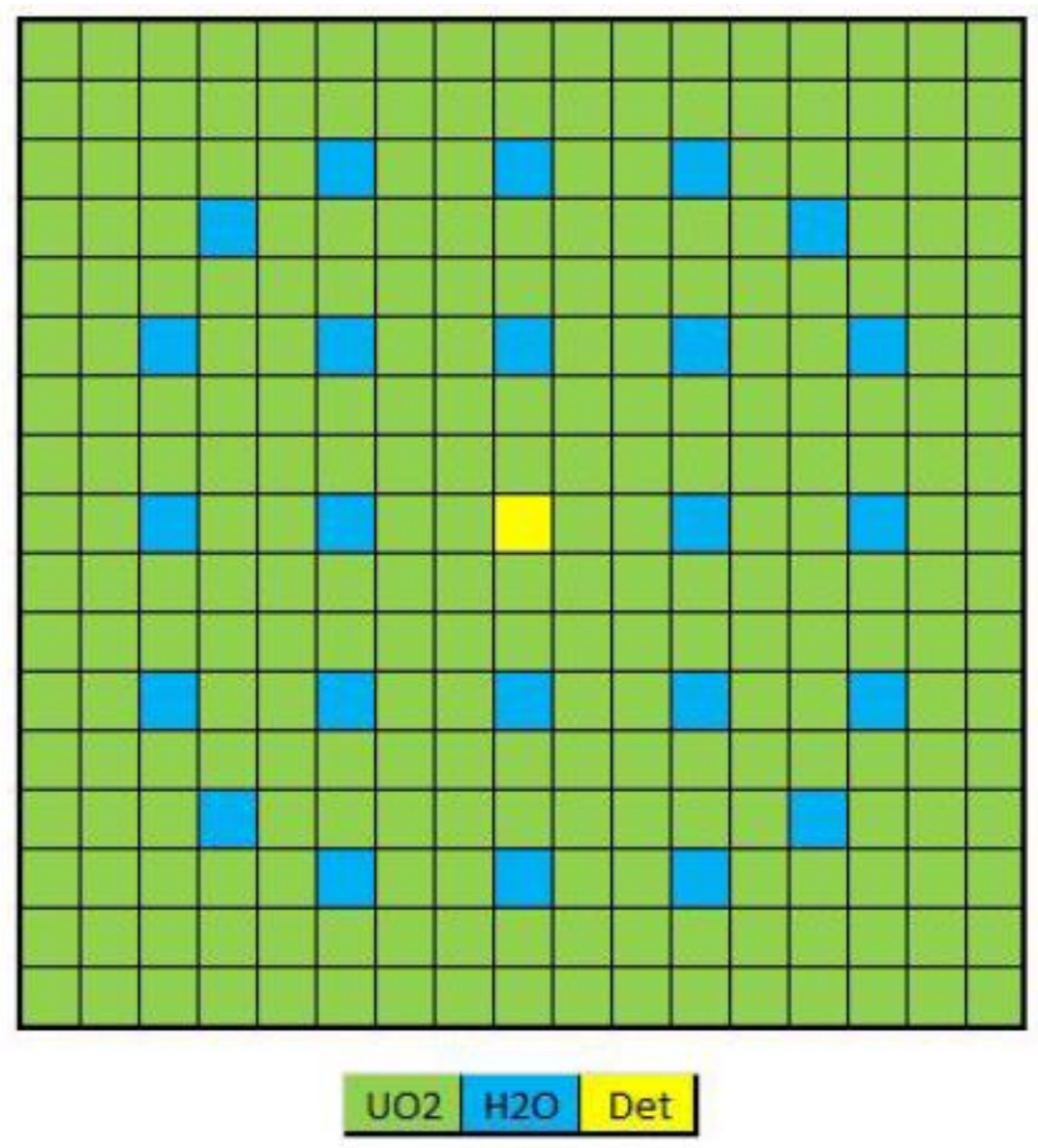

Figure 4.15: 2-D LEU Assembly 


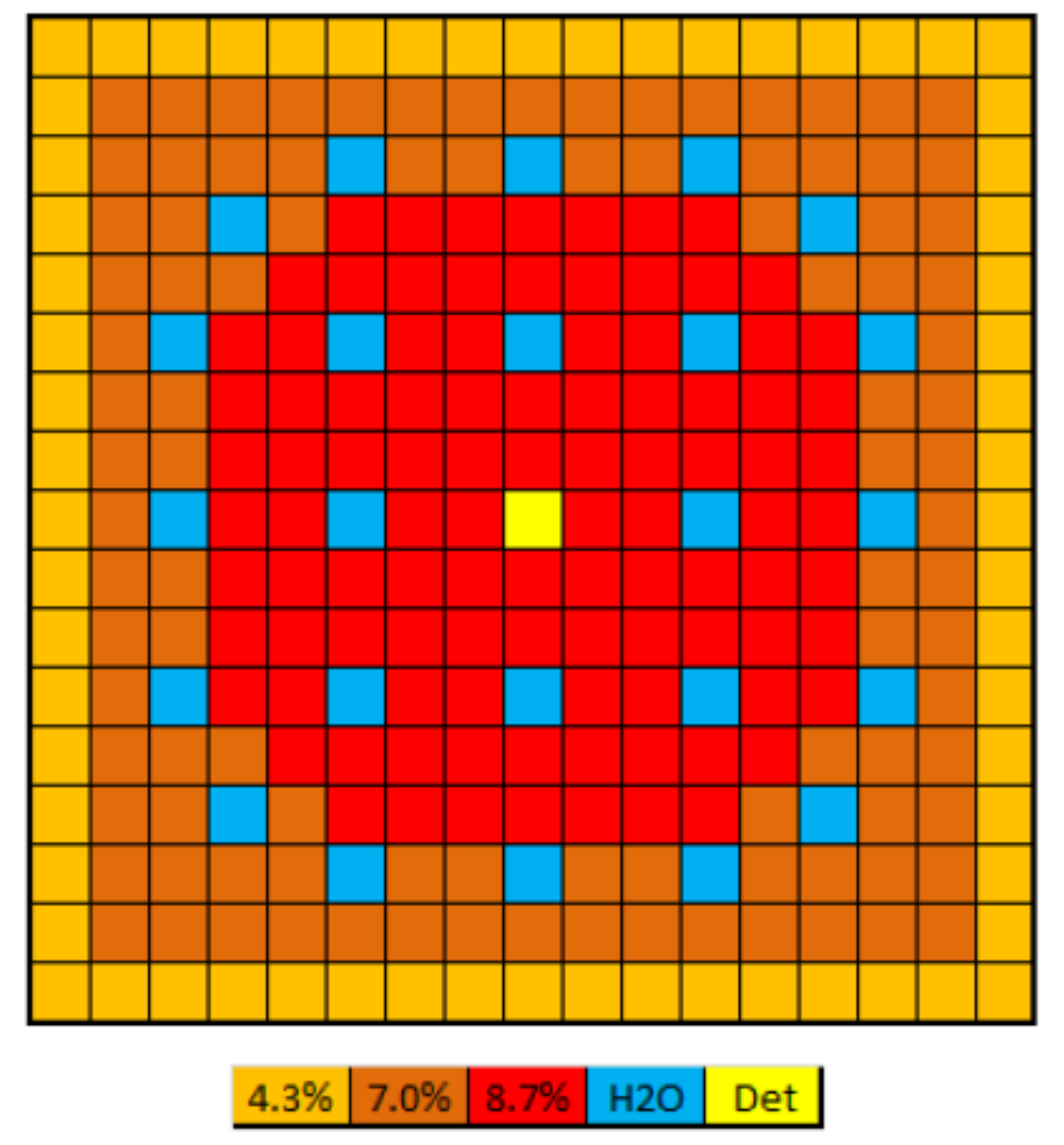

Figure 4.16: 2-D MOX Assembly

LEU Core

The LEU core is loaded completely with the same fuel type in all locations. The computational model only considers one quarter symmetry which places reflective boundary conditions on the left and bottom boundary of the computational domain. The right and top boundaries have a zero incoming current boundary condition. The LEU core is shown in Figure 4.17. 


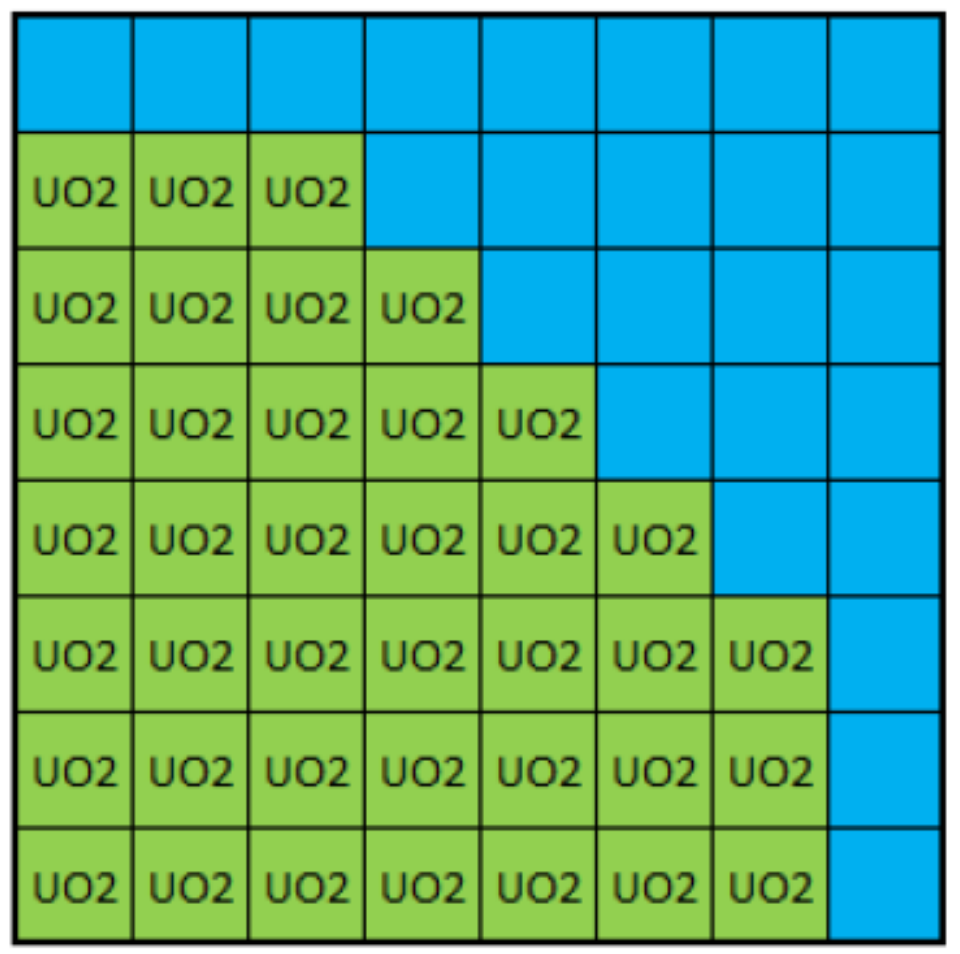

Figure 4.17: 2-D LEU Core Layout

\section{Multiscale Regions}

Three multiscale regions are defined for each core. Since the peak power is in the center assembly for the LEU core, the multiscale regions are based around the symmetry boundary. Figure 4.18 shows the three trust regions. 


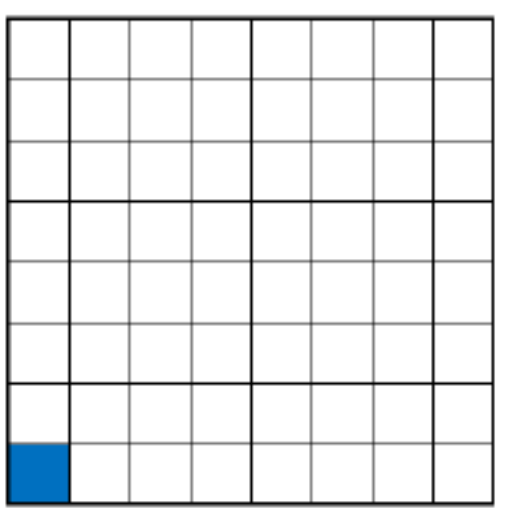

1

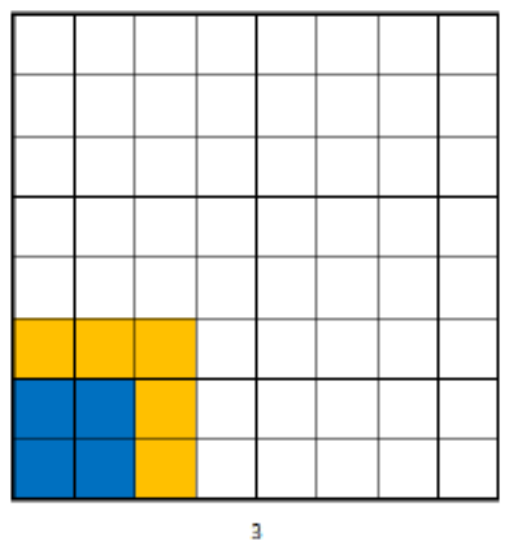

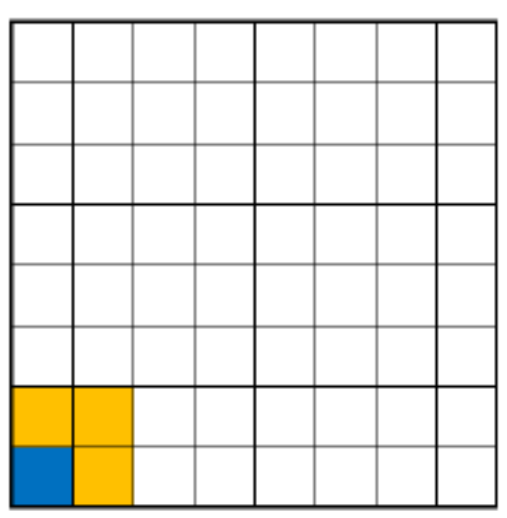

2

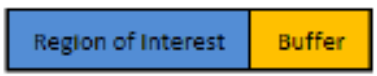

Figure 4.18: 2-D LEU Core Multiscale Regions

The first multiscale region is a single assembly with no buffer region surrounding it. The second multiscale region is a single region of interest with buffer region surrounding it. The last is a two by two assembly region of interest with a buffer region surrounding it.

\section{Results for LEU Core}

The two dimensional LEU core is a designed to test the initial performance of the postrefinement albedo method in two dimensions. As with the pure LEU case in one dimension, the diffusion solution with PPR already produces very good results. The major source of error occurs at the core reflector interface where pin errors of approximately 10 percent are found. Figure 4.19 shows the spatial distribution of pin power error in the diffusion solution. 


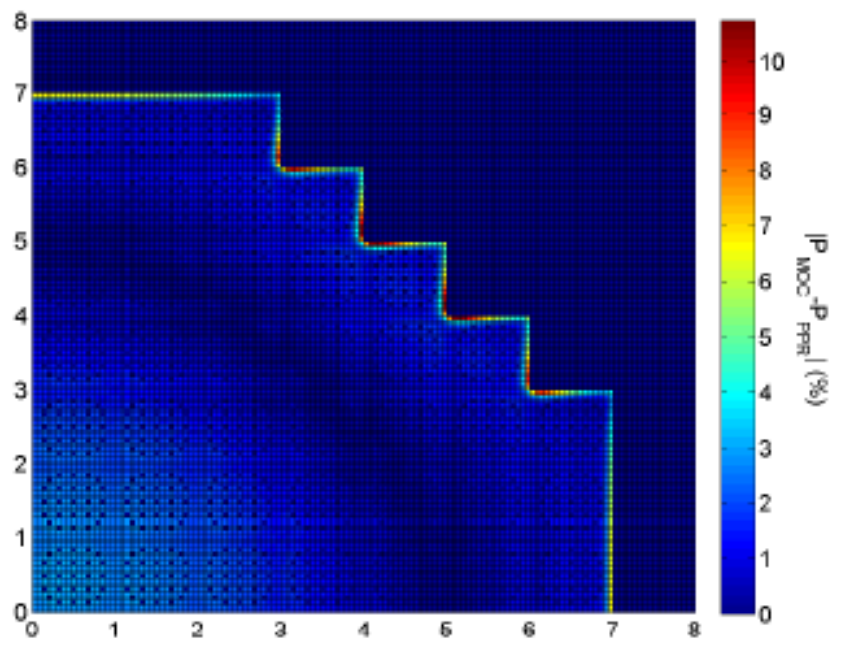

Figure 4.19: Pin Power Error in PPR for 2D LEU Core

The post-refinement albedo method shows marginal improvements in the solution for all of the trust regions. However, the error in the pin power shape increases slightly over the diffusion solution. This means that the error in the peak pin power is completely driven by the error in the assembly powers which the post-refinement methods cannot change. The error in the assembly powers are shown in Figure 4.20. The region of interest would have to be expanded several assemblies in order for the region average error to become small.

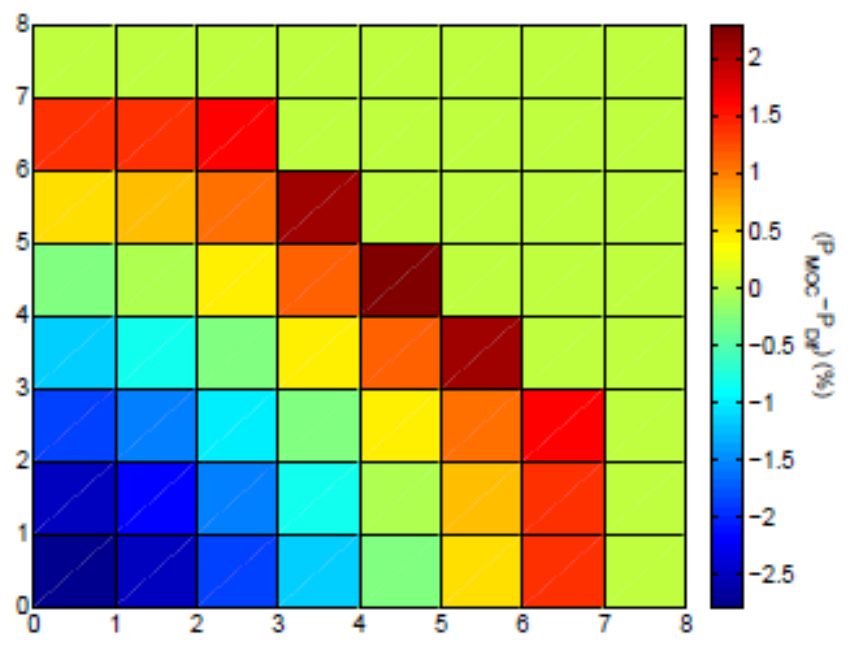

Figure 4.20: Assembly Average Power Error in Diffusion Solution for 2D LEU Core

\section{MOX Core}

The MOX core, Figure 4.21, is very similar to the LEU core except 16 of the LEU assemblies are replaced by MOX assemblies. MOX assemblies are loaded in the center of the core to push 
the peak power away from the center. This is done in order to test the multiscale method for a peak power away from the symmetry boundary.

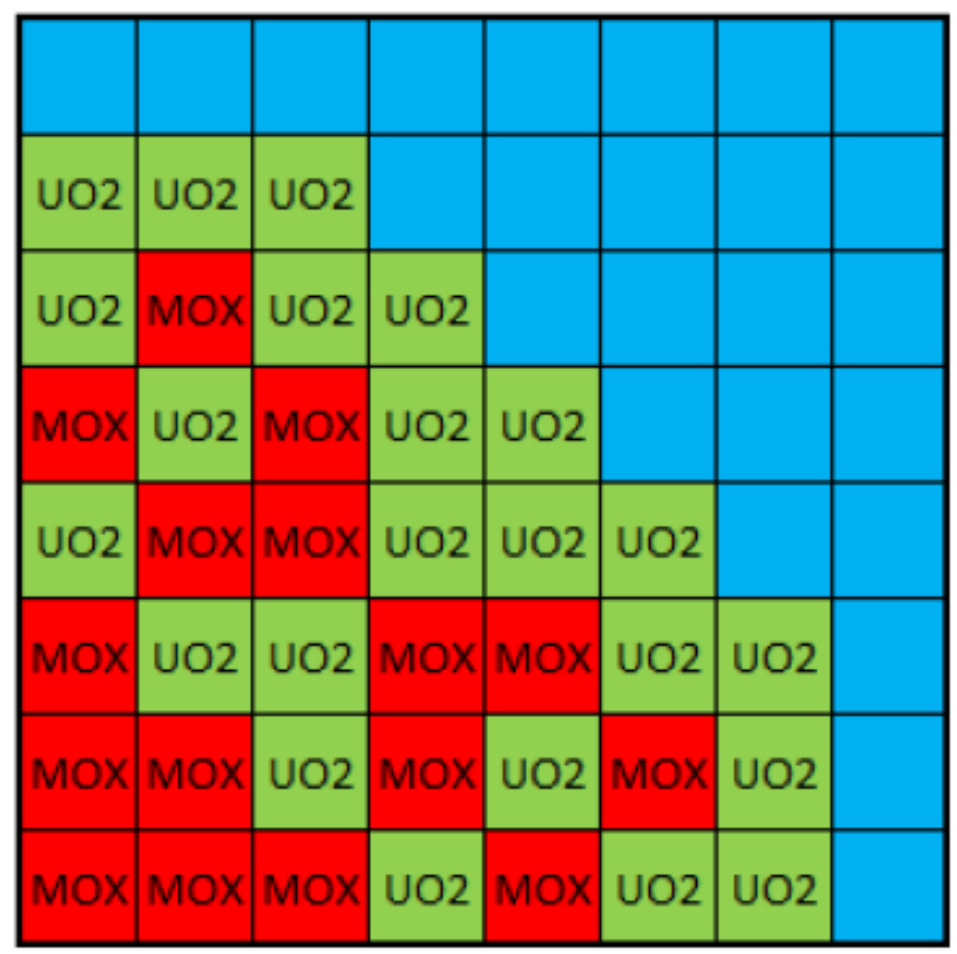

Figure 4.21: 2-D MOX Core Layout

Multiscale Regions

The MOX core has the peak power in the $(3,3)$ assembly and the region of interest surrounds this point as shown in Figure 4.22. The first multiscale region is only the single assembly without a buffer region. The second multiscale region surrounds the assembly with a buffer region. The last multiscale region expands the region of interest to include 9 assemblies and surrounds that region with a buffer region. 


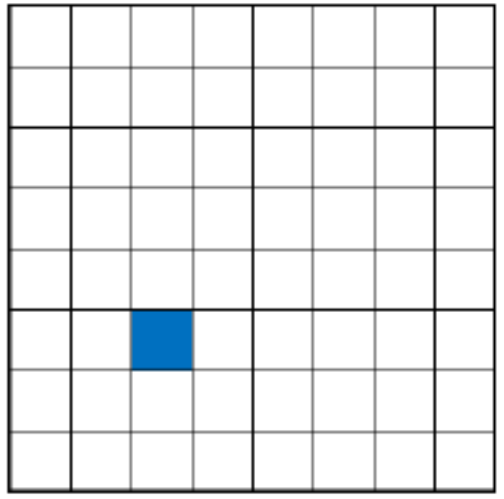

1

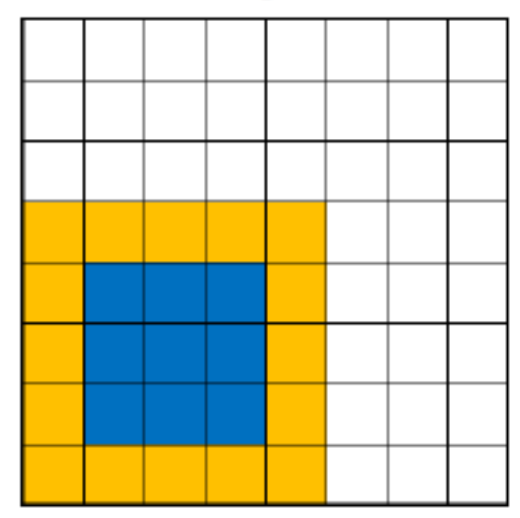

3

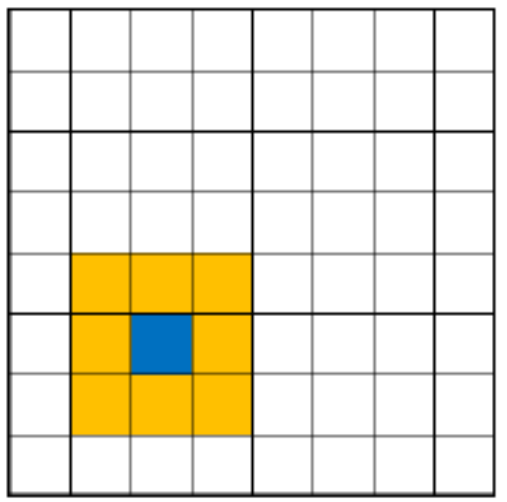

2

\section{Region of Interest Buffer}

Figure 4.22: 2-D MOX Core Multiscale Regions

Results for MOX Core

The two dimensional MOX core adds a considerable amount of heterogeneity that diffusion with PPR does not handle well. The error in the pin powers are shown in Figure 4.23. Errors occur at all of the LEU MOX interfaces with pin errors ranging from about 10 to 15 percent. At the core reflector interface, pin errors can be found ranging up to 25 percent. Even with large errors at the assembly interface, the pin power errors in the center of the assembly are small. 


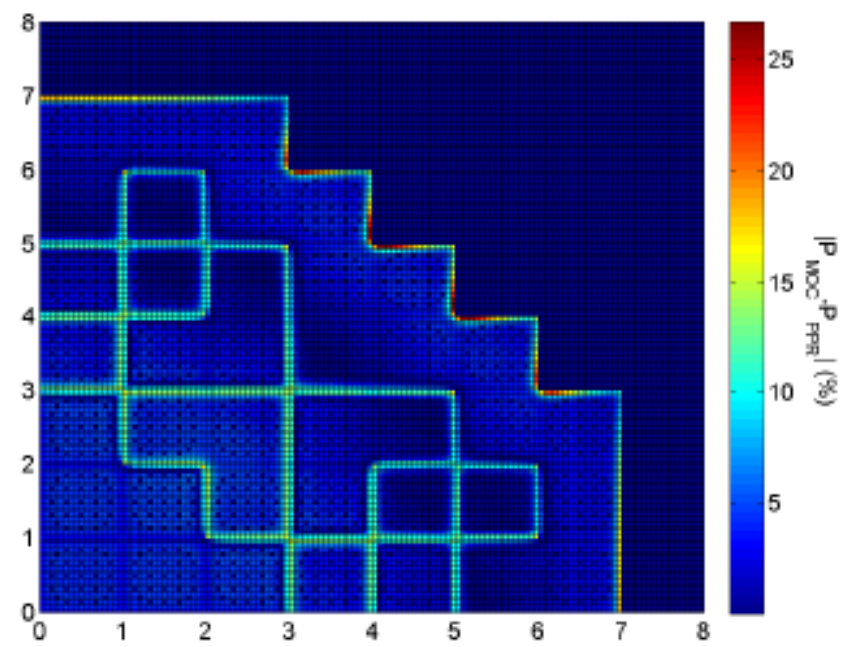

Figure 4.23: Pin Power Error in PPR for for 2D MOX Core

The multiscale method applied to the single assembly without a buffer region slightly improves the pin power errors. Adding a buffer region around the single assembly improves the shape of the solution but the error in the assembly power error makes the peak pin power error worse than PPR. When the multiscale is applied when the region of interest is increased to a three by three set of assemblies surrounded by a buffer region, the pin power errors are greatly reduced in the entire region of interest as shown in Figure 4.24.

Just as in the one dimensional cases, increasing the region of interest decreases the error in the region wise power. Figure 4.25 shows the assembly power errors in the diffusion solution for the MOX core. The assembly with the peak power is about 5 percent below the MOC assembly average power but the surrounding assemblies are a mix of errors above and below the MOC solution. The average error for the three by three region of interest is $2: 5$ percent.

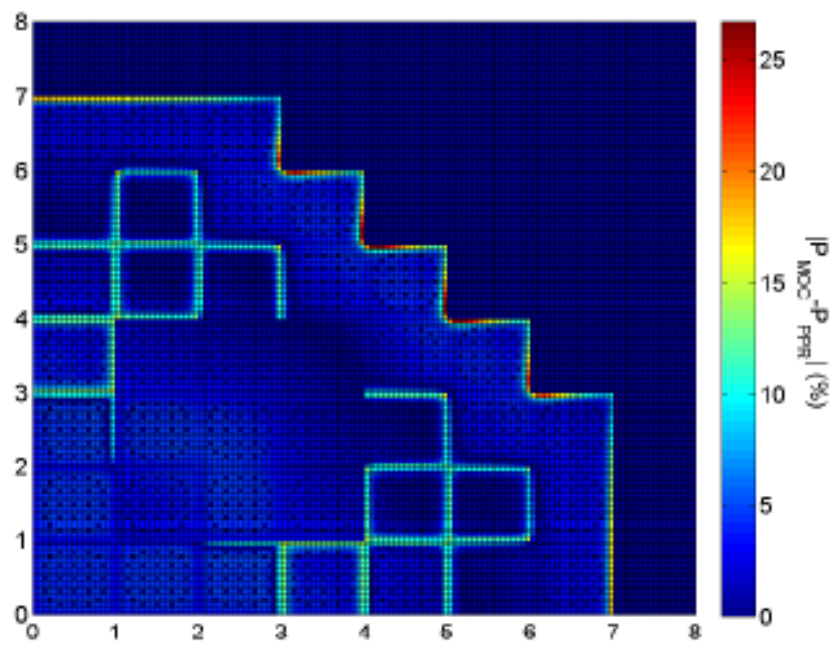

Figure 4.24: Pin Power Error of Multiscale Solution for 2D MOX Core with Increased 
Another case that was considered is the three by three region of interest without a buffer region. As seen in the one dimensional results, it is possible to obtain very good results using the albedo method without a buffer region but there are some cases where not having a buffer region can cause local pin errors. Since the pin errors on the periphery are already relatively large, it may be worthwhile to remove the buffer region to reduce some of the computational burden. Figure 4.26 shows the resulting pin power error for the case where the buffer is removed from the expanded region of interest. The results from this case are improved over the case with a single assembly with a buffer region which has similar computational cost but the expanded region of interest with a buffer layer still has better results in all categories. Although these results show that the case without a buffer region can provide very good results, it is important to note that when a buffer region is not used, the results appear to vary case by case. If the assembly power error distribution is known, it may be practical to decide if a buffer region is needed. In the end, the user should be very cautious when not using a buffer region because the influence of the boundary condition to pins near the boundary could give solutions worse than the original diffusion solution with pin power reconstruction (PPR).

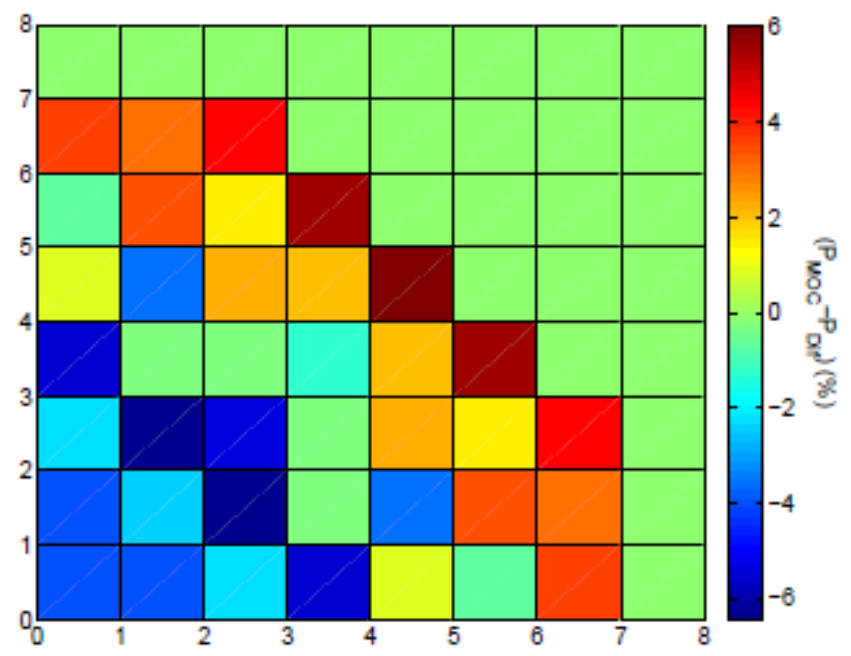

Figure 4.25: Assembly Average Power Error in Diffusion Solution for 2D MOX Core 


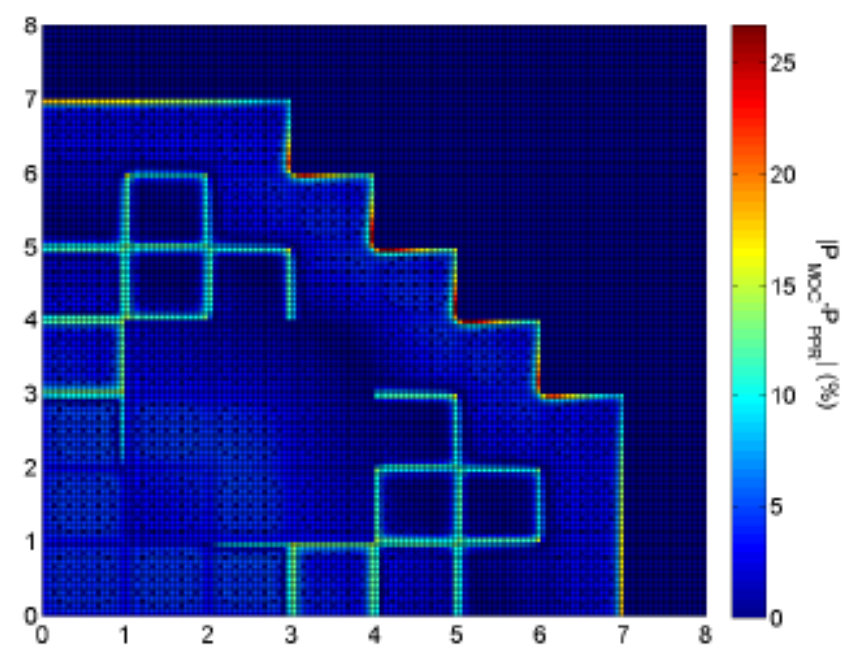

Figure 4.26: Pin Power Error in Multiscale Solution for 2D MOX Core with Increased Region of Interest and No Buffer Region

\section{Discussion of Post-Refinement Multiscale Methods}

The results given in the previous section and in the appendix A provide important insights into the behavior of the post-refinement multiscale method. Several conclusions can be drawn from the information obtained and the results will be analyzed to offer a set of best practice guidelines when developing post-refinement multiscale methods for reactor analysis.

\section{Same Energy and Mixed Energy Results}

The issue of energy projection is a crucial question of this work. In general, diffusion codes are designed to solve 2 , 4 , or 8 energy group calculations but the lattice codes that are used to generate cross-sections in the multiscale approach are solving many more energy groups ( 50200). The projection in energy is a necessary step for the multiscale method to work correctly. In the one dimensional results, the same energy cases were performed to test the impact of angular coupling. For the same energy cases, all of the cores and multiscale configurations analyzed produced results that were improved over the diffusion solution with pin power reconstruction. This means that the angular dependence on the solution is secondary to the spatial and energy dependence. Since the same energy grid was used, no projection was necessary in the energy variable.

The mixed energy results were then considered and the results were very different. The postrefinement fixed boundary source method provided results that were considerably worse than the diffusion solution with PPR, especially near the local domain boundary. The albedo results showed modest improvements over diffusion by assuming the albedo is a constant function of 
energy for the projection. Without the use of buffer regions, the energy projection methods discussed here can not sufficiently describe the energy distribution of neutrons at the interface.

\section{Impact of Buffer Region}

The use of a buffer region provides a region where the flux can adjust to all of the differences in the local solution; different energy grid, different angular grid, and discrete pins instead of homogenized cross-sections. Most importantly the buffer region appears to be able to filter out the impact of boundary conditions that have errors.

From the results it appears as though a few pin pitches is a sufficient buffer to obtain a correct energy distribution but because of practical implementation issues in the test code used for these problems only assembly size buffer regions could be used. The thickness of a buffer for these methods is not limited by the method, but by the code in which the method is implemented.

The one-way coupling methods showed consistent improvement when the buffer was added but if computational costs are too high, the albedo method can be used without a buffer region for most problems. Although the albedo method produces reasonable results for the case without a buffer region, the resulting pin powers can be worse than the PPR pin powers. For this reason it is always suggested that a buffer region be used for one-way coupling methods.

\section{Comparison of Post-Refinement (One Way Coupling) Methods}

The post-refinement methods both suffer from the same flaws mentioned in the previous section. In order to choose the best method the fixed boundary source and albedo methods need to be looked at in greater detail. The first category to consider is the ease of implementation into existing high order methods. Many of the target high order codes (DeCART, DENOVO, and MCNP) considered for implementation already have the functionality to solve an eigenvalue problem given an albedo boundary condition. In many cases, the albedo boundary condition is not as generalized as this method requires. Development issues would involve making the albedo dependent on space and energy at the pin cell level. The work required to do this is modest. Some of the target lattice codes also have the ability to define an incoming angular flux. Again, modification would need to be made to define the angular flux as a function of space, angle, and energy on the entire domain. Another minor modification would be for the fixed source solver to accept an arbitrary eigenvalue that would be determined by the global solver. Ultimately, the implementation of the albedo method appears to be a better fit for the lattice codes considered for implementation.

A second consideration is execution time. The computational cost for both methods is approximately equal for the same size domain. The main difference is that for the albedo 
method, it is not essential to have a buffer region. The removal of the buffer region makes the method much more computationally advantageous.

A third consideration is the sensitivity of the method to errors in the input data. The global solution has inherent errors in the solution and thus the boundary conditions of the local solver derived from the global solution also have errors. It is useful to understand how these errors affect the solution. To better understand the difference in the way the fixed boundary source and albedo methods propagate errors, a sensitivity analysis was performed to determine which method is more stable.

\section{Assessment of the Embedded Multiscale Method}

Unlike the post-refinement method, the embedded method allows the global solution to adjust to the refined local solution. Since the global solution is altered, it can be beneficial to define refined local regions where the standard nodal diffusion methods have problems; not just around the peak assembly. In this chapter the embedded multiscale method will be applied to several regions in the reactor. The first location that is considered is around the peak power assembly which are identical to the cases looked at with the post-refinement method. Another location that can have a significant impact on the global solution is the fuel reflector interface since calculating the correct leakage in the core can have a significant impact on the global tilt of the error. By embedding the solver into the reflector region, the leakage out of the system can be calculated more accurately. The last location that is considered is around a control rod which can be important since a strong absorber causes a significant dip in the local flux. Although the standard method can do reasonably well in predicting the worth of the control rod, the pin powers around the control rod can have significant errors.

One Dimensional Analysis

The same one dimensional cores used in the previous chapter are used to assess the performance of the embedded method. As noted in the descriptions of the assemblies, when a control rod is inserted into an assembly the center water hole is replaced with control rod material. Another difference between the post-refinement method and the embedded method is that multiple levels can be embedded into the calculation. This analysis will investigate the use of up to four levels of embedded solvers. The first level's solver is always the assembly homogenized two group diffusion solver. Three additional solvers are added to increase the accuracy of the embedded calculation; a fine group MOC solver, a fine group pin homogenized diffusion solution, and an intermediate group pin homogenized diffusion solver. The MOC solver will be placed where the most accuracy is desired with the other two solvers surrounding to decrease the effects of prolongation at the boundary. As in the previous chapter, a series of three 
multiscale calculations are performed. The first is with the MOC solver in the region of interest. The second adds the fine group pin homogenized diffusion solution on both sides of the region, and the last adds the intermediate group pin homogenized diffusion solver. In order to determine the choice of intermediate group structures to use between the two group diffusion solver and the fine group MOC solver, the impact of intermediate level energy group levels on the accuracy of the solution were evaluated using the MOX1 core. Figure ?? shows the spatial distribution of the pin power errors in the MOX 1 core for diffusion solvers with 2, 4, and 7 energy groups. The error in the two group assembly homogenized diffusion solver with pin power reconstruction ( $2 \mathrm{G}$ Diffusion in the Figure) is compared to the error in the pin homogenized diffusion solver with 2, 4, and 7 energy groups.

At the interface between dissimilar fuel assemblies the error is significantly reduced as the number of groups is increased. The difference in the assembly and pin homogenized two group cross-sections is very small and the impact of pin homogeneous cross-sections appears to be negligible in the one dimensional problem. The four group cross-section set captures the majority of the spectral effects but increasing to seven groups does show some additional improvement in the solution. Even with seven group cross-sections, the diffusion solution still has signiffcant errors at the MOX/LEU interfaces. These differences can be attributed to the effect of spatially homogenizing cross-sections using a single infinite assembly spectrum, as well as transport effects at the boundary. Because the seven group pin homogenized diffusion solver provides a considerably improved solution, it is used in the region surrounding the MOC region. In cases where the multiscale region needs to be expanded, the four group pin homogenized diffusion solver will be added.

\section{Embedded Region Around Peak Power Assembly}

Like the post-refinement method, the embedded method can be used to resolve local pin power peaking to obtain a more accurate prediction of the peak pin power. The multiscale regions used for the LEU and MOX1 cores is shown in Figure 5.2.

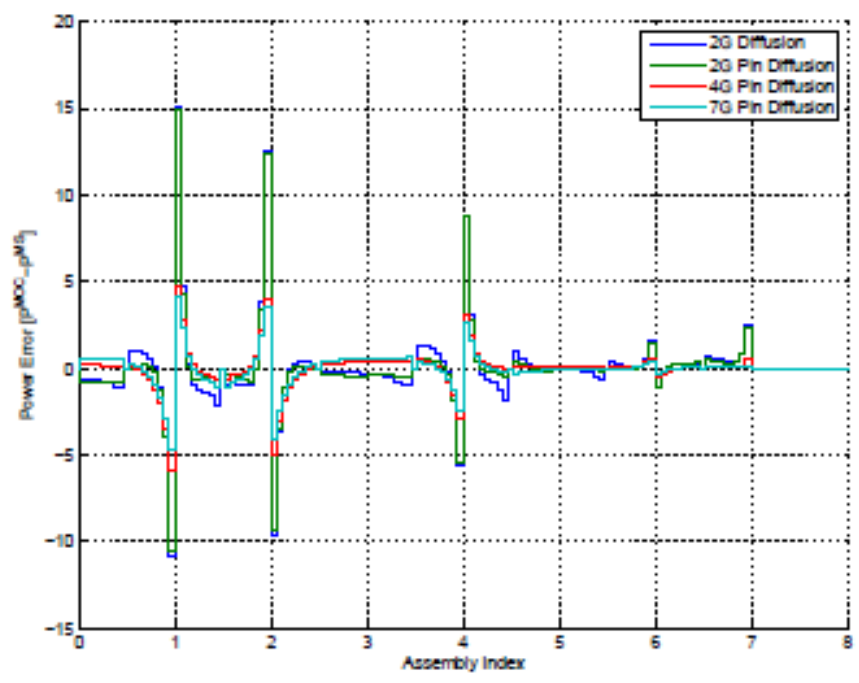


Figure 5.1: Pin Power Error Distribution for Diffusion Solvers

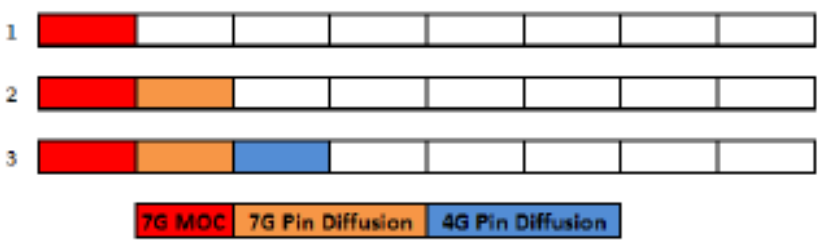

Figure 5.2: Embedded Multiscale Regions for LEU and MOX 1 Core

Table 5.1: Figures of Merit for LEU Core with Embedded Multiscale Method

\begin{tabular}{|c|c|c|c|c|c|c|}
\hline & $\bar{\tau}$ & $\Delta k$ & Bpant & $E^{\max }$ & $E^{1 \mathrm{MMS}}$ & 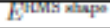 \\
\hline 2G Diffurion & 262.2 & 0.8 & $-1.00 \%$ & $5.13 \%$ & $0.79 \%$ & $0.03 \%$ \\
\hline MS - 1 & 5.6 & 1.3 & $-1.22 \%$ & $5.13 \%$ & $0.81 \%$ & $0.07 \%$ \\
\hline MS - 2 & 6.8 & 19 & $-1.33 \%$ & $5.14 \%$ & $0.85 \%$ & $0.01 \%$ \\
\hline MS - 3 & 6.4 & 2.4 & $-1.25 \%$ & $5.16 \%$ & $0.87 \%$ & $0.01 \%$ \\
\hline
\end{tabular}

\section{LEU Core}

The solution of the nodal diffusion solver predicts the pin powers of the LEU core very well. The multiscale results are shown in table 5.1. In all cases, the embedded solution is slightly less accurate than the original diffusion solution with the errors introduced by the embedded method less than half a percent in all figures of merit for the power shape.

\section{MOX 1 Core}

The MOX cores have larger errors in the pin power distribution and the impact of the embedded method is more apparent. . Similar to the LEU core, the peak pin power is in the center assembly and embedded regions are gradually introduced to improve the solution. Figure 5.3 shows the error in the pin power distribution for the diffusion solution and the three multiscale cases. Although the peak pin power error increases slightly, the embedded method reduces the maximum pin power error and the global RMS error. The error in the pin power shape is also decreased. As the multiscale regions are increased the pin power errors in the center assembly become constant. This is because the transport effects at the MOX/LEU interface are not completely resolved. A fourth multiscale case is rerun where the MOC solver is used in the center two assemblies. The seven group pin homogenized diffusion solver is also used in the third assembly. Figure 5.4 shows the multiscale levels used in the additional calculation.

The impact of the extension of the MOC region is signiffcant. All of the figures of merit increase except the speedup as shown in table 5.2. Figure 5.5 contrast the original diffusion solution errors with the new multiscale solution. The interface effects are completely removed between the first two assemblies and are considerably reduced between the second and third assemblies. 


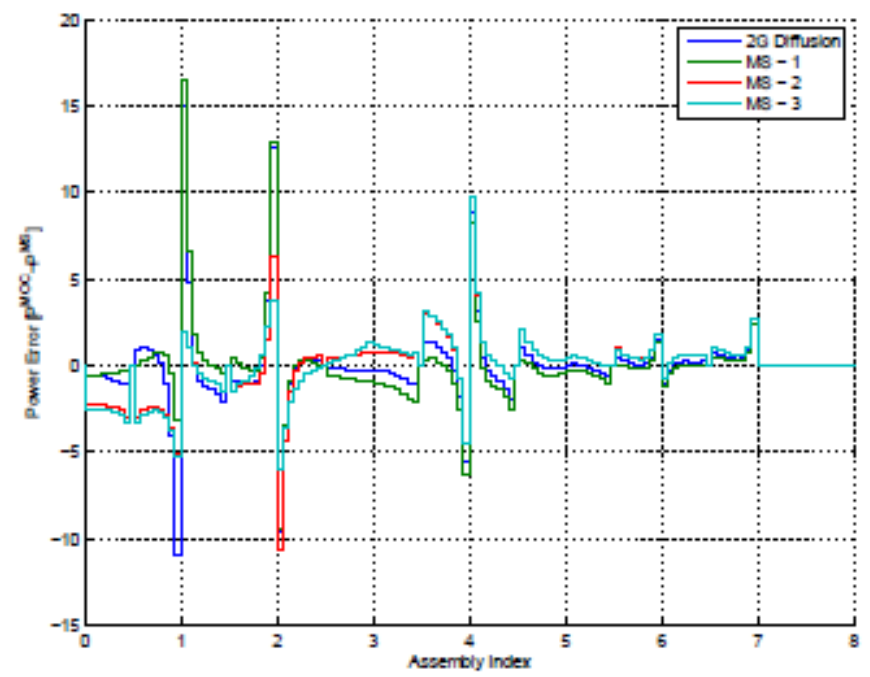

Figure 5.3: PPR in MOX1 Core for Embedded Method

\begin{tabular}{|l|l|l|l|l|l|l|}
\hline & & & & & & \\
\hline
\end{tabular}

\begin{tabular}{|l|l|l|}
\hline $76 \mathrm{MOC}$ & $7 \mathrm{G}$ Pin Diffusion & $4 \mathrm{G}$ Pin Diffusion \\
\hline
\end{tabular}

Figure 5.4: Additional Embedded Multiscale Region for MOX 1 Core

Table 5.2: Figures of Merit for MOX1 Core with Embedded Method

\begin{tabular}{|c|c|c|c|c|c|c|}
\hline & $\tau$ & $\Delta \mathrm{k}$ & $E^{\text {Panx }}$ & $E^{\min }$ & $E^{E^{n t}}$ & मालक्ष \\
\hline 2G Diflusion & 256.6 & -28.4 & $-1.07 \%$ & $15.03 \%$ & $2.66 \%$ & $1.47 \%$ \\
\hline & 5.5 & -84.2 & $-0.23 \%$ & $16.53 \%$ & $2.58 \%$ & $0.46 \%$ \\
\hline MS - 2 & 5.3 & 64.0 & $-2.99 \%$ & $10.68 \%$ & $2.11 \%$ & $0.51 \%$ \\
\hline MS - 3 & 6.4 & 53.6 & -3.3. & $9.77 \%$ & $1.94 \%$ & 0.4 \\
\hline IS- Extended & 40 & 1.4 & $-0.44 \%$ & $9.00 \%$ & $1.22 \%$ & $0.00 \%$ \\
\hline
\end{tabular}

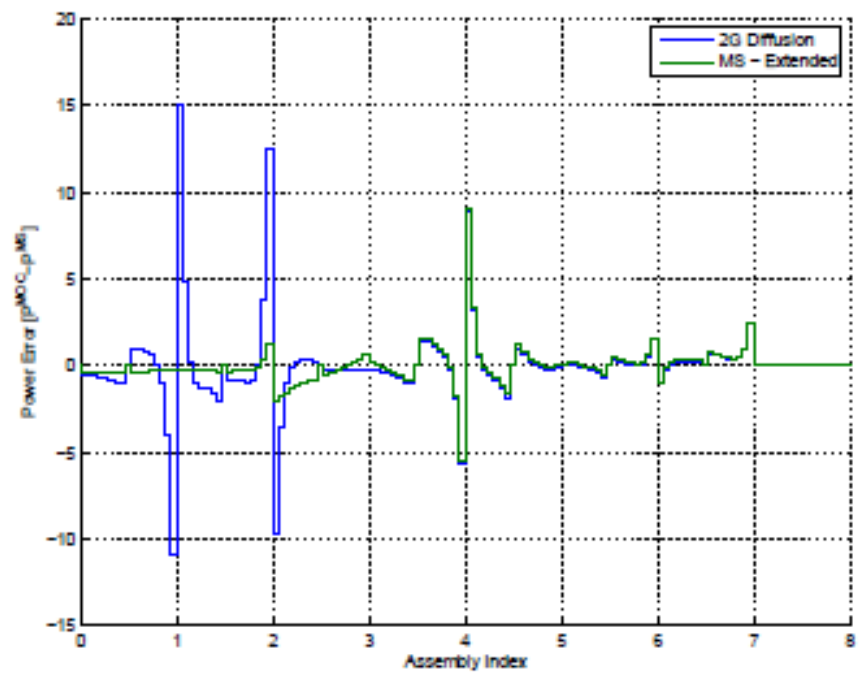

Figure 5.5: Impact of Extended MOC Region for MOX1 Core

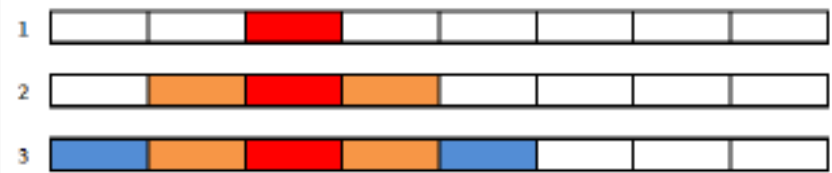

\begin{tabular}{|l|l|l|}
\hline $7 G ~ M O C$ & $7 G$ Pin Diffusion & 4G Pin Diffusion \\
\hline
\end{tabular} 
Figure 5.6: Multiscale Regions for MOX2 Core

Table 5.3: Figures of Merit for MOX 2 Core

\begin{tabular}{|c|c|c|c|c|c|c|}
\hline & 7 & $\Delta k$ & Epent: & $L^{\max }$ & $E^{\text {WhIs }}$ & $b^{\text {Troms nhiop }}$ \\
\hline 2G Diffusion & 191.6 & -36.3 & $-1.77 \%$ & $10.70 \%$ & $2.60 \%$ & $3.67 \%$ \\
\hline MS - 1 & 5.2 & -77.4 & $-1.58 \%$ & $11.71 \%$ & $2.66 \%$ & $3.70 \%$ \\
\hline MS - 2 & 4.6 & -2.0 & $-1.40 \%$ & $12.32 \%$ & $2.23 \%$ & $3.85 \%$ \\
\hline$M S-3$ & 5.0 & 34.5 & $-2.15 \%$ & $8.05 \%$ & $193 \%$ & $1.37 \%$ \\
\hline
\end{tabular}

MOX 2 Core

The MOX2 core requires the embedded region to be moved away from the center of the core as shown in Figure 5.6. The embedded method in this case decreases the RMS and shape errors but as shown in table 5.3 clear trends are not apparent in the peak pin error and the maximum overall error. The maximum error can be difficult to quantify because the location of the maximum pin error can change spatial location as the levels are expanded. It is not until the majority of the solution uses a higher level that the solution shows improvements in the maximum pin error. The principal issue with this case is that the energy structure never an asymptotic spectrum. The prolongation condition relies on the energy shape to be close to symmetric at an interface.

\section{Embedded Method with More Energy Groups}

The embedded method was then extended to more energy groups with the 33 group transport library used to assess the impact of using more energy groups. The 33 group MOC solver is embedded in the assembly with the peak power. Two different levels are examined to determine how important the energy group structure is around the MOC region. The first is a series of 33 group and 7 group pin homogenized diffusion solvers around the MOC region. The second is series of 7 group and 4 group pin homogenized diffusion solvers around the MOC region. Figure 5.7 shows the multiscale regions for two cases using the LEU and MOX 1 cores. All three cores were considered with these regions and both level structures gave very similar answers in all cases. This suggests that the impact of the reduced energy groups used in the second level structure is still suffcient to capture the spectral effects caused by adding more groups to the transport library. Table 5.4 summarizes the comparison of the two level structures for all three cores.

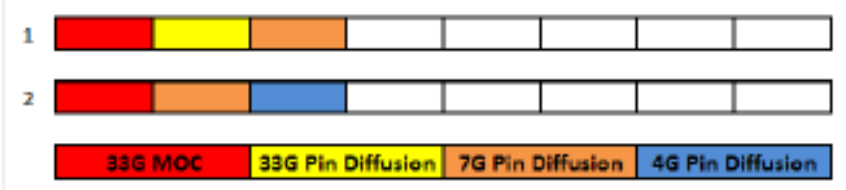

Figure 5.7: Multiscale Regions for 33 Group Embedded Calculation

Table 5.4: Comparison of Level Structure for 33 Group Embedded Calculation 


\begin{tabular}{|c|c|c|c|c|c|c|}
\hline & $\tau$ & $\Delta \mathrm{k}$ & $E^{\text {porak }}$ & $E^{\max }$ & $E^{\text {TOMS }}$ & $E^{\text {roms nape }}$ \\
\hline 2G Diffusion & 1220.6 & 48.4 & $-1.16 \%$ & $18.55 \%$ & $3.23 \%$ & $1.70 \%$ \\
\hline MS - 1 & - & 59.7 & $-3.83 \%$ & $11.45 \%$ & $2.14 \%$ & $0.69 \%$ \\
\hline MS - 2 & 6.8 & 86.8 & $-2.35 \%$ & $11.40 \%$ & $2.03 \%$ & $0.68 \%$ \\
\hline
\end{tabular}

Embedded Region Around Fuel-Reflector Interface

Adding the embedded region at the fuel reflector interface is another way that the embedded method can be used to increase the local accuracy and also the global accuracy. For all three cores, the MOC region is placed in the water reflector. Additional cases are added to model the last assembly using the seven group pin homogenized diffusion solver and the next assembly using the four group pin homogenized solver. Figure 5.8 shows the three multiscale regions considered. Although the LEU core already has a small pin power error, there is a noticeable tilt in the pin power error across the core. This is primarily because the leakage estimated at the fuel/reflector interface can have small errors that introduce a tilt in the flux and power distributions. Adding the multiscale region to the reflector will accurately model the correct effects at the boundary. Figure 5.9 shows the global shape of the pin power errors as the multiscale region is increased.

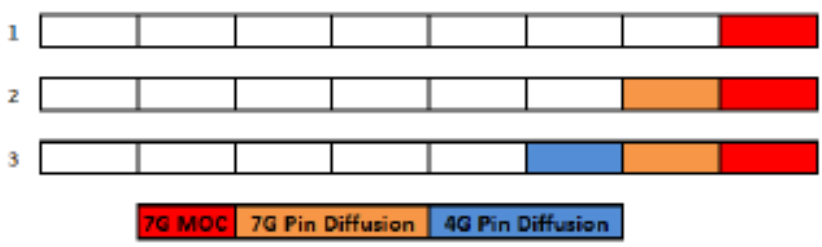

Figure 5.8: Multiscale Regions in the Reector for the Embedded Method

Although the pin power errors improve when the MOC region is added only in the reflector, it should be noted that the energy spectrum shape used for the prolongation operator at the core/reflector interface is very approximate since the core solution is uses 2 groups and the reflector uses 7 groups. Also, since the multiscale calculation does not have a direct impact on the fission source, the solution takes a long time to converge. Adding another multiscale region at the core reflector interface increases the accuracy of the calculation and actually decreases the run time because the energy levels of the core and reflector are better coupled.

For the MOX 1 core there is no improvement of the solution by adding multiscale regions in the reflector region. This is generally because the errors in the core leakage are overshadowed by the errors at the MOX/LEU interface. Some improvements are observed in the MOX 2 core as shown in Figure 5.10. Although the reductions in error are very modest, because the impact is overshadowed by the MOX/LEU interface, the principal improvement is the impact of calculating the correct discontinuity factor on the fuel/reflector interface. The reflector discontinuity factor was calculated using a two node problem with an LEU assembly and the reflector. Since the outgoing spectrum in LEU and MOX are different, the reflector discontinuity 
factor should be different for the two cases. It is not practical to generate a new discontinuity factor for every assembly facing the reflector so the common practice is to only generate one discontinuity factor for all assemblies at the periphery.

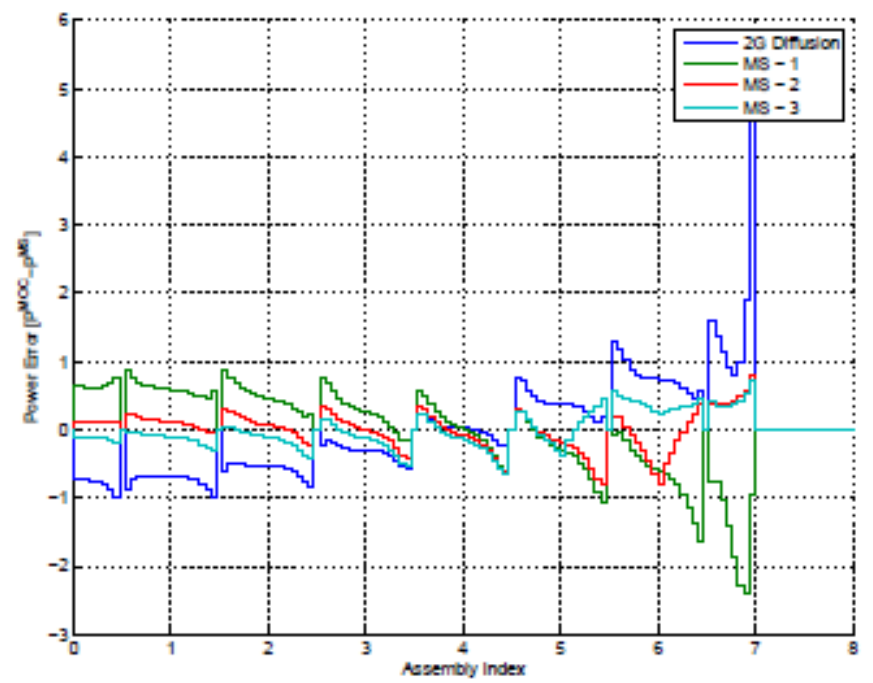

Figure 5.9: Impact of Embedded Regions in Reflector for the LEU Core

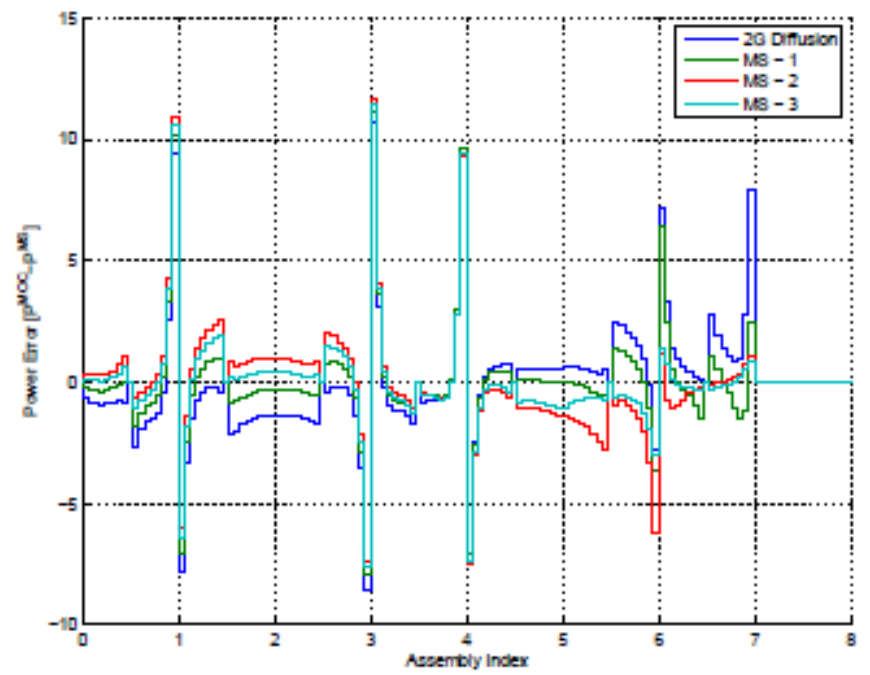

Figure 5.10: Impact of Embedded Regions in Reflector for the MOX 2 Core

\section{Embedded Region Around Controlled Assembly}

Although the change in reactivity due to a control rod (control rod worth) movement is very important during transient simulations, the ability to predict the power of the fuel pins around the control rod is also important to ensure when evaluating energy deposition limits. In order to test the ability to calculate both control rod worth and the pin power error around a control rod, the LEU core is used with a control rod inserted in the fourth assembly, as shown in Figure 5.11. 
The multiscale regions are set up in a consistent manner with the proceeding cases and can be seen in Figure 5.12.

The control rod worth is calculated using the rodded and unrodded eigenvalues as shown in equation (5.1).

$$
\rho_{\mathrm{CR}}=\frac{\mathrm{k}_{\text {Unrodded }}-\mathrm{k}_{\text {Rodded }}}{\mathrm{k}_{\text {Unrodded }} \mathrm{k}_{\text {Rodded }}}
$$

\begin{tabular}{|l|l|l|l|l|l|l|l|}
\hline LEU & LEU & LEU & LEU & Control & LEU & LEU & Refl \\
\hline
\end{tabular}

Figure 5.11: Control Insertion Pattern

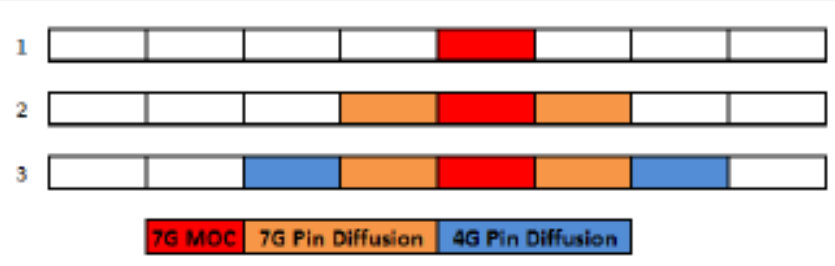

Figure 5.12: Multiscale Regions Used in Control Rod Analysis

For the LEU core all methods were able to accurately predict the worth of the control rod as shown in table 5.5. Although the diffusion solution was able to predict the global solution accurately, the local solution has significant errors as shown in Figure 5.13. PPR has the largest errors in pin power in the assembly with the control rod. The error in the PPR solution is caused by the modulation of the pin power distribution onto an assembly where the control rod has been homogenized. The impact of the embedded region reduces the local pin power errors and improves the global solution.

Table 5.5: Control Rod Worth for the LEU Core

\begin{tabular}{|r|c|c|}
\hline & $\rho C R$ & $\Delta \rho$ \\
\hline MOC & 357.3 & - \\
\hline 2G Diffusian & 358.8 & 1.5 \\
\hline MS - 1 & 341.8 & -15.5 \\
\hline MS - 2 & 345.1 & -12.2 \\
\hline MS - 3 & 347.2 & -10.1 \\
\hline
\end{tabular}




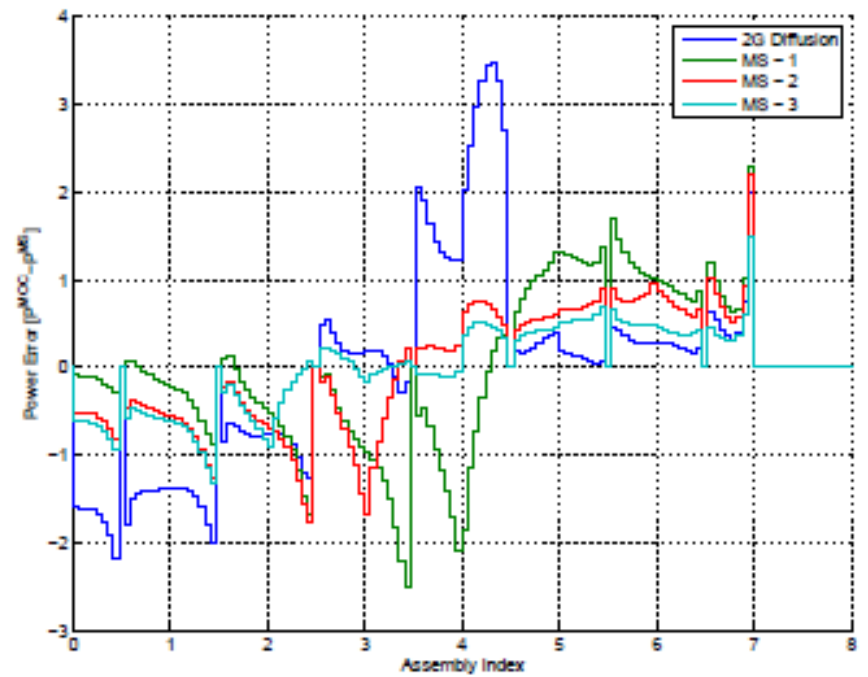

Figure 5.13: Pin Power Errors with Control Rod Inserted

Although the pin power errors occur at regions around the control rod where the power is low, the error can still be significant when considering an accident such as a control rod ejection. When the control rod is ejected from the core, a very localized power pulse occurs and the pins closest to the control rod can experience a significant energy deposition. The design limits of the reactor are evaluated in terms of the highest energy deposition in any one fuel pin after a control rod ejection.

\section{Discussion of Embedded Multiscale Method}

The embedded method evaluated in this chapter is more consistent with traditional multiscale methods used elsewhere in computational science and engineering than the post-refinement method discussed in Chapter 4. The ability to influence the global solution by locally refining the space, energy and angular mesh is a potentially powerful technique for improving the analysis of nuclear reactor performance. Several conclusions can be drawn from the results in this section to provide a set of best practice guidelines for implementing and further developing embedded methods with established computer codes such as PARCS and DeCART.

Impact of Multiple Embedded Levels

As noted in the post-refinement method discussion, the energy prolongation operators are much more significant than angle or space. The ability to use multiple embedded levels provides a solution to this problem without significantly reducing runtime. The use of a fine group pin homogenized diffusion solver surrounding the MOC region decreases the impact of the prolongation operator near the MOC region and improves the solution. The introduction of an intermediate group pin homogenized solver also decreases the impact of the energy prolongation operator. By decomposing the solution into three regions with different energies, the 
prolongation is a much smoother transition. The impact of introducing multiple levels is very similar to the buffer region used in the post-refinement method. Both the buffer region and adding multiple levels both attempt to reduce the impact of the boundary conditions of the higher scale solutions.

The analysis using 33 energy group library also showed that a fine group diffusion solver was not necessary to capture the energy effects and the 7 group diffusion solver was sufficient to describe the energy spectrum with a low level transport solution such as diffusion theory. This suggests that the need for a pin homogenized diffusion solver with the same number of energy groups as the transport solution is not necessary. Reducing the size of the requirements of the homogenized diffusion solver will reduce runtime and memory requirements for the multiscale methods.

\section{Prolongation Operators}

The prolongation operator for energy was chosen to reflect the results of the post-refinement method. The post-refinement method assessment concluded that the albedo type boundary conditions were much more stable. For this reason, the energy shape was chosen to use the outgoing current to define the shape of the incoming current.

Unfortunately there are cases where this type assumption is not valid. Higher order methods need to be implemented to make the embedded iteration method general enough to handle any arbitrary geometry. Methods such as the discrete generalized multigroup method could be used to better estimate the energy spectrum at the interface but have yet to be demonstrated in multidimensional cases.

\subsection{Summary}

In the research here, two different multiscale methods have been investigated which rely on a global diffusion solution to provide boundary conditions for a local region of interest. The local region is solved using a higher order method which for the work here was chosen to be an integral transport MOC solver. In the post-refinement multiscale methods the boundary conditions are transferred in one direction from the low order local solution to the high order global solution and the local solution does not attempt to correct the global solution. Two methods were investigated to implement the post-refinement method which employs different solution procedures. The first is a fixed source problem where the flux distribution is solved for a given incoming angular flux at the boundary. The second method provides an albedo boundary condition and solves a local eigenvalue problem.

The post-refinement methods were assessed using a series of one dimensional core models with a mixture of LEU and MOX assemblies. Cases were analyzed where the global and 
local solutions had the same energy group structure to analyze the effect of angular prolongation. Then mixed energy cases were solved where more energy groups were used in the local solution to test the energy prolongation operations.

For the cases with the same energy group structure, all methods performed very well and showed improvement over the standard methodology of diffusion with pin power reconstruction. The mixed energy cases exposed some problems with the energy prolongation operators. The albedo method showed improvement over the state of the art method for most cases considered but the fixed source method only showed better results when a buffer region was included. After analyzing the two different methods it was determined that the albedo method was more robust and consistent at improving the estimations of the peak pin power and the shape of the pin power distribution.

Therefore the albedo method was implemented into a two dimensional multiscale code system. Both the LEU core and the MOX cores showed improved estimations of the local pin powers for the cases run.

The second multi-scale method investigated in the research here was the embedded method which is more consistent with multi-scale methods currently used in computational science and engineering in which refinements in the local solution impact the global solution. The embedded method implemented here was assessed using the same one dimensional core models used in the post refinement method. Cases were examined where the embedded region is placed in the peak power assembly, at the fuel/reflector interface, and around a control rod. The embedded method showed the ability to reduce the global RMS pin power errors in almost every case analyzed, however the impact on the peak pin power shape was not uniform. There were several cases where the impact on the peak pin power did not change or errors even slightly increased. This was determined to be an impact on the errors in the net currents imposed on the boundary. Embedding the local solver into the reflector region demonstrated the ability to more accurately model the leakage effects out of the reactor and significantly decreased the tilt in the error across the LEU core. The MOX cores showed little or no improvement in the global errors when the local solver was embedded into the reflector. This is primarily because the MOX/LEU interface errors overshadowed any impact of improvements in describing the core leakage. The last case considered was the impact of embedding the local solver around a control rod. No noticeable improvements were observed in the determination of the control rod worth, however improvements were observed in the accuracy of local pin power predictions which is important during core safety analysis. Throughout the series of cases considered in this work several observations were made. For the post-refinement methods it was observed that the pin power accuracy can only be as accurate as the global power distribution in the region of interest. If the global assembly power has large errors, the pin powers will have similar errors. The numerical stability of the fixed source and albedo methods were assessed by evaluating the impact of small errors introduced in the boundary conditions. Numerical tests of both methods showed that the 
albedo method is much less sensitive to errors in the boundary condition. For the embedded method, it was observed that the inclusion of several solution levels increases the accuracy of the method. Specifically, the addition of multigroup pin homogenized diffusion solvers around the region of interest can decrease both local and global errors. 


\section{Chapter 3 Multiphysics Code for Implementation of Multiscale Methods}

This Chapter will describe the mutliphysics core analysis code AGREE/PARCS developed for implementation of the Multiscale Methods. In the following section, the theory and methods of the HTR gas dynamics and heat transfer code are described. In the subsequent section the neutronics nodal method TRIPEN is presented which provides the full core neutronics and power distribution.

\subsection{HTR Fluids Model in AGREE}

After reviewing the necessary physics required for PMR core fluids modeling, a set of code requirements has been established for the fluid flow module. The key requirements are: calculation of core bypass flow, capturing the momentum flux term in the momentum equation, and the ability to handle flow reversals. A Phenomena Identification and Ranking Table (PIRT) process has been completed for the NGNP [2]. The accident and thermal fluids PIRT panel has identified core coolant bypass flow as having an importance rank of high with a corresponding knowledge level of low. In the case of the PMR with the inter-block gaps, radial and axial manufacturing and refueling tolerances for the graphite blocks, irradiation swelling and cracking and the distribution of the thermal expansion, past experience and expertise indicates that the core bypass flow could be a significant proportion of the total core flow [3]. The bypass flow paths are located in the in-core and ex-core vertical columns between the stacks of hexagonal graphite blocks and through control rod holes within the graphite blocks, see Fig. 1.3 and Fig. 1.4. Additionally, the coolant can pass between the primary coolant flow path and the bypass flow path through horizontal gaps formed between the stacked graphite blocks. Therefore, the core fluid flow is essentially three-dimensional.

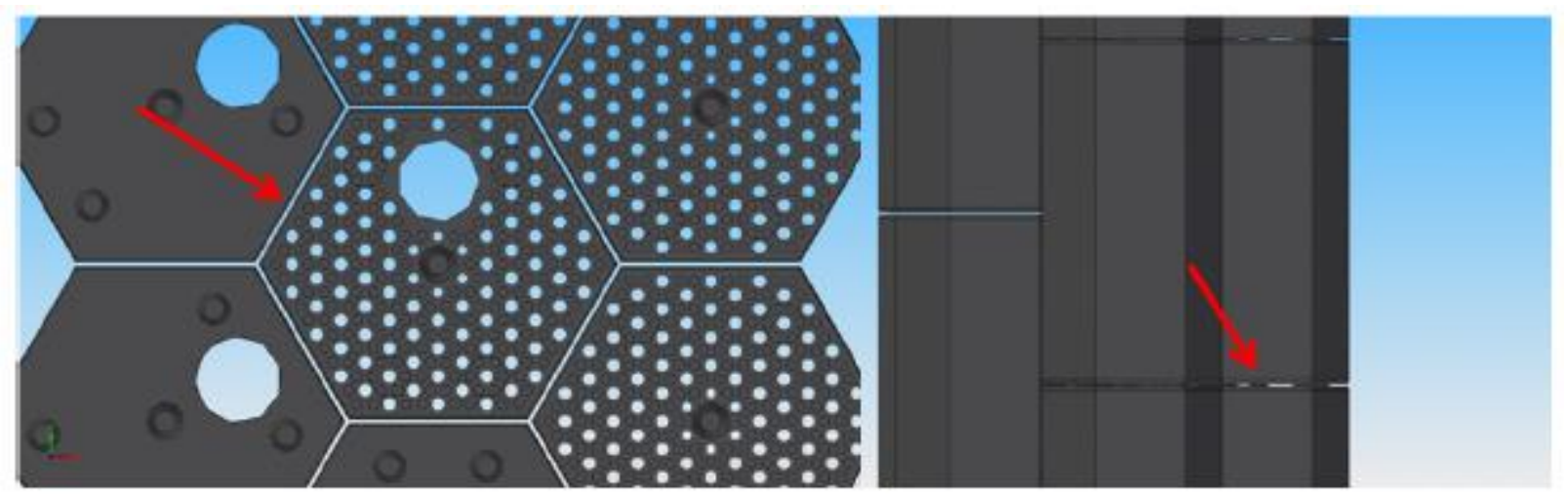

Figure 1.3: Location of bypass flowpaths in the NGNP 


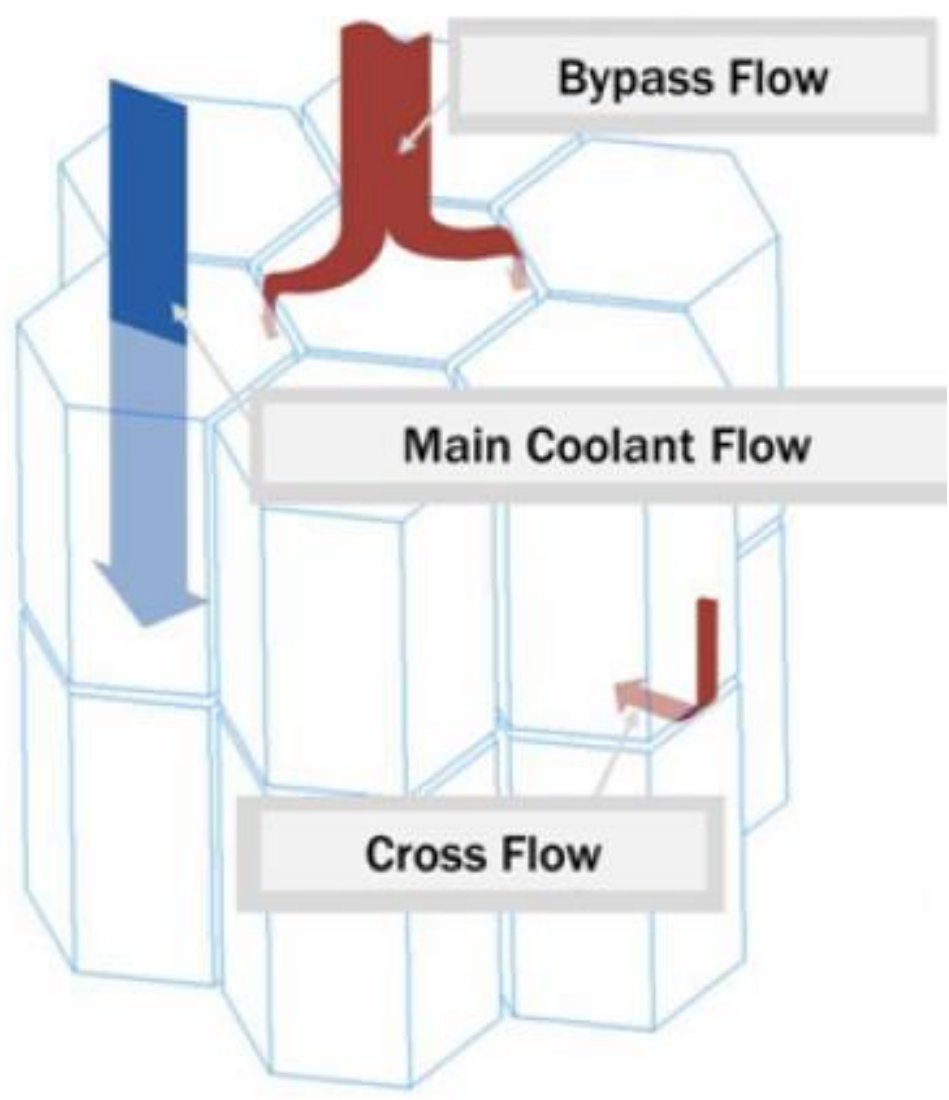

Figure 1.4: Bypass and crossflow paths in the NGNP

\section{Review of Existing Modeling Methods}

Several approaches to modeling fluid flow though the core of a PMR have been utilized that range from flow networks to full 3D CFD simulation. Flow network codes such as FLOPSY [4] and GAS-NET [5], shown in Fig 1.5, have been used by General Atomics and others to determine the flow distribution in the PMR core. These flow network codes are zero-dimensional in the sense that the convective terms are neglected and the solution represents a balance between pressure and friction forces. Neglecting the convective terms makes the solution computationally inexpensive, but these terms can be significant, especially during a transient (e.g. pressure wave propagation, flow-reversal, etc.). 


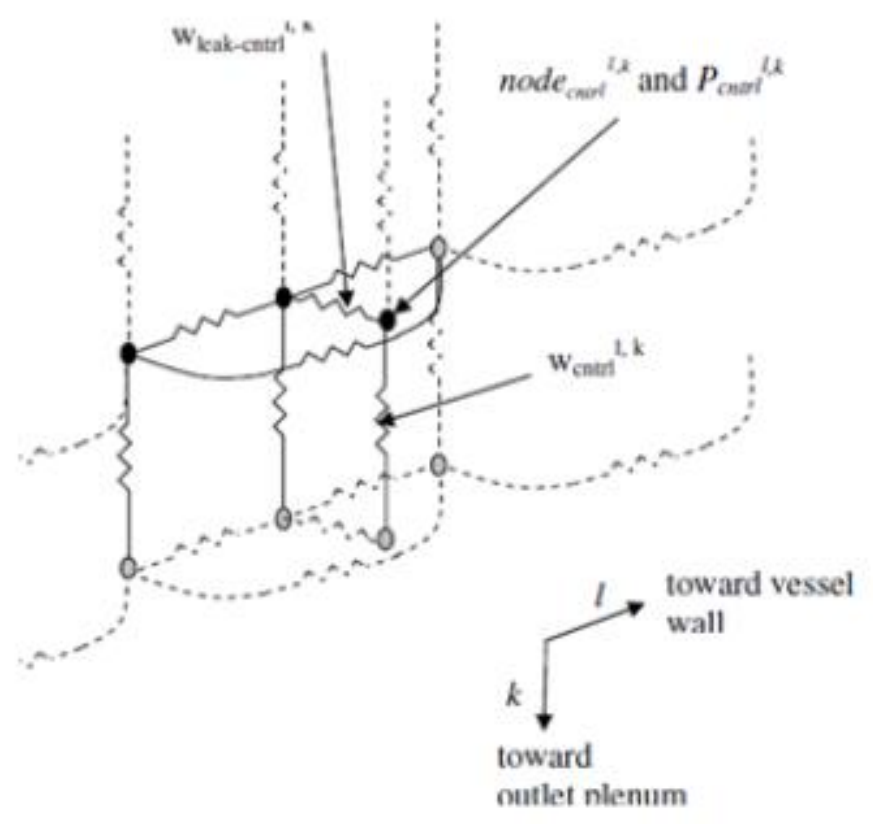

Figure 1.5: Representative computational domain in GAS-NET (figure taken from ANL-GenIV$071[3])$

Another approach is to use existing 1D system codes such as RELAP, TRACE, etc. (i.e. RELAP5 has been utilized to study leakage flows through the PMR core [6]). 1D system codes have the benefit of a very flexible input structure and a history of successful use for the analysis of light water reactors. These analyses typically utilize a nodalization that is quite coarse, see Fig. 1.6. Implementation of 1D system codes for PMR analyses may require substantial modification to implement the necessary physics and correlations, and concern remains in regards to 3D flow modeling. Finally, literature is available that demonstrates 3D CFD modeling of the PMR [7, 8]. CFD provides high fidelity solutions and has been coupled with neutron transport codes [8]. The primary drawback to CFD analysis is the large computational expense associated with the relatively finely meshed computational domain. This large computational expense is amplified by the length of the transients that need to be performed for the PMR (i.e. peak kernel temperature for the DLOFC occurs days into the event). As a compromise between the 1D system codes and CFD, a subchannel approach has been selected as the basis to model PMR fluids in AGREE. 


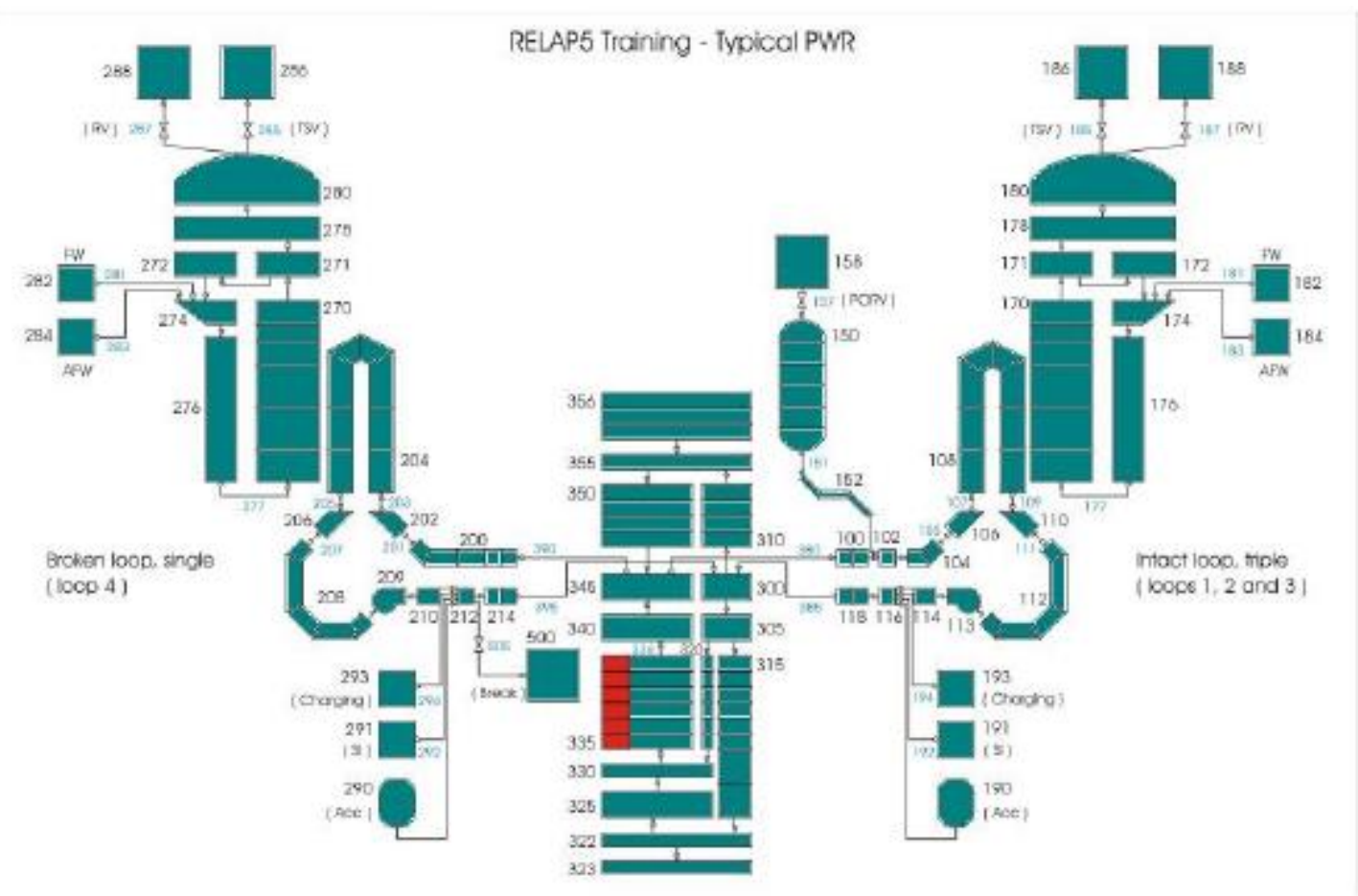

Figure 1.6: Typical nodalization used in a RELAP5 analysis for a pressurized water reactor.

\section{The Subchannel Approach}

The subchannel approach divides the entire core into several subchannels, where each subchannel is representative of the flow in that specified region of the core. Each subchannel consists of several smaller volumes stacked axially upon each other. These subchannels can be connected to other subchannels in juxtaposition by specifying gaps. Gaps consist of several junctions that provide a fowpath between adjacent subchannels. Calculating the axial momentum flux terms allows the code to capture the pressure and velocity changes that occur when flow acceleration is significant. This local acceleration can occur by area changes in the flowpath as well as expansion of the coolant due to heat transfer. Inclusion of the momentum flux terms results in non-linear partial differential equations which will be handled in the mathematical model. The normal flow path of the coolant through the core is from the top of the core to the bottom of the core. In the Loss of Forced Circulation (LOFC) accident scenario natural circulation is anticipated to occur during the transient, where the coolant flows from the bottom of the core to the top of the core. Hence, during the LOFC event the coolant flow through the core will initially be relatively large in the normal direction, slow down to stagnation and then change direction. In order for the code to capture this change in flow direction, a hybrid differencing scheme will be used in the axial dimension. The hybrid differencing scheme is a combination of upwind differencing with zero diffusion and central differencing [9]. 
The application of the subchannel approach to AGREE produces a three-dimensional core represented by a series of cross-connected one-dimensional subchannels. As a visualization aid, a cluster of six graphite-elements is shown in Fig. 1.7 and Fig. 1.8, where each graphite-element is broken up into six individual subchannels and each bypass flowpath is represented as an individual subchannel.

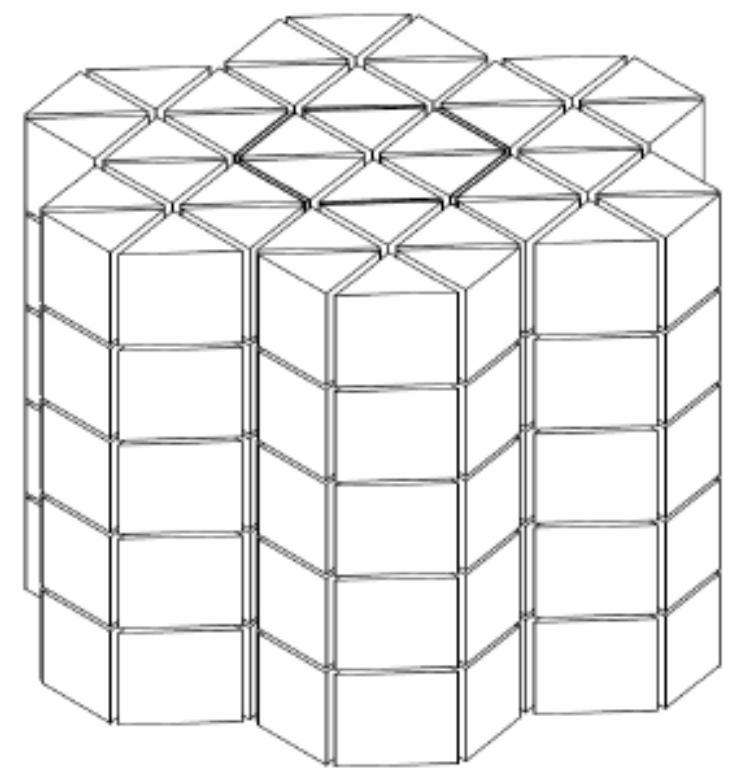

Figure 1.7: Representative computational domain used in the subchannel approach.

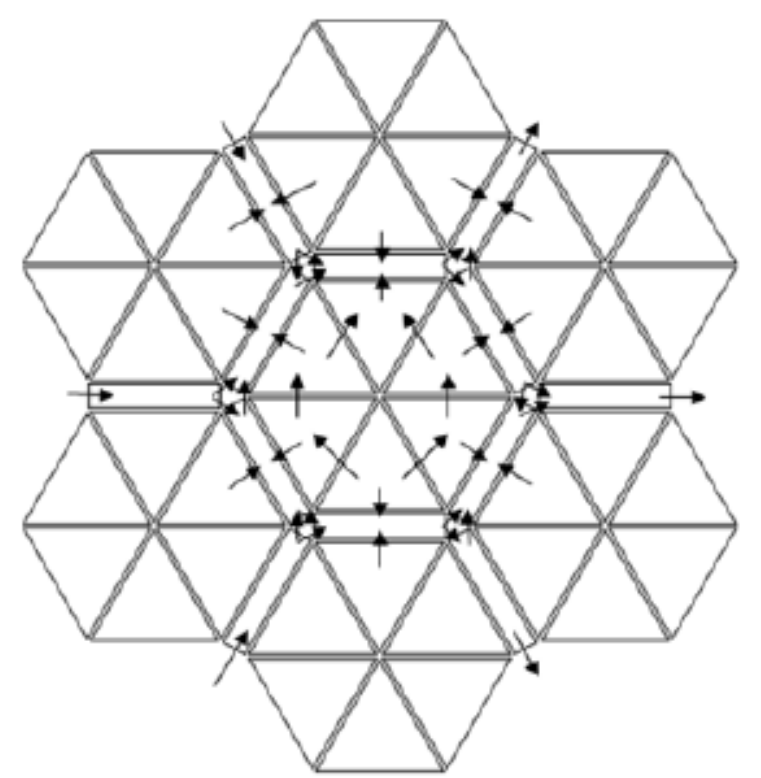

Figure 1.8: Top view of computational domain with emphasis on lateral flow junctions (gaps).

The velocity field is broken up into axial and lateral components. The axial component corresponds to the fluid owing vertically through the core and utilizes a general momentum 
transport equation. The lateral component of momentum is aligned normal to the axial component, and an assumption is imposed that the lateral component of fluid flow is only significant near the lateral faces. Hence, the lateral flux terms are assumed to be negligible. The assumption imposed on the lateral momentum equation has its origins in the subchannel codes used successfully for Light Water Reactor (LWR) core thermal-hydraulic analysis, namely the COBRA [11] and VIPRE codes [12]. This assumption greatly simplifies the governing equations by reducing a fully-three-dimensional system to a network of 1-D subchannels and has been applied successfully for LWR analysis where the axial component of the flow is significantly larger than the lateral component of the flow.

\section{Treatment at Block Interfaces}

The portrayal of lateral flow junctions shown in Fig. 1.8 demonstrates the radial locations of the junctions, but the axial locations of the junctions depend on what is being connected. The lateral junctions that connect bypass channels to adjacent bypass channels are placed at the lateral faces of the control volumes since these gaps are open along the entire length of the graphite block, and therefore do not impose any additional modelling concerns. Crossflow to or from the main coolant occurs at block faces, and therefore occurs at an axial face of a control volume and requires special treatment. Instead of placing a lateral junction at the top or bottom control volume within a graphite block, zero-volume nodes are placed between axially aligned components as shown in Fig. 1.9 and Fig. 1.10.

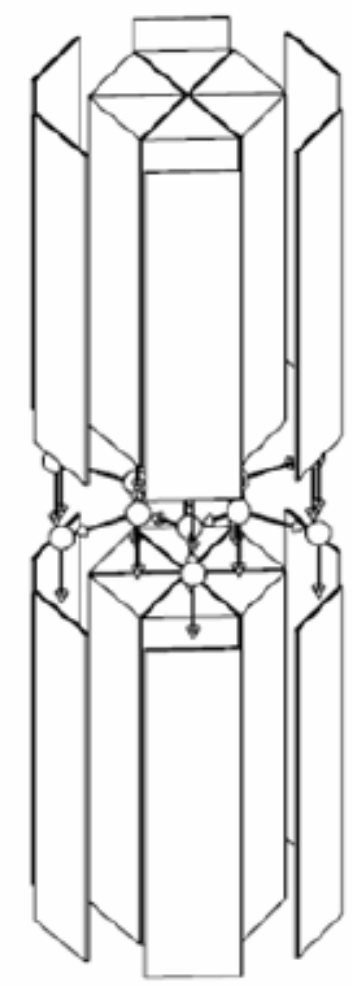

Figure 1.9: Illustration of axial components connected through zero-volume nodes. 


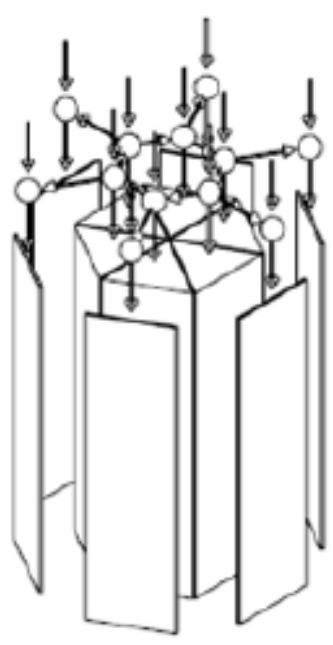

Isometric View

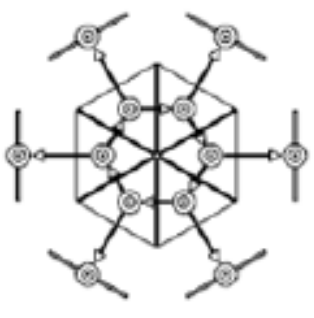

Top View

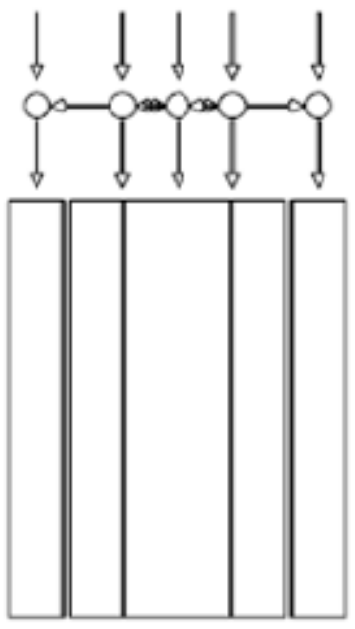

Front View

Figure 1.10: Lateral junctions connecting zero volume nodes for crossflow between coolant channels and between coolant channel and bypass channel.

\section{Mathematical Model}

Development of the mathematical model occurs in three stages: derivation of the general integral transport equation, application of the integral transport equation to obtain the appropriate balance equations, and discretization of the balance equations to obtain a form amenable to a linear solver. In the paragraph below, a precaution is given to the reader regarding the additional complexity added by the introduction of single-volumes and single-junctions into the model.

The computational domain of the mathematical model is represented by subchannels, singlevolumes, and single-junctions. A subchannel consists of user specified n volumes with $\mathrm{n} \square 1$ internal junctions. Therefore, the internal volumes inside of a subchannel have one inlet junction and one outlet junction, but the first and last volume inside of a subchannel can have an arbitrary number of inlet/outlet single- junctions. Likewise a single-volume can have an arbitrary number of inlet/outlet single-junctions. Hence, the introduction of single-junctions affect the convective term in the balance equations. During the conceptual development of AGREE, this added complexity was dealt with by deriving the appropriate discretizations for each situation that could be encountered [10]. After running actual test cases, it was found that the situations where these complexities arise are always associated with abrupt area changes and required special treatement. This speacial treatment is discussed in Section 1.3.2

\section{The Field Equations}

The field equations are obtained by applying the a generalized integral transport equation to the mass, axial momentum, and energy. The lateral momentum equation is obtained via a local 
instantaneous force balance. The procedure to obtain the general integral transport equation is widely available in the literature, and the procedure applied here is taken from Ishii [11]. A general integral balance is obtained by introducing the density $\rho$, the effux J, and the volumetric source $S_{\Phi}$ for any variable _.

$$
\frac{d}{d t} \int_{C V} \rho \phi d V=-\oint_{C S} \vec{n} \cdot \mathbb{J} d A+\int_{C V} S_{\phi} d V
$$

Application of the fundamental theorem of calculus to the time derivative results in the following:

$$
\frac{d}{d t} \int_{C V} \rho \phi d V=\frac{\partial}{\partial t} \int_{C V} \rho \phi d V+\oint_{C S} \rho \phi \vec{U} \cdot \vec{n} d A
$$

Combining these results, we obtain the general integral transport equation:

$$
\frac{\partial}{\partial t} \int_{C V} \rho \phi d V+\oint_{C S} \rho \phi \vec{U} \cdot \vec{n} d A=-\oint_{C S} \vec{n} \cdot \mathbb{J} d A+\int_{C V} S_{\phi} d V
$$

The integral transport equation can be reduced further to obtain the differential form of the transport equations. Since the code utilizes finite volumes, further reduction of the equations is unnecessary. Next, we apply the integral transport equation to a control volume, shown in Fig. 1.11, and carry out integrations over space and time. Integrating Eq. 1.3 over time:

$$
\begin{aligned}
\int_{C V} \int_{t}^{t+\Delta t} \frac{\partial \rho \phi}{\partial t} d t d V+\int_{t}^{t+\Delta t} \oint_{C S} \rho \phi \vec{U} \cdot \vec{n} d A d t= & -\int_{t}^{t+\Delta t} \oint_{C S} \vec{n} \cdot \mathbb{J} d A d t \\
& +\int_{t}^{t+\Delta t} \int_{C V} S_{\phi} d V d t
\end{aligned}
$$

For the representation of the term @__= @t, a single value for _ is assumed to prevail through the control volume. 


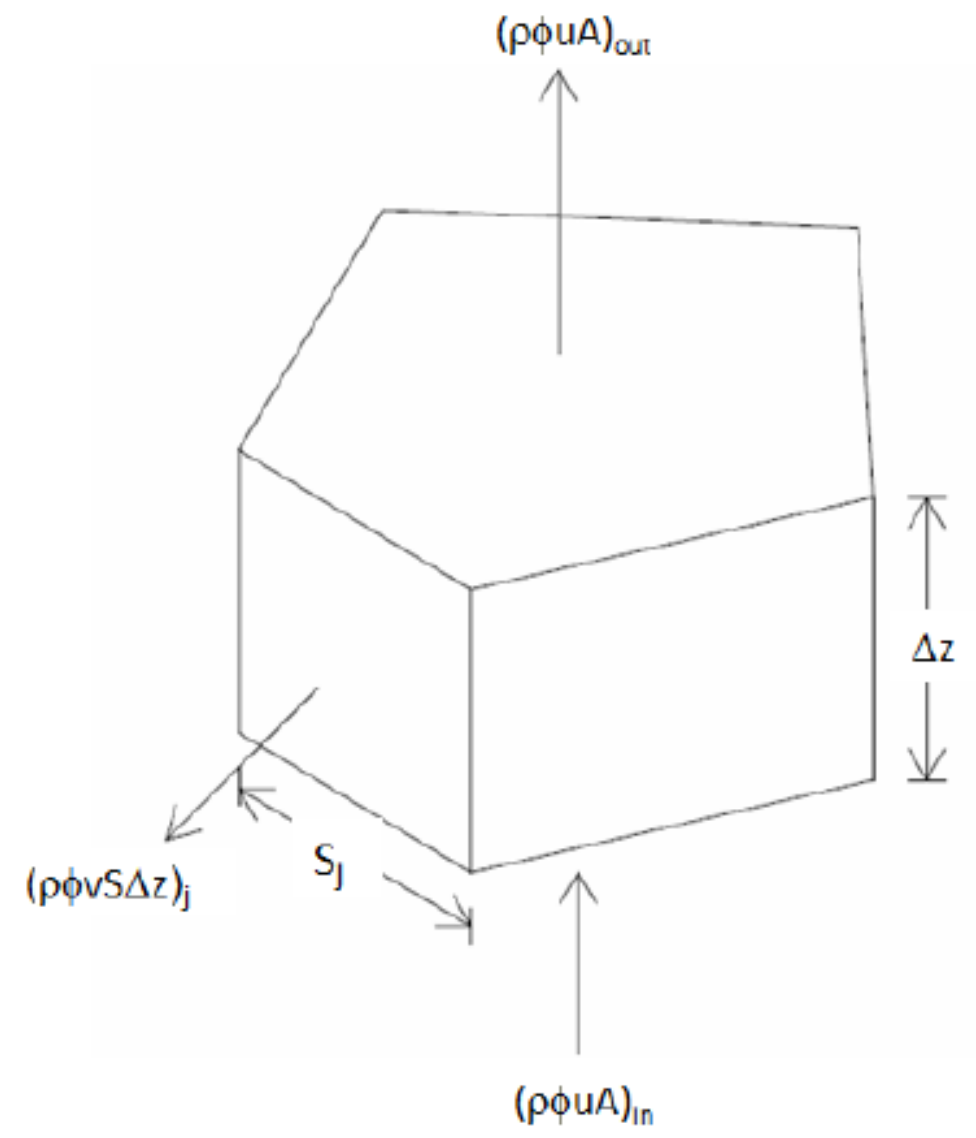

Figure 1.11: Representative control volume (shape is arbitrary) 


$$
\int_{C V} \int_{t}^{t+\Delta t} \frac{\partial \rho \phi}{\partial t} d t d V=A \Delta z\left[(\rho \phi)-(\rho \phi)^{0}\right]
$$

$$
\int_{t}^{t+\Delta t} \oint_{C S} \rho \phi \vec{U} \cdot \vec{n} d A d t=\Delta t\left[(\rho \phi u A)_{o u t}-(\rho \phi u A)_{\text {in }}+\sum_{j}(\rho \phi v S \Delta z)_{j}\right]
$$

$$
\int_{t}^{t+\Delta t} \oint_{C S} \vec{n} \cdot \mathbb{J} d A d t=\Delta t J_{o u t} A_{C S}
$$

$$
\int_{t}^{t+\Delta t} \int_{C V} S_{\phi} d V d t=A \Delta z \Delta t S_{\phi}
$$

$$
\begin{aligned}
\frac{A \Delta z}{\Delta t}(\rho \phi)+(\rho \phi u A)_{\text {out }} & -(\rho \phi u A)_{\text {in }}+\sum_{j}(\rho \phi v S \Delta z)_{j} \\
& =J_{\text {out }} A_{C S}+A \Delta z S_{\phi}+\frac{A \Delta z}{\Delta t}(\rho \phi)^{0}
\end{aligned}
$$

\section{Conservation of Mass}

Since there are no surface or volume sources of mass with respect to a fixed control mass, the efflux and volumetric source terms are zero. $\Phi=1, \mathrm{~J}=0, \mathrm{~S}_{\Phi}=0$ Hence, from Eq. 1.9 the continuity equation can be written as:

$$
\frac{A \Delta z}{\Delta t} \rho+\sum_{\text {out }}(\rho u A)_{\text {out }}-\sum_{\text {in }}(\rho u A)_{\text {in }}+\sum_{j}(\rho v S \Delta z)_{j}=\frac{A \Delta z}{\Delta t} \rho^{0}
$$

Eq. 1.10 is easily extended to a volume with multiple inlet/outlet junctions, see 
Eq. 1.11 .

$$
\frac{A \Delta z}{\Delta t} \rho+(\rho u A)_{o u t}-(\rho u A)_{i n}+\sum_{j}(\rho v S \Delta z)_{j}=\frac{A \Delta z}{\Delta t} \rho^{0}
$$

\section{Conservation of Energy}

An energy balance in term of enthalpy captures the work performed by the fluid across the boundaries of the control volume. The enthalpy transport equation is obtained by introducing the heat efflux and source terms into Eq. 1.9.

$$
\phi=h, \quad J_{\text {out }} A_{C S}=-q_{\text {in }}^{\prime \prime} \xi_{H} \Delta z-\left(k A \frac{\partial T}{\partial z}\right)_{\text {out }}+\left(k A \frac{\partial T}{\partial z}\right)_{\text {in }}, \quad S_{\phi}=q^{\prime \prime \prime} A \Delta z
$$

In the terms above, the viscous dissipation and the substantial derivative of pressure have been dropped as they have negligible contributions to the energy balance. The resulting enthalpy transport equation is given in Eq. 1.12.

$$
\begin{aligned}
\frac{A \Delta z}{\Delta t}(\rho h) & +(\rho h u A)_{\text {out }}-(\rho h u A)_{\text {in }}+\sum_{j}(\rho h v S \Delta z)_{j} \\
& =q_{\text {in }}^{\prime \prime} \xi_{H} \Delta z+\left(k A \frac{\partial T}{\partial z}\right)_{\text {out }}-\left(k A \frac{\partial T}{\partial z}\right)_{i n}+\frac{A \Delta z}{\Delta t}(\rho h)^{0}
\end{aligned}
$$

Eq. 1.12 is easily extended to a volume with multiple inlet/outlet junctions, see Eq. 1.13.

$$
\begin{aligned}
\frac{A \Delta z}{\Delta t}(\rho h) & +\sum_{\text {out }}(\rho h u A)_{\text {out }}-\sum_{\text {in }}(\rho h u A)_{\text {in }}+\sum_{j}(\rho h v S \Delta z)_{j} \\
& =q_{\text {in }}^{\prime \prime} \xi_{H} \Delta z+\sum_{\text {out }}\left(k A \frac{\partial T}{\partial z}\right)_{\text {out }}-\sum_{\text {in }}\left(k A \frac{\partial T}{\partial z}\right)_{\text {in }} \\
& +\frac{A \Delta z}{\Delta t}(\rho h)^{0}
\end{aligned}
$$




\section{Conservation of Axial Momentum}

The axial momentum equation is obtained by introducing the surface and body forces. Additionally, the axial momentum equations are solved at the faced of the control volume. Thus the pressures utilized in the axial momentum equation are taken at the centers of the adjacent control volumes. The efflux and source terms are given below.

$$
\phi=u, \quad J_{\text {out }} A_{C S}=\left(p_{\text {out }}-p_{\text {in }}\right) A+\left(K+\frac{f l}{D_{H}}\right)\left(\rho \frac{|u| u}{2} A\right), \quad S_{\phi}=(\rho A \Delta z) \cos \theta
$$

Placing the terms above into Eq. 1.9:

$$
\begin{aligned}
\frac{A \Delta z}{\Delta t}(\rho u) & +\left(\rho u^{2} A\right)_{\text {out }}-\left(\rho u^{2} A\right)_{\text {in }} \\
& =\left(p_{\text {in }}-p_{\text {ou }}\right) A-\left(K+\frac{f l}{D_{H}}\right)\left(\rho \frac{|u| u}{2} A\right) \\
& +(\rho A \Delta z) \cos \theta+\frac{A \Delta z}{\Delta t}(\rho u)^{0}
\end{aligned}
$$

Conservation of Lateral Momentum

The lateral momentum equations are obtained by performaning a localilzed in- stantaneous force balance on the lateral flow juncions. In the resulting equation, Eq. 1.15, the temporal and momentum flux terms drop out.

$$
K \rho \frac{|v| v}{2}=p_{\text {in }}-p_{\text {out }}
$$

Discretization of the Field Equations

In this section the transport equations are placed in a form amenable to a linear solver. The equations to be solved will be the energy equation, linear momentum equation, lateral momentum equation, and pressure correction equation. Although it may appear that the conservation of mass equation is not present, it will be shown to be present in the form of the pressure correction equation. Additionally, in the previous sections the flow across a boundary was specified as either in or out for simplicity. This simplified nomenclature will be insufficient in this section because different quantities are stored at different locations within the computational domain, i.e. fluid properties are stored at the centers of the control volumes, 
whereas the velocities are stored at the faces of the control volumes (junctions/gaps). Therefore, in and out will be replaced by iv, ov, ij, and oj for inlet volume, outlet volume, inlet junction, and outlet junction respectively.

\section{Useful Quantities}

From the previous paragraph, fluid properties are stored at the control volume centers and the velocities are stored at the faces of the control volumes in junctions and gaps. However, there are quantities that are required for nearly all the transport equations and need to be extrapolated. The calculation of density at the junctions and the mass flow at the volume center are useful quantities and are calculated below.

Junction density is obtained by linear interpolation:

$$
\begin{gathered}
\rho_{i j}=\frac{\rho \Delta z+(\rho \Delta z)_{i v}}{\left(\Delta z+\Delta z_{i v}\right)} \\
\rho_{o j}=\frac{\rho \Delta z+(\rho \Delta z)_{o v}}{\left(\Delta z+\Delta z_{o v}\right)}
\end{gathered}
$$

The mass flow rate calculated at the volume center utilizes the above definition and is applicable to a volume with $\mathrm{m}$ inlet junctions and $\mathrm{n}$ outlet junctions.

$$
F=\frac{1}{2}\left(\sum_{m}(\rho u A)_{o j}+\sum_{n}(\rho u A)_{i j}\right)
$$

\section{Conservation of Energy}

The conservation of energy equation is given by Eq. 1.12. To place this equation into an algebraic form, the differentials need to be approximated.

$$
\sum_{o u t} k A \frac{\partial T}{\partial z}=\sum_{o j} 2 k_{o j} A_{o j} \frac{T_{o v}-T}{\Delta z+\Delta z_{o v}}
$$


The equation above leads to the following definitions to simplify the expressions.

$$
\begin{gathered}
D_{h, o j}=\frac{2 k_{o j} A_{o j}}{\Delta z+\Delta z_{o v}} \\
D_{h, i j}=\frac{2 k_{i j} A_{i j}}{\Delta z+\Delta z_{i v}}
\end{gathered}
$$

Next, an approximation needs to be imposed for the enthalpy values that are convected though the faces. As a first approximation, and to aid in the development of the equations, the central differencing scheme will be applied.

$$
\begin{gathered}
h_{o j}=\frac{c_{p} T \Delta z+\left(c_{p} T \Delta z\right)_{o v}}{\Delta z+\Delta z_{o v}} \\
h_{i j}=\frac{c_{p} T \Delta z+\left(c_{p} T \Delta z\right)_{i v}}{\Delta z+\Delta z_{i v}}
\end{gathered}
$$

Then, the convective terms become the following:

$$
\begin{aligned}
\sum_{o u t}(\rho h u A) & =\sum_{o j}\left[(\rho u A)_{o j} \frac{\Delta z}{\Delta z+\Delta z_{o v}} c_{p} T\right] \\
& +\sum_{o j}\left[(\rho u A)_{o j} \frac{\Delta z}{\Delta z+\Delta z_{o v}}\left(c_{p} T\right)_{o v}\right]
\end{aligned}
$$

Eq. 1.24 leads to the following definitions to simplify the expressions. 


$$
F_{o j}=(\rho u A)_{o j}
$$

$$
F_{i j}=(\rho u A)_{i j}
$$

Using Eq. 1.19 through Eq. 1.26, conservation of of energy, Eq. 1.13, can be written in the discretized form of Eq. 1.27.

$$
\begin{aligned}
{\left[\frac{\rho A \Delta z}{\Delta t}\right.} & \left.+\sum_{o j}\left(D_{h, o j}+F_{o j} \frac{\Delta z}{\Delta z+\Delta z_{o v}}\right)+\sum_{i j}\left(D_{h, i j}-F_{i j} \frac{\Delta z}{\Delta z+\Delta z_{o v}}\right)\right] \\
& =\sum_{o j}\left(D_{h, o j}-F_{o j} \frac{\Delta z}{\Delta z+\Delta z_{o v}}\right) c_{p} T_{o v} \\
& +\sum_{i j}\left(D_{h, i j}+F_{i j} \frac{\Delta z}{\Delta z+\Delta z_{i v}}\right) c_{p} T_{i v} \\
& +q^{\prime \prime} \xi_{H} \Delta z-\sum_{j}\left(\rho c_{p} T v S \Delta z\right)_{j}+\frac{\rho A \Delta z}{\Delta t}\left(c_{p} T\right)^{0}
\end{aligned}
$$

For implementation, conservation of energy is expressed as Eq.1.28.

$$
a_{T} T=\sum_{o v} a_{T, o v} T_{o v}+\sum_{i v} a_{T, i v} T_{i v}+b_{T}
$$

with, 


$$
\begin{gathered}
a_{T, o v}=\left(D_{h, o j}-F_{o j} \frac{\Delta z}{\Delta z+\Delta z_{o v}}\right) c_{p, o v} \\
a_{T, i v}=\left(D_{h, i j}+F_{i j} \frac{\Delta z}{\Delta z+\Delta z_{i v}}\right) c_{p, i v} \\
a_{T}=\left[\frac{\rho A \Delta x}{\Delta t}+\sum_{o v}\left(\frac{a_{T, o v}}{c_{p, o v}}+F_{o j}\right)+\sum_{i v}\left(\frac{a_{T, i v}}{c_{p, i v}}-F_{i j}\right)\right] c_{p} \\
b_{T}=q^{\prime \prime} \xi_{H} \Delta z-\sum_{j}\left(\rho c_{p} T v S \Delta z\right)_{j}+\frac{\rho A \Delta z}{\Delta t}\left(c_{p} T\right)^{0}
\end{gathered}
$$

It must be stated again that the above discretization is for central differencing, and has one large drawback. Namely, central differencing can become unstable for large flow rates. This can be seen by understanding that all coefficients, given by Eq. 1.29, must be positive to ensure numerical stability. For this condition to be satisfied, the following criterion must be satisfied for central differencing to be stable.

$$
P e_{o j}<\frac{\Delta z+\Delta z_{o v}}{\Delta z_{o v}}
$$

Where Peoj $=$ Foj=Doj is the volume Peclet number and represents the relative ratio of convection to diffusion. As most of the flows this code is being design to analyze are strongly convective, the fine mesh size required to achieve stability with central differencing is not acceptable. A better approach is to use hybrid differencing, which is a combination of central differencing and zero diffusion upwind differencing [9]. The application of hybrid differencing at this stage is a simple matter. Eq. 1.28 remains unchanged. Only the coefficients in Eq. 1.29 need to be updated to reflect the hybrid differencing scheme as shown below. 


$$
\begin{gathered}
a_{T, o v}=\max \left\{0, D_{h, o j}-F_{o j} \frac{\Delta z}{\Delta z+\Delta z_{o v}},-F_{o j}\right\} \\
a_{T, i v}=\max \left\{F_{i j}, D_{h, i j}+F_{i j} \frac{\Delta z}{\Delta z+\Delta z_{o v}}, 0\right\} c_{p, i v} \\
a_{T}=\left[\frac{\rho A \Delta x}{\Delta t}+\sum_{o v}\left(\frac{a_{T, o v}}{c_{p, o v}}+F_{o j}\right)+\sum_{i v}\left(\frac{a_{T, i v}}{c_{p, i v}}-F_{i j}\right)\right] c_{p} \\
b_{T}=q^{\prime \prime} \xi_{H} \Delta z-\sum_{j}\left(\rho c_{p} T v S \Delta z\right)_{j}+\frac{\rho A \Delta z}{\Delta t}\left(c_{p} T\right)^{0}
\end{gathered}
$$

\section{Conservation of Axial Momentum}

Treatment of the axial momentum equations follows the same approach as the energy equation, but there is a notable difference. There is no axial diffusion term so central differencing is unconditionally unstable for axial momentum. Thus, equations are derived for upwind differencing. The conservation of axial momentum equation for a typical junction is given by Eq. 1.14. The algebraic approximation of this equation for flow in the normal direction is given by Eq. 1.32.

$$
\begin{aligned}
{\left[\frac{\rho A \Delta z}{\Delta t}\right.} & \left.+F_{o v}+\left(K+\frac{f l}{D_{H}}\right)\left(\rho \frac{|u|}{2} A\right)\right] u \\
& =F_{i v} u_{i j}+\left(p_{i v}-p_{o v}\right) A+\rho A \Delta z g \cos \theta+\frac{\rho A \Delta z}{\Delta t} u^{0}
\end{aligned}
$$

The algebraic approximation of Eq. 1.14 for flow in the reverse direction is given by Eq. 1.33. 


$$
\begin{aligned}
{\left[\frac{\rho A \Delta z}{\Delta t}\right.} & \left.-F_{i v}+\left(K+\frac{f l}{D_{H}}\right)\left(\rho \frac{|u|}{2} A\right)\right] u \\
& =-F_{o v} u_{o j}+\left(p_{i v}-p_{o v}\right) A+\rho A \Delta z g \cos \theta+\frac{\rho A \Delta z}{\Delta t} u^{0}
\end{aligned}
$$

Eq. 1.32 and Eq. 1.33 can be combined into a generalized upwind expression given by Eq. 1.34.

$$
\begin{gathered}
a_{u} u=a_{u, o j} u_{o j}+a_{u, i j} u_{i j}+\left(p_{i v}-p_{o v}\right) A+b_{u} \\
a_{u, o j}=\max \left\{0,-F_{o v}\right\} \\
a_{u, i j}=\max \left\{F_{i v}, 0\right\} \\
a_{u}=\frac{\rho A \Delta z}{\Delta t}+a_{u, o j}+a_{u, i j}+\left(F_{o v}-F_{i v}\right)+\left(K+\frac{f l}{D_{H}}\right)\left(\rho \frac{|u|}{2} A\right) \\
b_{u}=\rho A \Delta z g \cos \theta+\frac{\rho A \Delta z}{\Delta t} u^{0}
\end{gathered}
$$

\section{Special Models}

In modeling the actual reactor core, several abrupt area changes occur. Typical upwind differencing of the momentum equation is not capable of capturing Bernoulli's equation in the presence of an abrupt area change. As shown in Appendix A, an additional source needs to be added on the right hand side in order to preserve stagnation pressure with an abrupt area change. For practical problems, this additional source hinders convergence. An alternative solution is to treat the abrupt area change as a localized instantaneous change that satisfies the steady-state energy balance equations. Such an approach is taken in RELAP5 [12], and is utilized in AGREE for treatment at abrupt area changes and at the edges of block faces. 


\section{Expansion/Contraction}

The special treatment is for a generalized contraction, consisting of multiple up- stream junctions, shown in Fig. 1.12, requires the definition of an effective upwind area. The effective upwind area of the junction is calculated as shown in Eq. 1.36, where $\mathrm{W}$ is the mass flow rate:

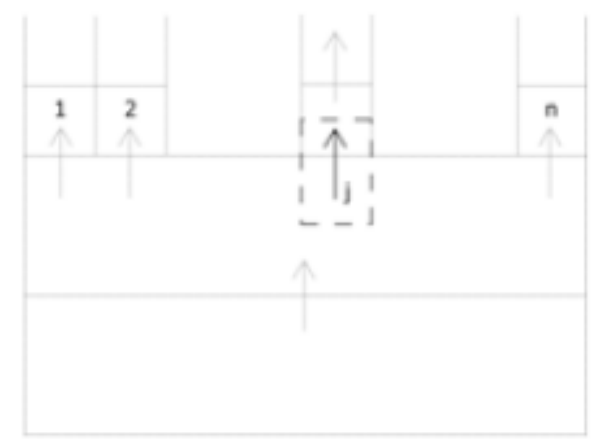

Figure 1.12: Visualization of a generalized contraction/expansion in AGREE

$$
A_{e f f}=\max \left\{A_{o v},\left|\frac{W_{j}}{W_{i v}}\right| A_{i v}\right\}
$$

Using the definition for Aeff, the discretization for the junction is derived from a mechanical energy balance:

$$
\left(p+\frac{1}{2} \rho u^{2}\right)_{i v}=\left(p+\frac{1}{2} \rho u^{2}\right)_{o v}
$$

Treating the fluid as incompressible, continuity gives Eq. 1.38, where $\epsilon=\mathrm{A}_{\mathrm{ov}} / \mathrm{A}_{\mathrm{eff}}$

$$
u_{i v}=u_{o v} \epsilon
$$

rearranging,

$$
p_{i v}-p_{o v}=\frac{1}{2}\left(1-\epsilon^{2}\right) \rho u_{o v}^{2}
$$

Introducing the additional loss and source terms, we arrive at the discretized equation given by Eq. 1.40 . 


$$
\begin{gathered}
a_{u}=\frac{1}{2}\left(1-\epsilon^{2}\right) W+\left(K+\frac{f l}{D_{H}}\right)\left(\rho \frac{|u|}{2} A\right) \\
b_{u}=\rho A \Delta z g \cos \theta
\end{gathered}
$$

The development for a generalized expansion is completely analogous. Further- more, the equations derived for the generalized contraction exactly reproduce the result for a generalized expansion when the flow is revered. Which is shown in the verification tests.

Expansion/Contraction with Crossflow

At block interfaces, the possibility of an abrupt area change with cross flow can occur. This situation is depicted in Fig. 1.13 and requires the introduction of three additional discretizations: junction type 10 which is located upstream of a zero volume node; junction type 11 which is located downstream from a zero volume node and associated with a contraction, and junction type 12 which is located downstream from a zero volume node and associated with a contraction

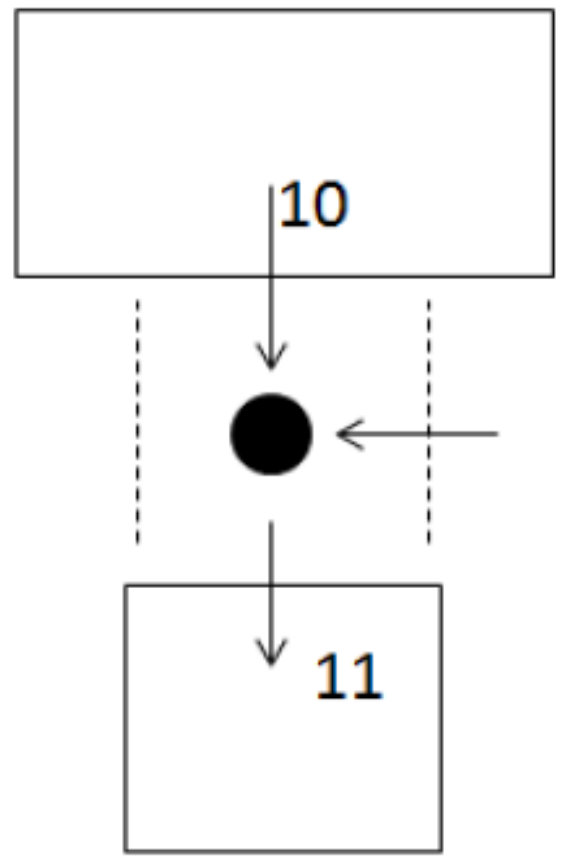

Contraction with Crossflow

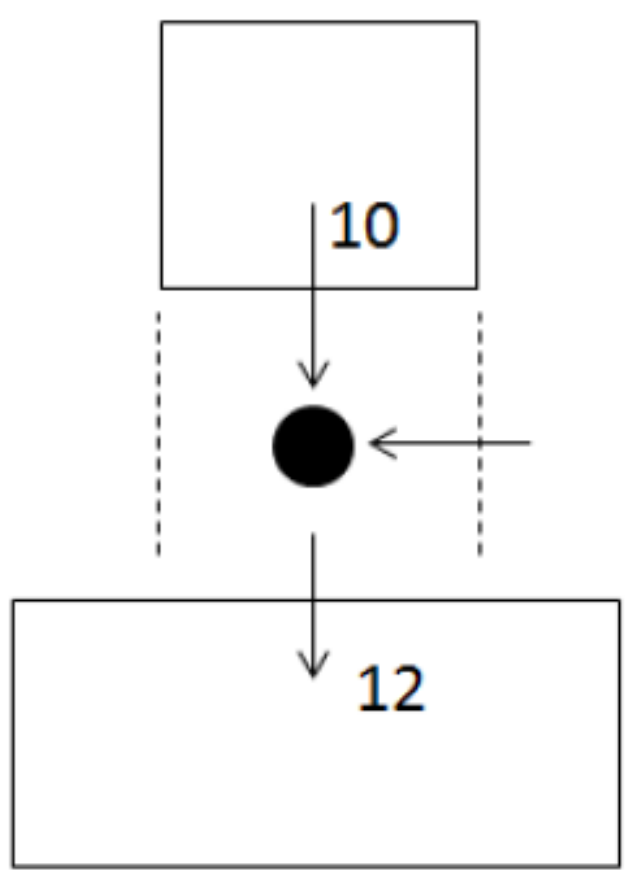

Expansion with Crossflow

Figure 1.13: Visualization of junctions at an abrupt area change with crossflow. 
Starting with the junction type 10/11 combination, the local instantaneous me- chanical energy balance in the forward direction gives:

$$
\begin{gathered}
p_{i v}-p_{\text {ov }}=\frac{1}{2}\left(1-\epsilon^{2}\right) \rho u_{10}^{2} \\
\left(p_{\text {iv }}-p_{\text {ov }}\right) A_{10}=\frac{1}{2}\left(1-\epsilon^{2}\right)\left(\rho u_{10} A_{10}\right) u_{10} \\
p_{\text {iv }}-p_{\text {ov }}=\frac{1}{2} \rho\left(u_{11}^{2}-u_{10}^{2}\right) \\
\left(p_{\text {iv }}-p_{\text {ov }}\right) A_{11}=\frac{1}{2}\left(\rho u_{11} A_{11}\right) u_{11}-\frac{1}{2}\left(\rho u_{10} A_{11}\right) u_{10} \\
=\frac{1}{2}\left(\rho u_{11} A_{11}\right) u_{11}-\frac{1}{2}\left(\rho u_{10} A_{10}\right) u_{10}
\end{gathered}
$$

The local instantaneous mechanical energy balance for the junction type 10/11 combination in the reverse direction gives:

$$
\begin{gathered}
p_{\text {ov }}-p_{\text {iv }}=\frac{1}{2} \rho \epsilon^{2} u_{10}^{2}-\frac{1}{2} \rho u_{11}^{2} \\
\left(-\frac{1}{2} \rho \epsilon^{2} u_{10} A_{10}\right) u_{10}=\left(-\frac{1}{2} \rho u_{11} A_{11}\right) u_{11}+\left(p_{i v}-p_{o v}\right) A_{10}
\end{gathered}
$$

For flow in the reverse direction, junction type 11 does see any expansion, contraction, or crossflow. Combining Eq. 1.43, Eq. 1.45, and Eq. 1.47, a generalized discretization can be obtained as in Eq. 1.48 for a junction of type 10, and Eq. 1.50 for a junction of type 11 .

$$
a_{u, 10} u_{10}=a_{u, 11} u_{11}+\left(p_{\text {iv }}-p_{\text {ov }}\right) A+b_{u}
$$


with,

$$
\begin{gathered}
a_{u, 10}=\max \left\{\frac{1}{2}\left(1-\epsilon^{2}\right) W,-\frac{1}{2} \epsilon^{2} W\right\}+\left(K+\frac{f l}{D_{H}}\right)\left(\rho \frac{|u|}{2} A\right) \\
a_{u, 11}=\max \left\{0,-\frac{1}{2} W_{11}\right\} \\
b_{u}=\rho A \Delta z g \cos \theta \\
a_{u, 11} u_{11}=a_{u, 10} u_{10}+\left(p_{\text {iv }}-p_{\text {ov }}\right) A+b_{u}
\end{gathered}
$$

Repeating this process with the junction type 10/12 combination results in the same discretization for junction type 10 and the discreatization for junction type 12 given by Eq. 1.52.

$$
a_{u, 12} u_{12}=a_{u, 10} u_{10}+\left(p_{i v}-p_{\text {ov }}\right) A+b_{u}
$$

With 


$$
\begin{gathered}
a_{u, 12}=\max \left\{\frac{1}{2} \epsilon^{2} W,-\frac{1}{2}\left(1-\epsilon^{2}\right) W\right\}+\left(K+\frac{f l}{D_{H}}\right)\left(\rho \frac{|u|}{2} A\right) \\
a_{u, 10}=\max \left\{\frac{1}{2} W_{10}, 0\right\} \\
b_{u}=\rho A \Delta z g \cos \theta
\end{gathered}
$$

\section{Pressure Correction Equation}

The pressure correction equation is obtained by introducing the momentum equations into the conservation of mass equation. The addition of special models to handle abrupt area changes and crossflows do not impact the form of the pressure correction equation. Therefore a single general pressure correction equation can be obtained which is applied to all volumes within the computational domain. The derivation of the pressure correction equation begins with a generalized conservation of mass equation and conservation of momentum equations.

$$
\begin{gathered}
\frac{A \Delta z}{\Delta t} \rho+\sum_{o j}(\rho u A)_{o j}-\sum_{i j}(\rho u A)_{i j}+\sum_{j}(\rho v S \Delta z)_{j}=\frac{A \Delta z}{\Delta t} \rho^{0} \\
a_{u} u=\sum_{o j} a_{u, o j} u_{o j}+\sum_{i j} a_{u, i j} u_{i j}+\left(p_{i v}-p_{o v}\right) A+b_{u} \\
a_{v} v=\left(p_{i v}-p_{o v}\right) A
\end{gathered}
$$

Next, split the pressure and velocities into a guess value and a correction value, as shown below where the starred quantities represent the guessed value. 


$$
\begin{aligned}
& p=p^{*}+p^{\prime} \\
& u=u^{*}+u^{\prime} \\
& v=v^{*}+v^{\prime}
\end{aligned}
$$

Place the pressure and axial velocity into the axial momentum equation.

$$
\begin{aligned}
a_{u}\left(u^{*}+u^{\prime}\right) & =\sum_{o j} a_{u, o j}\left(u_{o j}^{*}+u_{o j}^{\prime}\right)+\sum_{i j} a_{u, i j}\left(u_{i j}^{*}+u_{i j}^{\prime}\right) \\
& +\left[\left(p_{i v}^{*}+p_{i v}^{\prime}\right)-\left(p_{o v}^{*}+p_{o v}^{\prime}\right)\right] A+b_{u}
\end{aligned}
$$

The guessed velocity field satisfies the guessed pressure field with the corresponding source. Therefore, the velocity correction equation reduces to Eq. 1.59.

$$
a_{u} u^{\prime}=\sum_{o j} a_{u, o j} u_{o j}^{\prime}+\sum_{i j} a_{u, i j} u_{i j}^{\prime}+\left(p_{i v}^{\prime}-p_{o v}^{\prime}\right) A
$$

Next, the fundamental simplifying step of the SIMPLE algorithm is performed. The velocity correction terms in the inlet and outlet junctions are dropped.

$$
\begin{gathered}
a_{u} u^{\prime}=\left(p_{i v}^{\prime}-p_{o v}^{\prime}\right) A \\
u^{\prime}=d_{u}\left(p_{i v}^{\prime}-p_{o v}^{\prime}\right)
\end{gathered}
$$

Similary for the lateral momentum equation 


$$
v^{\prime}=d_{v}\left(p_{i v}^{\prime}-p_{o v}^{\prime}\right)
$$

The final step is to expand the conservation of mass equation in terms of the guessed and corrected values, and then replace the velocity correction terms with the pressure correction terms using Eq. 1.61 and Eq. 1.62. 


$$
\begin{aligned}
\frac{A \Delta z}{\Delta t} \rho & +\sum_{o j}(\rho A)_{o j}\left(u_{o j}^{*}+u_{o j}^{\prime}\right)-\sum_{i j}(\rho A)_{i j}\left(u_{i j}^{*}+u_{i j}^{\prime}\right) \\
& +\sum_{j}(\rho S \Delta z)\left(v_{j}^{*}+v_{j}^{\prime}\right)=\frac{A \Delta z}{\Delta t} \rho^{0}
\end{aligned}
$$

$$
\begin{aligned}
\sum_{o j}(\rho A)_{o j} u_{o j}^{\prime} & -\sum_{i j}(\rho A)_{i j} u_{i j}^{\prime}+\sum_{j}(\rho S \Delta z) v_{j}^{\prime} \\
& =\frac{\left(\rho^{0}-\rho\right) A \Delta z}{\Delta t}+\sum_{i j}(\rho A)_{i j} u_{i j}^{*} \\
& -\sum_{o j}(\rho A)_{o j} u_{o j}^{*}-\sum_{j}(\rho S \Delta z) v_{j}^{*}
\end{aligned}
$$

$$
\begin{aligned}
p^{\prime} \sum_{o j}(\rho A d)_{o j} & -\sum_{o j}(\rho A d)_{o j} p_{o v}^{\prime}-\sum_{i j}(\rho A d)_{i j} p_{i v}^{\prime}+p^{\prime} \sum_{i j}(\rho A d)_{i j} \\
& +p^{\prime} \sum_{j}(\rho S \Delta z)-\sum_{j}(\rho S \Delta z) p_{o v}^{\prime} \\
& =\frac{\left(\rho^{0}-\rho\right) A \Delta z}{\Delta t}+\sum_{i j}(\rho A)_{i j} u_{i j}^{*} \\
& -\sum_{o j}(\rho A)_{o j} u_{o j}^{*}-\sum_{j}(\rho S \Delta z) v_{j}^{*}
\end{aligned}
$$

The pressure correction equation is then simplified to Eq. 1.66 


$$
a_{p} p^{\prime}=\sum_{o v} a_{p, o v} p_{o v}^{\prime}+\sum_{i v} a_{p, i v} p_{i v}^{\prime}+\sum_{j} a_{p, j} p_{o v}^{\prime}+b_{p}
$$

With,

$$
\begin{gathered}
a_{p, o v}=(\rho A d)_{o j} \\
a_{p, i v}=(\rho A d)_{i j} \\
a_{p, j}=(\rho S \Delta z d)_{j} \\
b_{p}=\frac{\left(\rho^{0}-\rho\right) A \Delta z}{\Delta t}+\sum_{i j}\left(\rho u^{*} A\right)_{i j}-\sum_{o j}\left(\rho u^{*} A\right)_{o j}-\sum_{j}\left(\rho v^{*} S \Delta z\right)
\end{gathered}
$$

Implementation of Boundary Conditions

The user of the code will be capable of specifying fluid mass flow rates and thermo- dynamic conditions at appropriate locations within the model as a function of time. The ability to specify the mass flow rate is accomplished by using a single-junction and introducing information tables. The single-junction is similar to an individual junction within a subchannel, but the user has the ability to specify the mass flow rate through the junction as a function of time. Mass flow rate through the single- junction is obtained by linearly interpolating data from within the information table. The ability to specify thermodynamic conditions within the model is accomplished by introducing single-volumes and utilizing information tables. The single-volume is similar to an individual volume within a subchannel, but the pressure and temperature within the volume can be specified as a function of time. Pressure and temperature within the single-volume are obtained by linearly interpolating data from within the information table. 


\section{Constitutive Equations}

\section{Fluid Properties}

The properties of helium are hardcoded into the code for convenience. In addition, the user has the option of specifying a constant property fluid. Material properties for Helium are obtained using equations from Petersen [13] (also available in KTA 3102.1 [14]). These equations are provided below for completeness and are valid for pressures ranging from 1 bar to 100 bar and from temperatures ranging from $293 \mathrm{~K}$ to $1773 \mathrm{~K}$ [14].

Density of Helium

$$
\rho=48.14 \frac{p}{T}\left(1+0.4446 \frac{p}{T^{1.2}}\right)^{-1}
$$

\section{Darcy Friction Factors}

Darcy friction factors are utilized to calculate the major head loss in a turbulent ow. These friction factors are obtained using the formula from Churchill [15] provided below.

$$
\begin{gathered}
f=8\left[\left(\frac{8}{R e}\right)^{12}+\frac{1}{(A+B)^{3 / 2}}\right]^{1 / 12} \\
A=\left[2.457 \ln \frac{1}{\left(\frac{7}{R e}\right)^{0.9}+\frac{0.27 \epsilon}{D}}\right]^{16} \\
B=\left(\frac{37530}{R e}\right)^{16}
\end{gathered}
$$

\section{Gap Loss Coefficients}

The primary gas loss model utilized in AGREE is the model provided by Kaburaki and Takizuka [16]. Kaburaki and Takizuka performed an experimental study of leakage flows between HTGR fuel blocks, and developed a correlation for the gap loss coefficient which can be manipulated to 
give the form in Eq. 1.78, where Lgap is the at length of a hex block in meters, ${ }_{-}$is the size of the gap in meters, and Re is the Reynolds number of the flow through the gap.

$$
K_{\text {gap }}=36 L_{\text {gap }}^{2}\left(\frac{0.67}{\delta R e}+3.13\right)
$$

Verificiation Efforts

Several verification calculations have been performed and can be grouped into three distinct sections. The first section consists of simple calculations of steady- state incompressible flow in a pipe, where exact analytic solutions exist. The results are compared against a hand calculation to verify the correction implementation of the body and friction forces. The second section consists of solutions for abrupt area changes with and without crossflow. These calculations are compared against hand calculations to verify the correct implementation of the special models. The third section is a multichannel transient of a flow reversal and is qualitative in nature. The results of the calculation are observed to verify that the transient solver produces results in physical behavior that is qualitatively correct.

\section{1-D Pipe}

A 1-D pipe problem is fabricated with the parameters given in Table 1.1, which result in a calculated Reynolds number of $\operatorname{Re}=107$, and an associated Darcy friction factor of $\mathrm{f}=0$ 0:00815 for a smooth pipe. The resulting pressure profiles, tabulated in Table 1.2 and shown in Fig. 1.14, are in agreement with the exact solution.

Table 1.1: 1-D pipe verification problem parameters

$\begin{array}{ll}\rho & 1000 \mathrm{~kg} / \mathrm{m} 3 \\ \mathrm{u} & 2 \mathrm{~m} / \mathrm{s} \\ \mu & 1.0 \times 10^{-5} \mathrm{~kg} / \mathrm{m} / \mathrm{s} \\ \mathrm{p}_{\text {out }} & 101.3 \mathrm{kPa} \\ \text { Length } & 1 \mathrm{~m} \\ \text { Diameter } & 5 \mathrm{~cm}\end{array}$




\begin{tabular}{|c|c|c|c|c|c|c|}
\hline \multirow{2}{*}{$z, m$} & \multicolumn{2}{|c|}{ Horizontal } & \multicolumn{2}{c|}{ Vertical Down } & \multicolumn{2}{c|}{ Vertical Up } \\
\cline { 2 - 7 } & Analytic & AGREE & Analytic & AGREE & Analytic & AGREE \\
\hline 0 & 101.626 & - & 91.816 & - & 111.436 & - \\
0.05 & 101.610 & 101.610 & 92.290 & 92.290 & 110.929 & 110.929 \\
0.15 & 101.577 & 101.577 & 93.238 & 93.238 & 109.915 & 109.915 \\
0.25 & 101.544 & 101.544 & 94.187 & 94.187 & 108.902 & 108.902 \\
0.35 & 101.512 & 101.512 & 95.135 & 95.135 & 107.888 & 107.888 \\
0.45 & 101.479 & 101.479 & 96.084 & 96.084 & 106.875 & 106.875 \\
0.55 & 101.447 & 101.447 & 97.032 & 97.032 & 105.861 & 105.861 \\
0.65 & 101.414 & 101.414 & 97.981 & 97.981 & 104.848 & 104.848 \\
0.75 & 101.381 & 101.381 & 98.929 & 98.929 & 103.834 & 103.834 \\
0.85 & 101.349 & 101.349 & 99.877 & 99.877 & 102.820 & 102.820 \\
0.95 & 101.316 & 101.316 & 100.823 & 100.823 & 101.807 & 101.807 \\
B & 101.300 & 101.300 & 101.300 & 101.300 & 101.300 & 101.300 \\
\hline
\end{tabular}

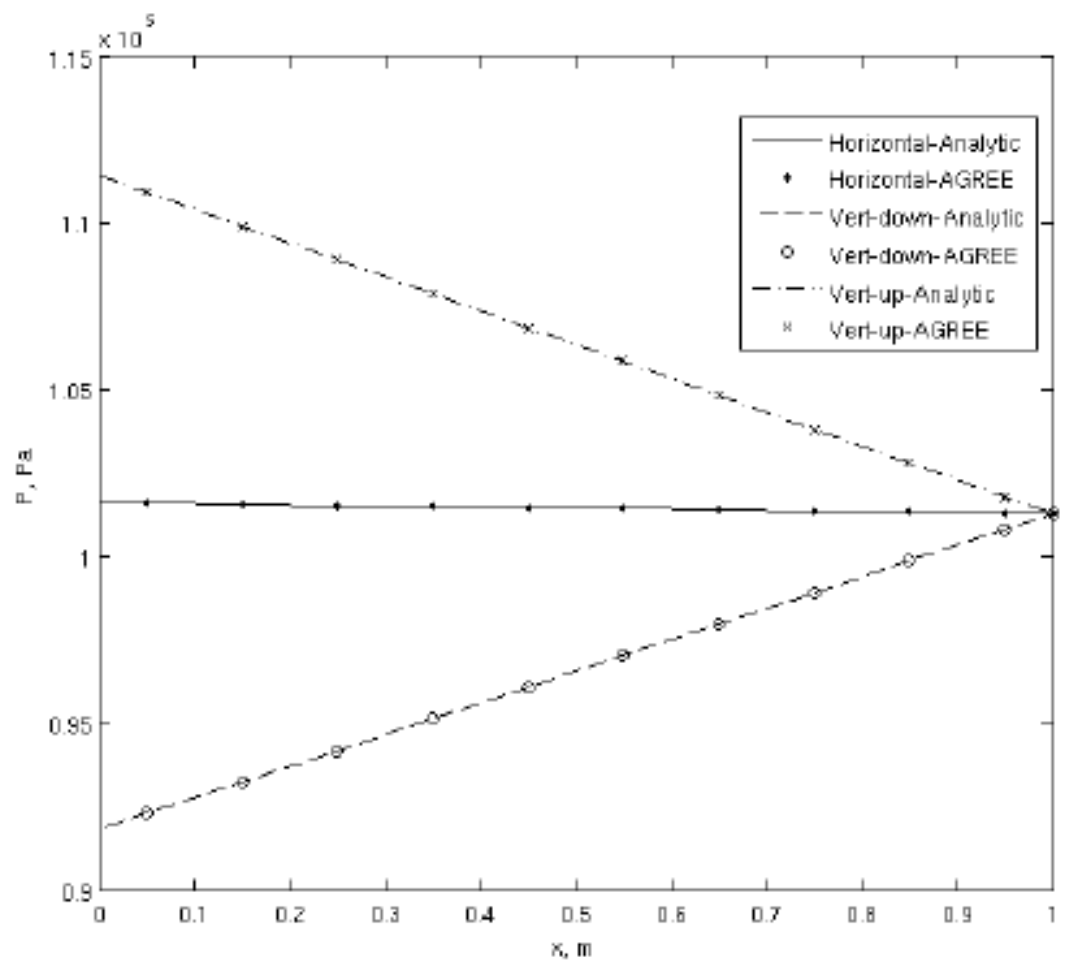

Figure 1.14: 1-D pipe verification results: pressure profile

Abrupt Area Change and Crossflow

The fabricated 1-D pipe problem is extended in the following sections to test the special models used to handle abrupt area changes and crossflow.

Abrupt Area Change 
For the abrupt area change, the calculation parameters given in Table 1.1 are largely the same, with the exception that the pipe is lengthened and an expansion that doubles the pipe diameter is inserted into the middle of the original pipe. The results are shown for forward and reverse flows in Fig. 1.15 and Fig. 1.16 respectively and are in agreement with the exact solution. It is noted that two cases were run in AGREE. In AGREE-1, the abrupt area change is across two different sized channels. In AGREE-2, the abrupt area change occurs coincident with a zero volume node.

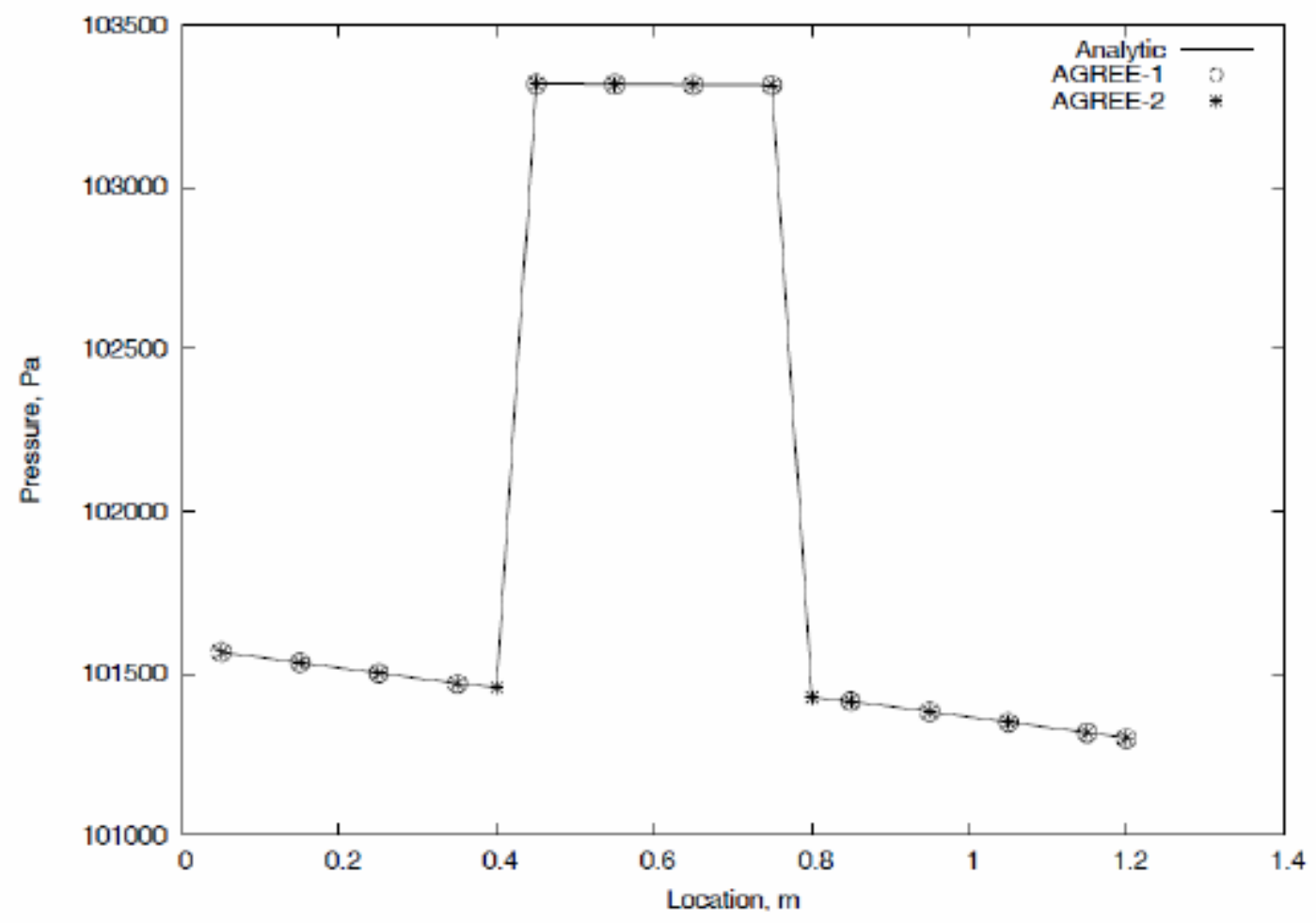

Figure 1.15: Verification calculation for abrupt area change model(forward) 


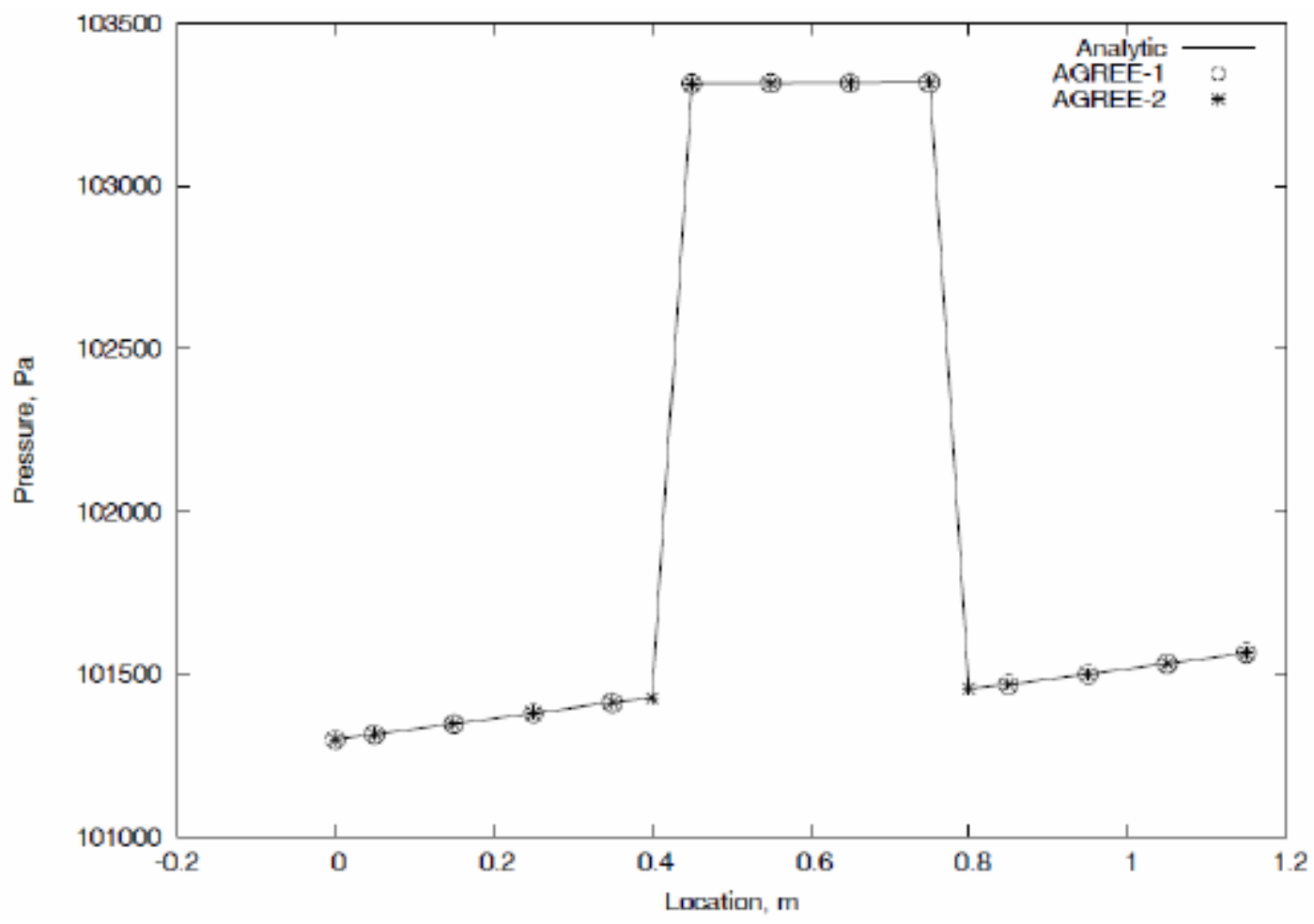

Figure 1.16: Verification calculation for abrupt area change model(reverse)

\section{Crossflow}

For the cases with cross ow, two identical channels are merged together. Three cases are tested: no area change; a contraction coincident with crossflow; and an expansion coincident with crossflow. The results are shown for forward and reverse flows in Fig. 1.17 and Fig. 1.18 respectively and are in agreement with the exact solution. 


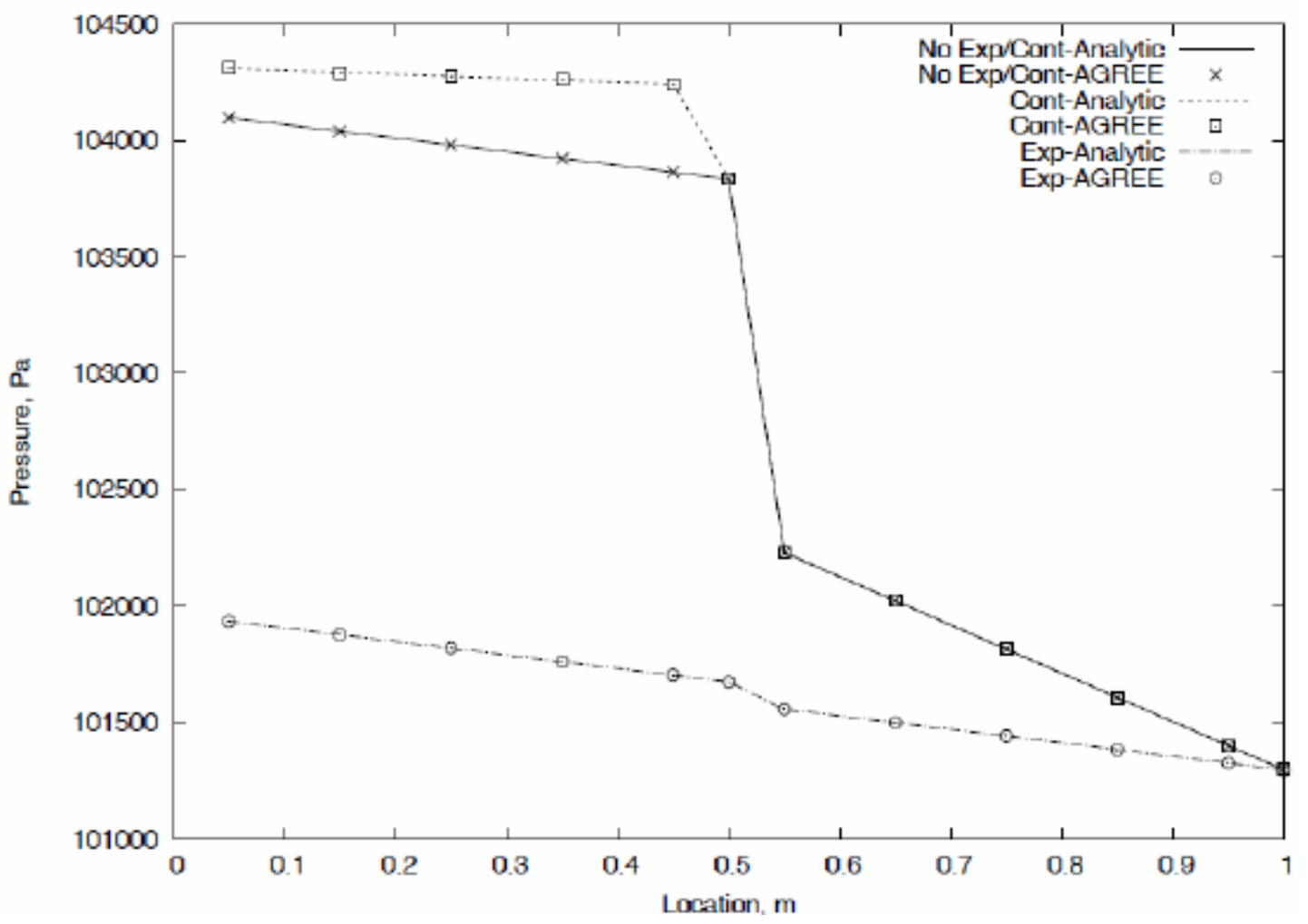

Figure 1.17: Verification of crossflow (forward)

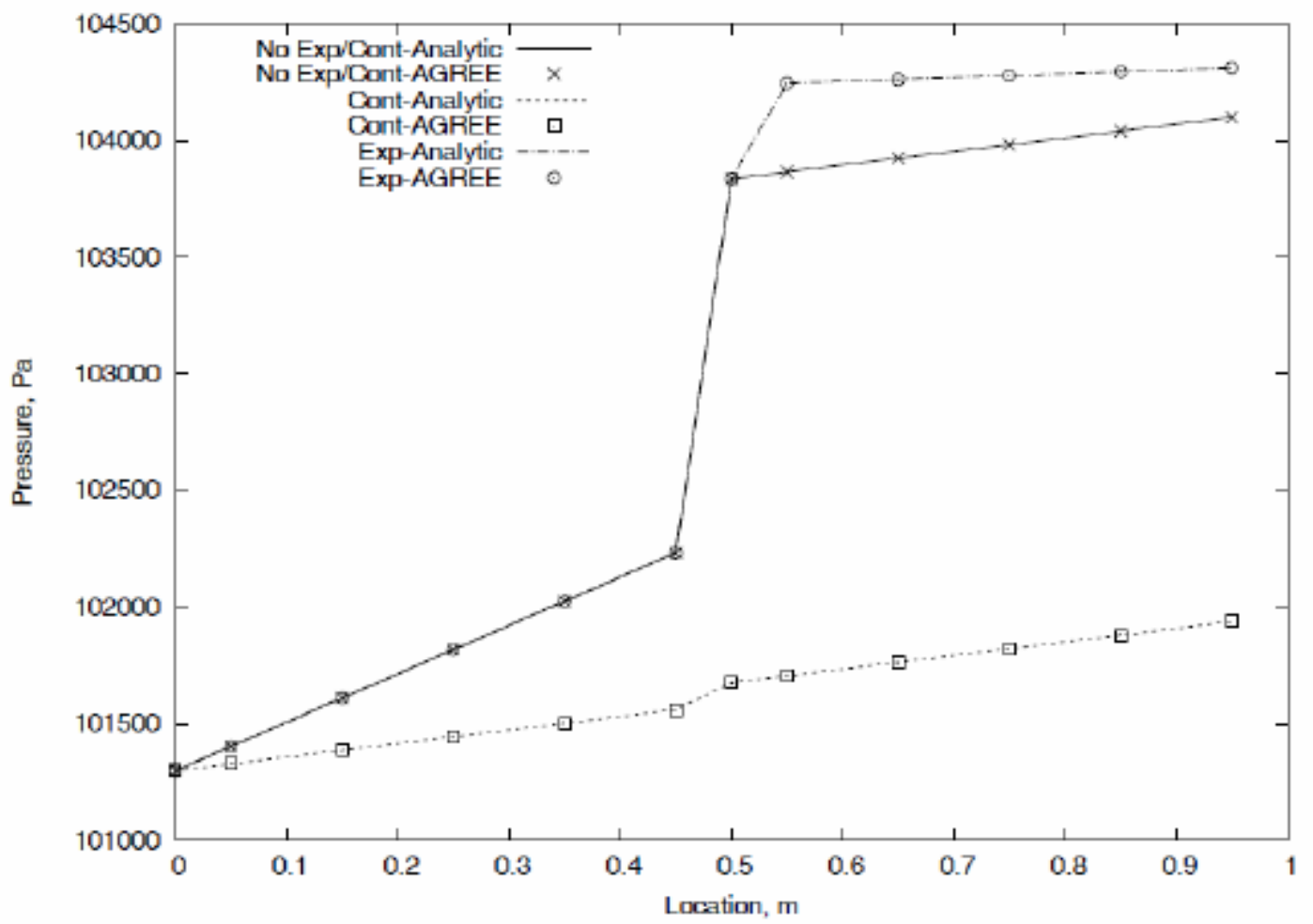

Figure 1.18: Verification of crossflow (reverse) 


\section{Flow Reversal Transient}

In order to verify the proper behavior of the transient solver, a multichannel transient is fabricated where the steady-state solver is used in the forward direction and then the pressure boundary conditions are ipped and a transient is run in the reverse direction. The problem consists of six identical channels and is shown in Fig. 1.19 with the pressures in the inlet and outlet volumes, specified as a function of time, are shown in Fig. 1.20. The resulting pressure profiles, shown in Fig. 1.21, demonstrate that the achieved steady-state profile in the reverse direction is in agreement with the forward steady-state profile and the pressure wave propagation demonstrates physical behavior.

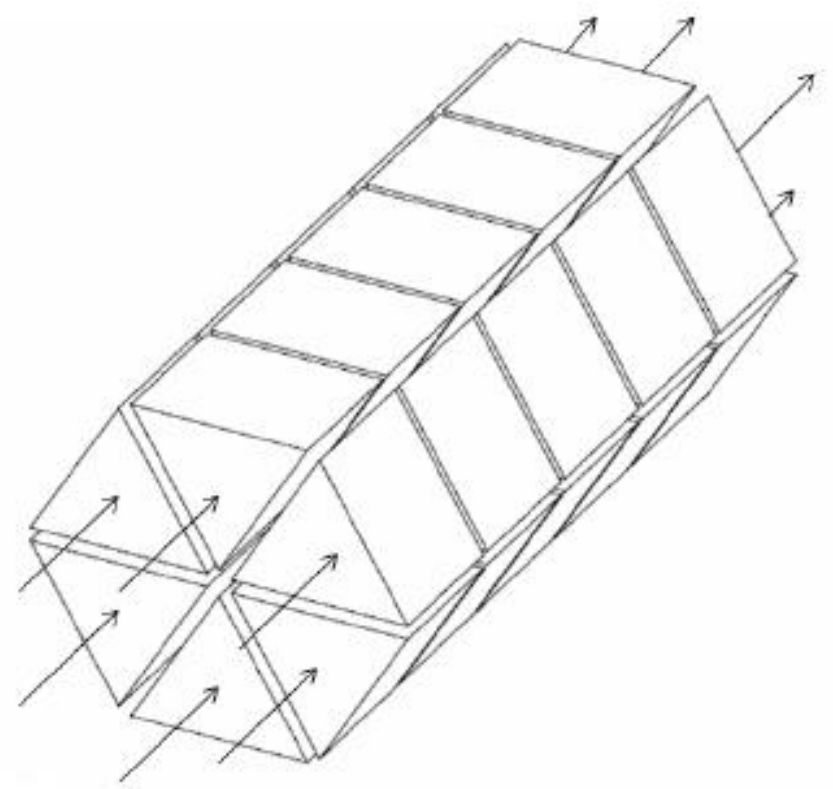

Figure 1.19: Flow reversal transient verification problem 


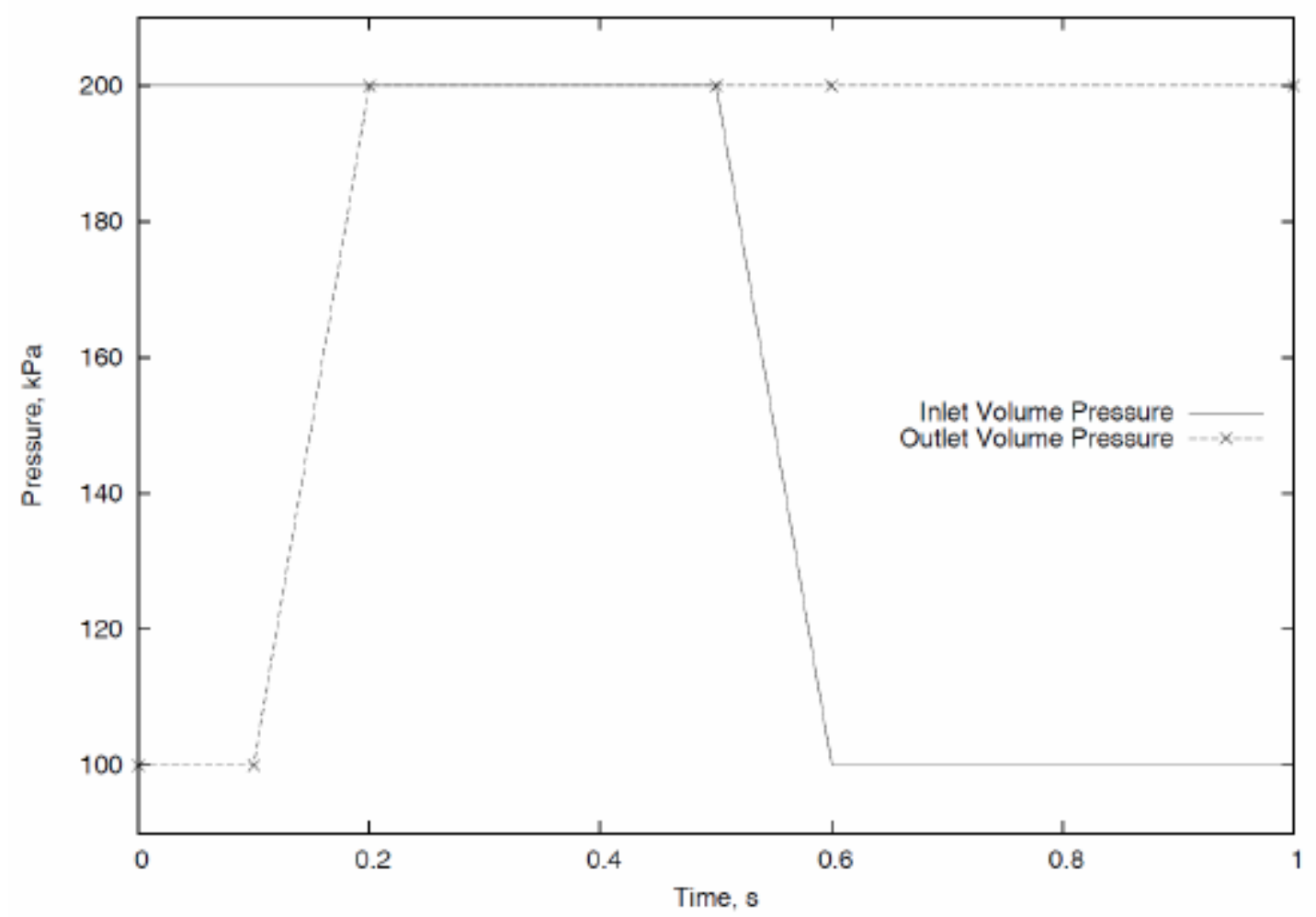

Figure 1.20: Inlet and outlet pressures for flow reversal transient

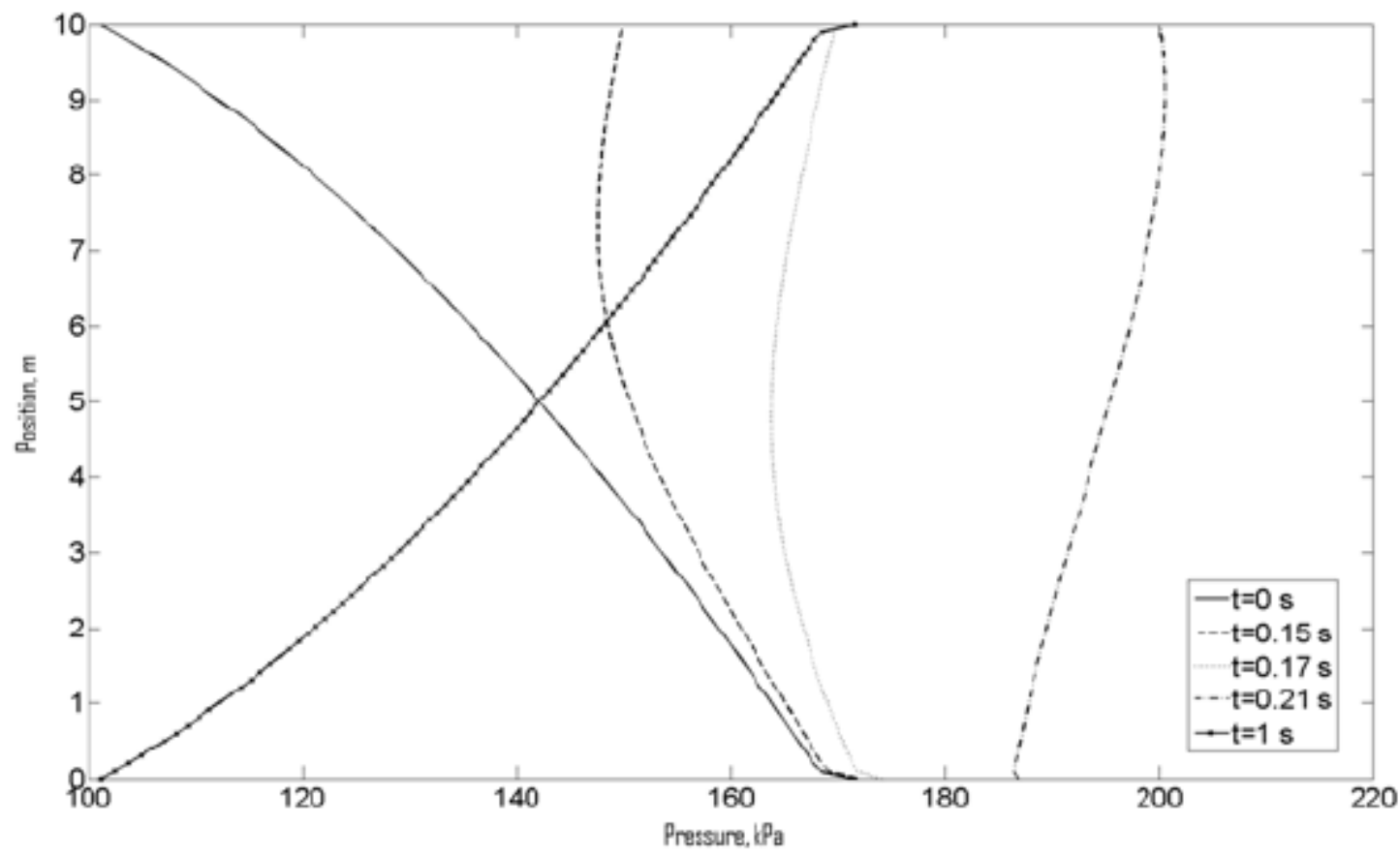

Figure 1.21: Results of reversal transient calculation

\section{Validation Efforts}


The validation calculations are grouped into two sections. In the first section, a calculation of the bypass flow tests performed by Kaburaki and Takizuka [16] is performed and the resulting pressure profile is compared against the data published in the literature. In the second section, a much more comprehensive set of validation calculations are performed and compared against experimental data and obtained from Seoul National University (SNU). The SNU results are also compared against other codes/methods for contrast.

\section{Kaburaki and Takizuka}

The calculation is performed for a bypass flow test performed by Kaburaki and Takizuka [16] and represents four full size HTTR fuel elements surrounded by a metal shroud that simulates a 1-mm bypass flow gap with a crossflow gap imposed between the 2 nd and 3 rd fuel element, see Fig. 1.22. To perform the calculation an initial analytic study was performed to obtain simulation parameters from the data. Kaburaki and Takizuka measured the mass flow rate to be $182 \mathrm{~g} / \mathrm{s}$. Using the measured mass flow rate and the pressure drop at the interface between the 3rd and 4th blocks, the form loss coefficient for the combined expansion/contraction is calculated to be approximately 0.3 . The pressure drop at the entrance due to fluid acceleration and the entrance loss coefficient is obtained utilizing the value of $9 \%$ for the bypass ow, which had been determined by Kaburaki and Takizuka using a flow network calculation. An entrance form loss coefficient of 0.4 is obtained from the calculation. In a manner similar to the original work of Kaburaki and Takizuka, the hydraulic diameter is adjusted to consider the effect of the insertion of the pressure tube. In combination with a friction factor obtained for a smooth pipe, a hydraulic 


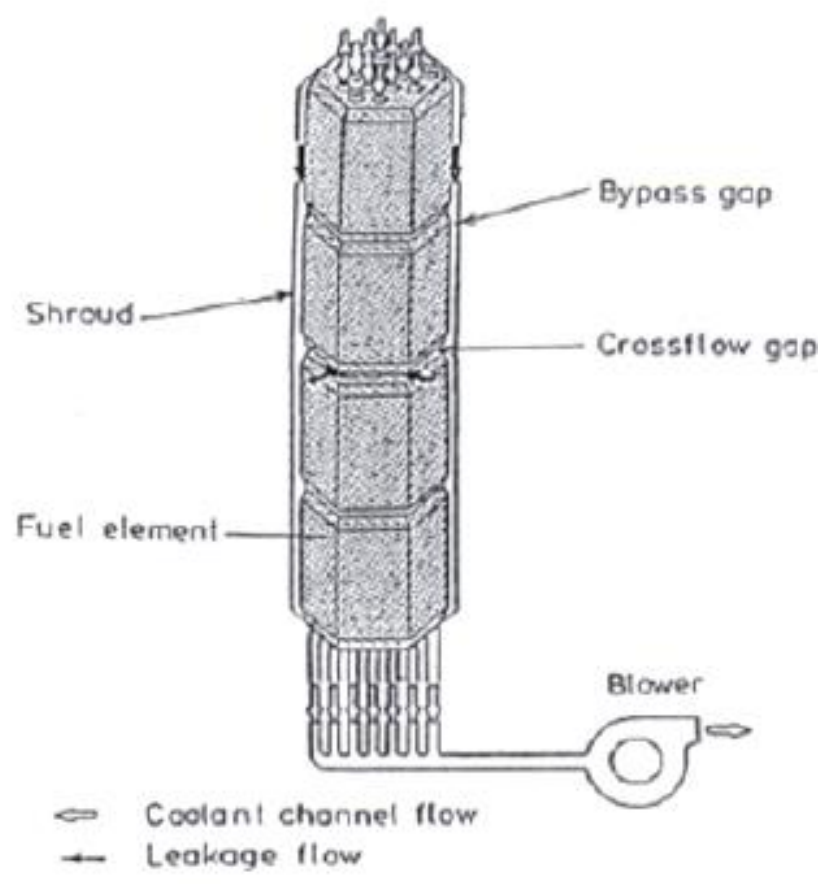

Figure 1.22: Bypass flow experiment of Kaburaki and Takisuka [16]

diameter of $1.6 \mathrm{~cm}$ is found to produce a satisfactory slope for the pressure profile. For the flow between the block and shroud, the pressure drop is obtained using the analytic solution for laminar flow between parallel plates given by Eq. 1.79, where $\mathrm{u}$ is the mean velocity of the fluid, 1 is the friction length, and $t$ is the gap thickness. The Reynolds number in bypass flow channel has been calculated to be 1730 .

$$
\Delta p=\frac{12 \mu u}{t^{2}} l
$$

The results from the analysis with AGREE are shown in Fig. 1.23. A mass flow rate of $182 \mathrm{~g} / \mathrm{s}$ with a bypass flow of $11.3 \%$ is calculated. The bypass flow over- predicts the $9 \%$ value stated by Kaburaki and Takizuka, which was obtained by a flow network calculation, by $2.3 \%$. The data from Kaburaki and Takizuka does not include any information regarding uncertainty in the measurements; therefore, it is difficult to make statements regarding the accuracy of the calculation in regard the physical data. However, it can be observed that the pressure profile in the main coolant and bypass flow channels produced by the AGREE calculation demonstrate the same physical trends as observed in the experimental data. In particular, the large pressure drop attributed to the flow acceleration at the entrance to each flow channel is observed and appears to be correct in magnitude. The major friction losses within the flow channels appear to match the profiles well, where the Churchill correlation is used to obtain the friction factor within the 
turbulent regime found in the main coolant channel, and laminar flow is observed in the bypass channel. Stagnation in the bypass channel and the resulting flow acceleration that results from the merging crossflow into the main coolant channel is observed and appears to be correct in magnitude.

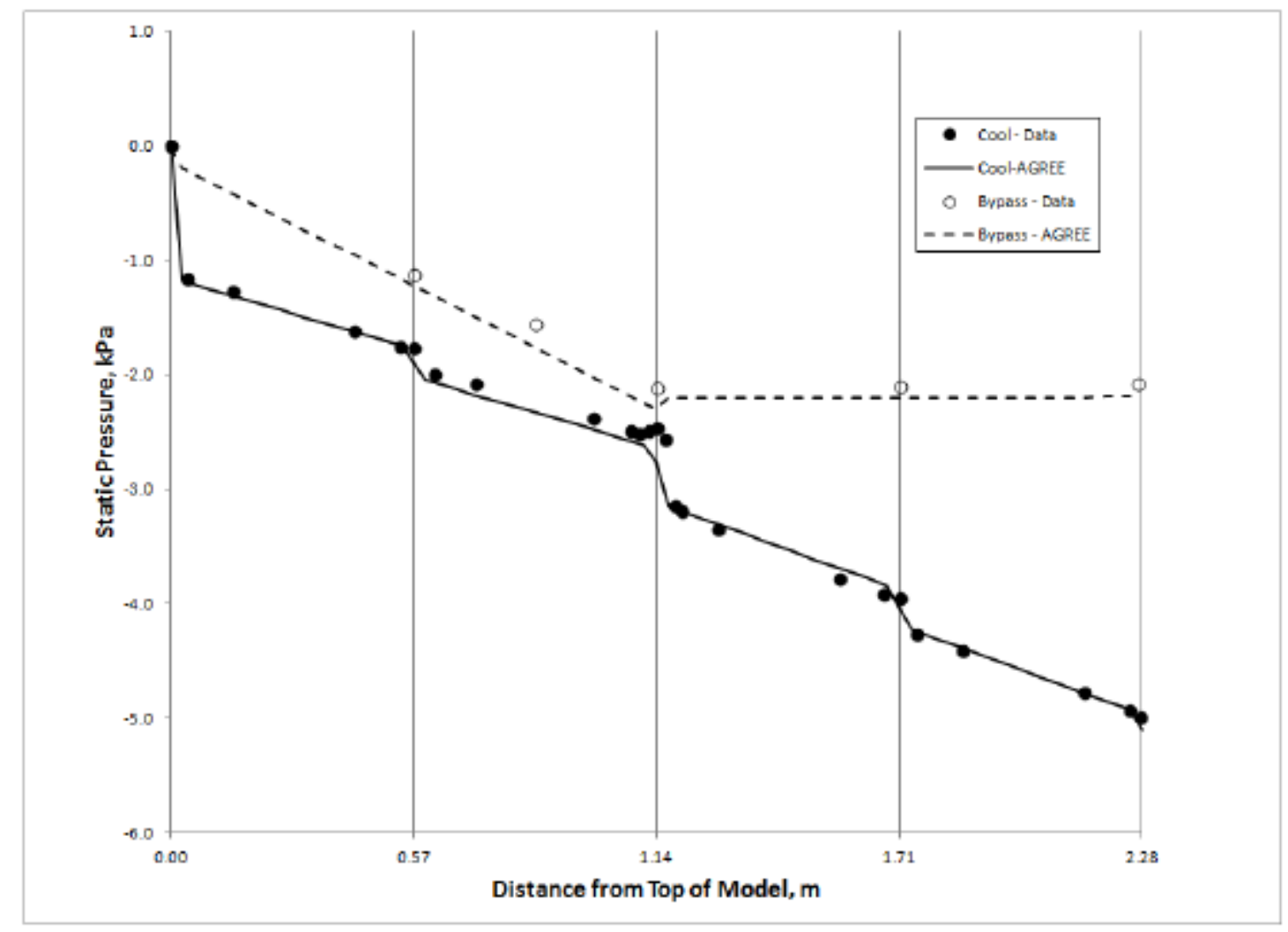

Figure 1.23: AGREE calculation of bypass flow experiment of Kaburaki and Takisuka

\section{SNU Bypass Flow Tests}

In order to understand the bypass flow behavior and to generate an experimental database for the validation of computer codes, a series of experiments were performed at Seoul National University (SNU) using atmospheric air [17, 18, 19, 20, 21, 22 23]. The numerical calculations using the AGREE code is compared against other codes and with the measured data in this section. The bypass flow experiments at SNU are classified into three phases and discussed below.

It will be mentioned here that at the time these calculations were originally per- formed, not all of the features currently available in AGREE were present. The features that impact these calculations are the special models (see Section 1.3.2) that were implemented as a consequence of the phase III results, and the card based input structure that removes the input limitations discussed in some of the tests. The results presented for phase I reect the current state of 
AGREE, but the results for phase II and phase III represent a version of the code that does not include the aforementioned code features.

SNU Bypass Flow Test - Phase I

Instead of entire prismatic core, the bypass flow phase I experiment at SNU fo- cused on a triangular unit cell of the core. As shown in Fig. 1.24, the unit cell is a regular triangle section that is formed by connecting the center points of three hexag- onal blocks in the core. The main phenomenon captured in the phase I experiment was the flow split between the coolant channel and the bypass gap. Three sizes of bypass gaps (i.e., 1, 3, $5 \mathrm{~mm}$ ) were used to examine the effect. Cross flow was not considered in the phase I experiment. The facility used in the phase I experiment is shown in Fig. 1.25. It is an open-loop facility. The working fluid was atmospheric air at room temperature. The total length and width of the experimental facility were 7:75 $\mathrm{m}$ and 1:40 m, respectively. The length of the test block was $79 \mathrm{~cm}$, and the cross section of the test section was an equilateral triangle of $43 \mathrm{~cm} .1 / 3$ section of the triangle has 23 coolant holes in the case of fuel block. The test section and test blocks were made of acryl. The air flow rates were measured at the inlet of the blower and each flow measuring tube, which was installed at the downstream of the coolant channel flow of the individual test block. The bypass flow was determined by the subtraction of the coolant channel flow from the inlet ow. More detailed description of the phase I experiment is available in Refs. [17, 18, 19]. Among various block combinations considered in the experiment, F3 case having three fuel columns with different gap sizes and mass flow rates was selected for the present study since smaller uncertainties were expected in $\mathrm{F} 3$ case.

Table 1.3: Comparison of calculated and measured bypass flow fractions for SNU phase I experiment

\begin{tabular}{|c|c|c|c|c|c|c|c|}
\hline \multirow[b]{2}{*}{$\begin{array}{l}\text { Test } \\
\text { Case }\end{array}$} & \multirow[b]{2}{*}{$\begin{array}{c}\text { Gap } \\
\text { size } \\
(\mathrm{mm})\end{array}$} & \multirow[b]{2}{*}{$\begin{array}{c}\text { Mass } \\
\text { Flow-rate } \\
(\mathrm{kg} / \mathrm{s})\end{array}$} & \multicolumn{5}{|c|}{ Bypass Flow (\%) } \\
\hline & & & $\begin{array}{c}\text { Experiment } \\
{[19]}\end{array}$ & AGREE & $\begin{array}{c}\text { GAMMA+ } \\
{[24]}\end{array}$ & $\begin{array}{c}\text { CFX } \\
{[19]}\end{array}$ & $\begin{array}{c}\text { STAR-CD } \\
{[25]}\end{array}$ \\
\hline G1F3W1 & 1 & 0.1801 & 1.19 & 0.94 & 0.33 & 0.41 & 0.32 \\
\hline G3F3W1 & 3 & 0.1863 & 4.75 & 5.67 & 5.62 & 4.29 & - \\
\hline G5F3W1 & 5 & 0.1911 & 10.99 & 11.03 & 11.03 & 10.31 & 10.57 \\
\hline G1F3W2 & 1 & 0.3736 & 1.39 & 1.11 & 0.63 & 0.64 & 0.51 \\
\hline G3F3W2 & 3 & 0.3843 & 4.89 & 5.88 & 5.50 & 4.99 & - \\
\hline G5F3W2 & 5 & 0.3878 & 10.46 & 11.18 & 11.38 & 10.35 & 9.46 \\
\hline G1F3W3 & 1 & 0.5560 & 1.22 & 1.16 & 0.92 & 0.77 & 0.61 \\
\hline G3F3W3 & 3 & 0.5700 & 4.46 & 6.00 & 5.69 & 5.26 & - \\
\hline G5F3W3 & 5 & 0.5802 & 10.70 & 11.29 & 11.60 & 10.31 & 10.08 \\
\hline
\end{tabular}

Existing CFD calculations by SNU [19] and Argonne National Laboratory (ANL) [25] and GAMMA+ calculations [24] are available and are provided to show the relative accuracy of AGREE. CFX and STAR-CD are commercial computational fluid dy- 
namics codes. GAMMA+ is a VHTR system code that models the reactor on a scale similar to AGREE [26, 27, 28]. The tabulated results, shown in Table 1.3, show that the results from AGREE compare well with experiment. The results for all codes do not demonstrate any clear bias when compared against experimental data, i.e. bypass owrates are over-predicted for some cases and under-predicted for others. It is noted that the tabulated results make no statement in regards to uncertainty in experimental data or computation.

SNU Bypass Flow Test - Phase II

A schemcatic of the bypass flow phase II experiemnt is shown in Fig. 1.26. The layout of the phase II facility is similar to that of the phase I except the test section. The test section of the phase I experiment consisted of a single layer without any gaps. Therefore, multi-block effects such as cross-ow phenomenon or lateral flow around block periphery, could not be examined in the phase I experiment. In order to investigate such multi-block effects, multi-column and multilayer test sections were fabricated in the phase II experiment. In other words, cross flow behavior between the bypass gap and the coolant channel as well as lateral flow behavior around the bypass gaps were the focus in the phase II experiment. The test section for the phase II experiment is shown in Fig. 1.27. The phase II experiments utilized three types of test sections. The cross-sections of these test sections are shown in Fig. 1.28. Additionally, an acrylic test block and the coolant hole geometry are shown in Fig. 1.29 and Fig. 1.30 respectively.

Each test section represents different combinations of two types of test blocks (e.g., fuel and reector type blocks). The test section of the experiment consists of three layers and 11 columns of acrylic test blocks. Each layer includes three hexagonal blocks in the middle row, on each side of which there are two pentagonal and rectangular blocks. The dimension of the test block was scaled down to one-third of the real block. The at-to-at width and height of the hexagonal test block were $120 \mathrm{~mm}$ and $264 \mathrm{~mm}$, respectively. The bypass gap size was fixed to be $2 \mathrm{~mm}$. Three cross flow gap sizes of $0,1 \mathrm{~mm}$, and $2 \mathrm{~mm}$ were tested. The inlet mass flow rate of the test section and outlet mass flow rate of each block column were measured to evaluate the bypass flow fraction. In contrast to the phase I experiment, the local pressure distribution was measured in the phase II along the bypass gap channel. As shown in Fig. 1.31, a total of 14 pressure taps were installed on the both side walls of the test section along the central bypass gap. More detailed descriptions of the phase II experiment are available in Refs. [18, 20].

Among various cases of the phase II experiment, six cases were considered for AGREE validation. The considered cases are summarized in Table 1.4. The geometry of the coolant holes and bypass gaps was modeled in detail. Five coolant holes in the pentagonal test block were modeled by four coolant holes to match the cross flow junctions. Fig. 1.32 shows the axial nodalization for the AGREE calculations. Due to the input limitations of AGREE at the time of the calculations, the region downstream of the coolant channels (which consists of the mixing chamber and the measuring tube) cannot be modeled. Therefore, the form loss factor was applied 
to consider the flow resistance after exiting the coolant channels of the test section. The form loss factor was adjusted by the case without the cross flow gap.

Table 1.4: Phase II experiments considered for AGREE validation

\begin{tabular}{|c|c|c|c|c|}
\hline Test case & $\begin{array}{c}\text { Test section } \\
\text { (see Fig. 1.28) }\end{array}$ & $\begin{array}{c}\text { Mass flow rate } \\
(\mathrm{kg} / \mathrm{s})\end{array}$ & $\begin{array}{c}\text { Gap Size } \\
(\mathrm{mm})\end{array}$ & Reference \\
\hline F3CG0L & F3 & 0.4226 & 0 & {$[18,22,29,30]$} \\
F3CG0H & F3 & 0.5729 & 0 & {$[18,20,22]$} \\
F3CG2L & F3 & 0.4179 & 2 & {$[18,22,29,30]$} \\
F3CG2H & F3 & 0.5628 & 2 & {$[18,20,22]$} \\
\hline F1CG0 & F1 & 0.1783 & 0 & {$[18,22]$} \\
F1CG2 & F1 & 0.1775 & 2 & {$[18,22,29]$} \\
\hline
\end{tabular}

Fig. 1.33 through Fig. 1.38 show the results of the AGREE calculations for the phase II experiment. The results are compared with the measured values. The existing CFX and GAMMA+ results are provided for the cases that are available in the literature. Fig. 1.33 shows the AGREE result for the F3CG0L case. In order to obtain the best fit of the experimental data, the form loss factor at the outlet of the coolant channel was adjusted to be 4.5 , which is utilized for all cases utilizing the F3 test section. With the same form loss factor, a good agreement is found for the $\mathrm{F} 3 \mathrm{CGOH}$ case, results shown in Fig. 1.34, which has the flow rare increased $36 \%$.

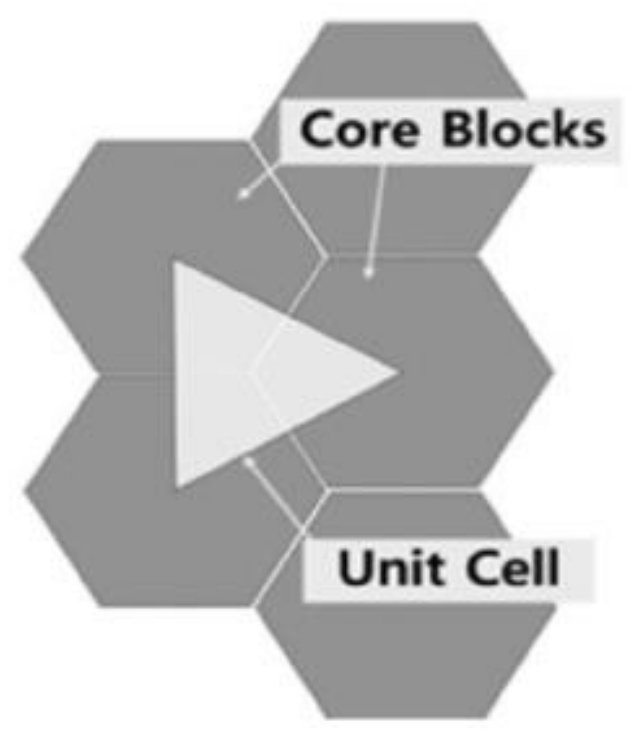



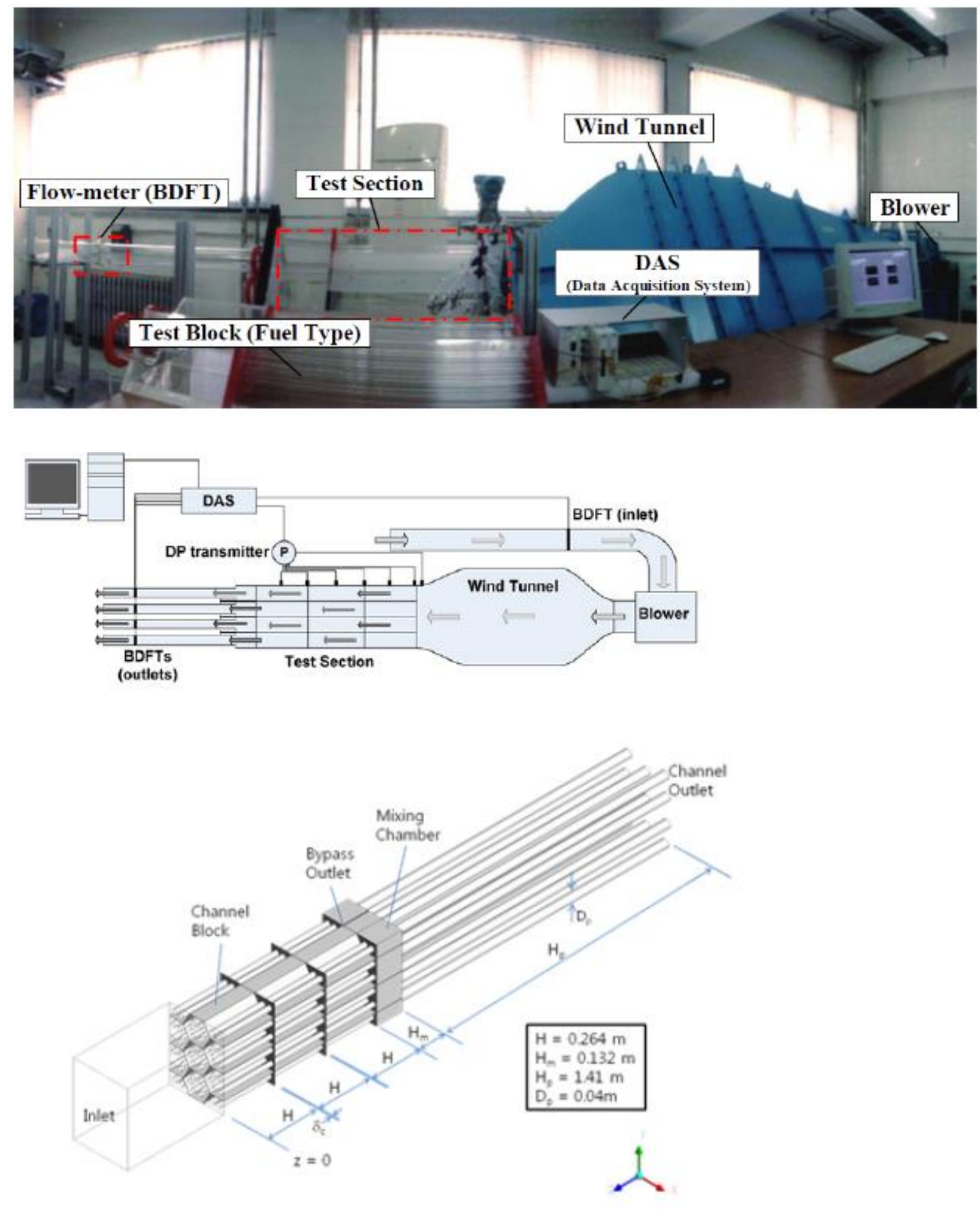
F3 Case

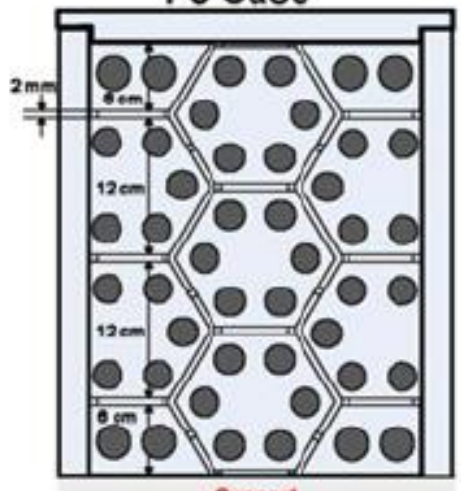

Support
F2 Case

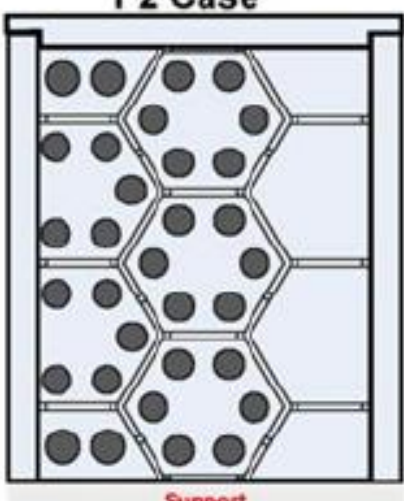

Support
F1 Case

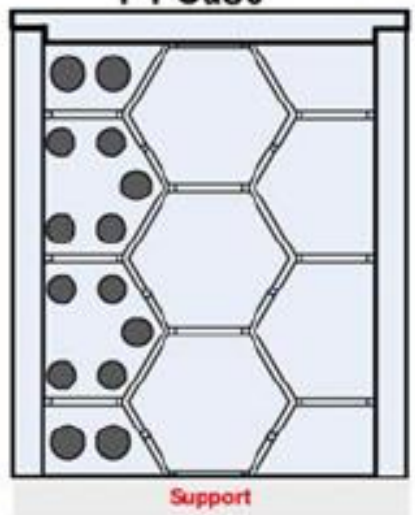

Test Section Shroud

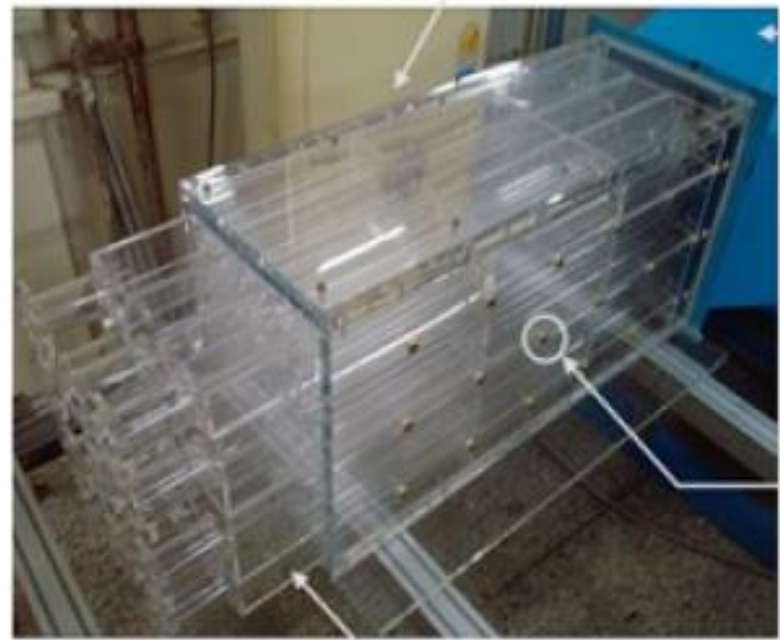

Wind Tunnel

Static Pressure

measurement Tab

Test Block(Fuel type)
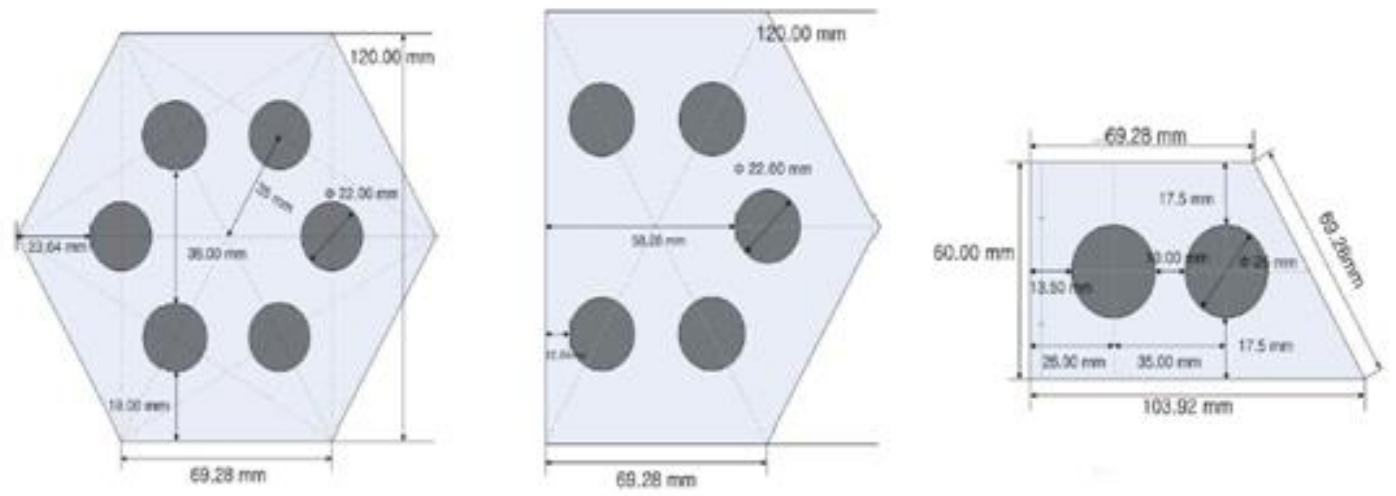

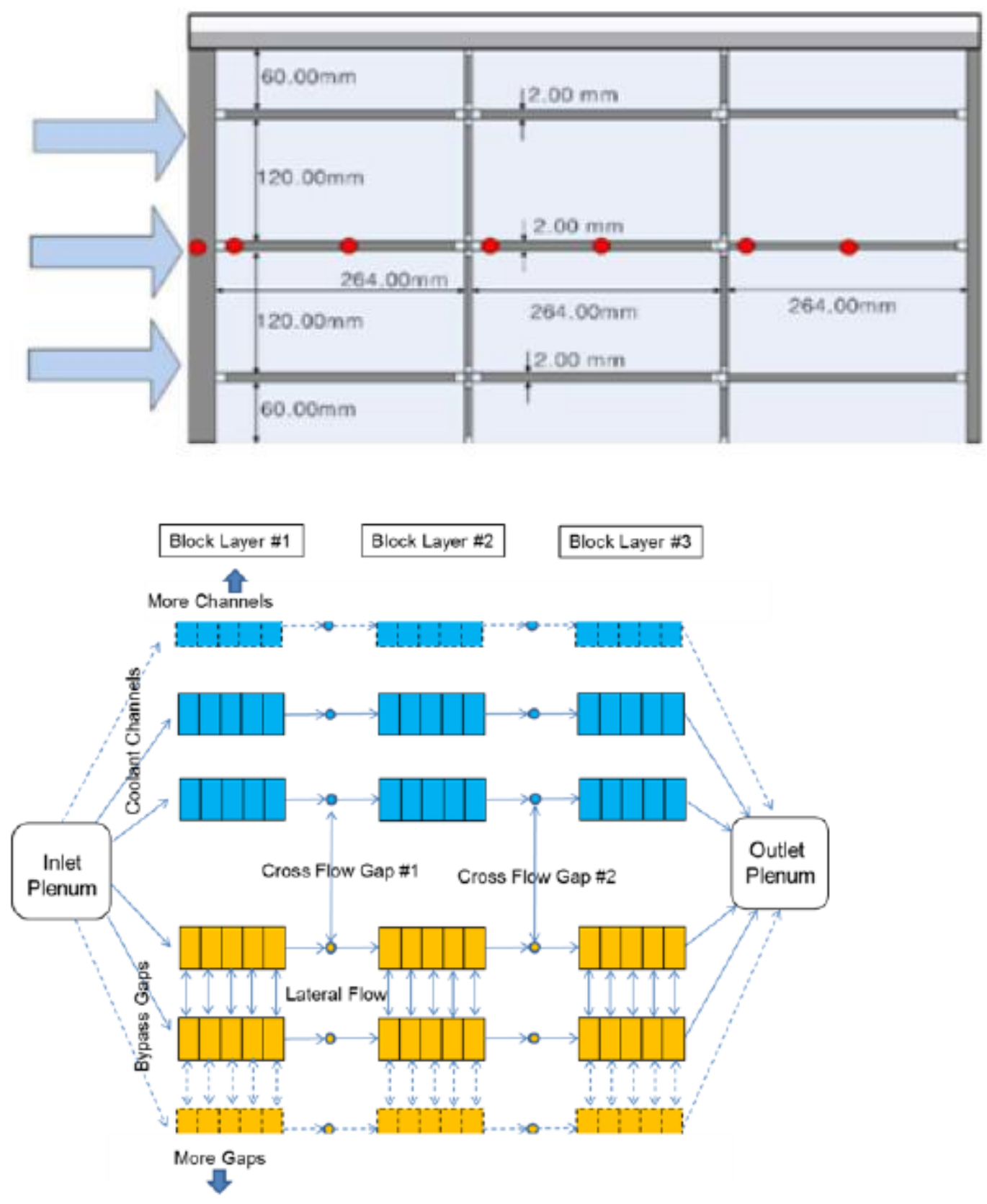

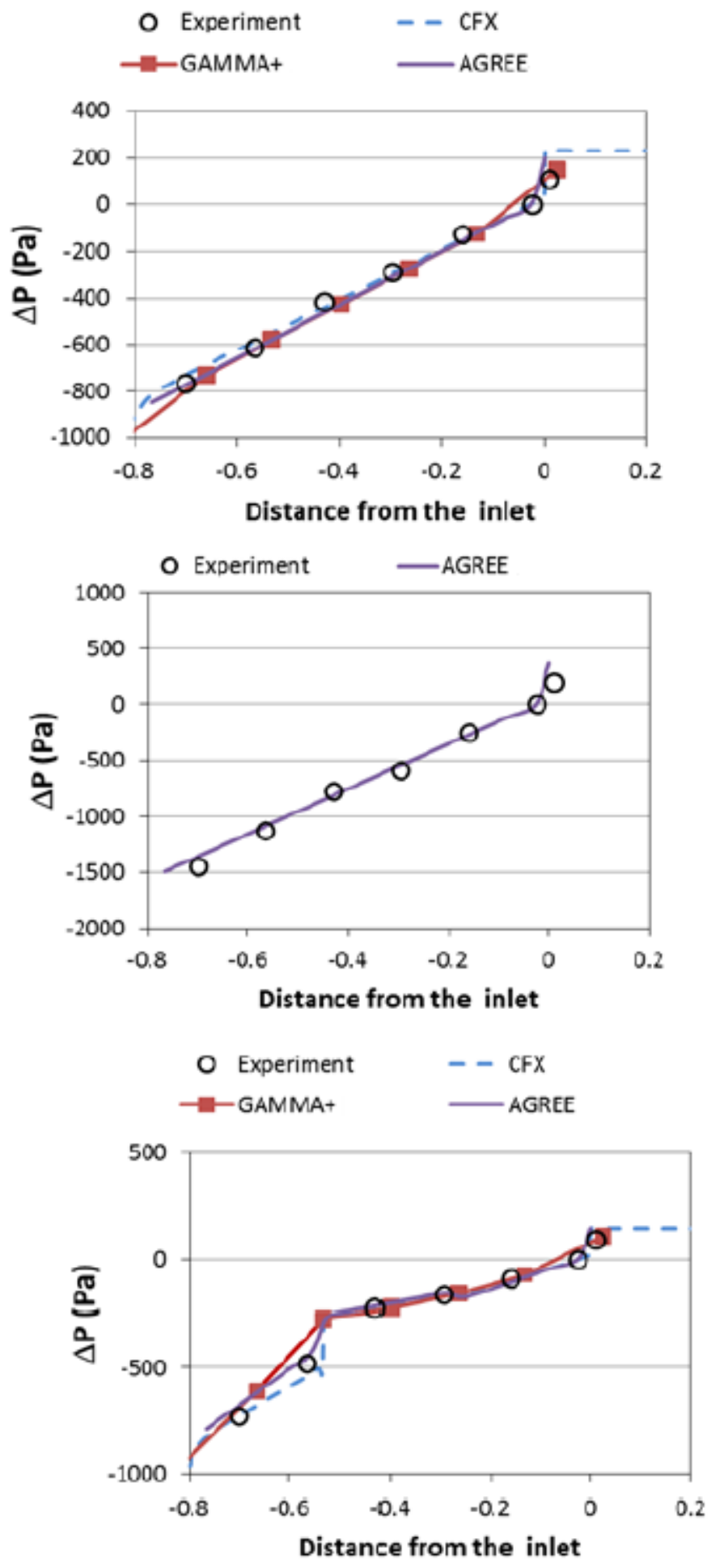


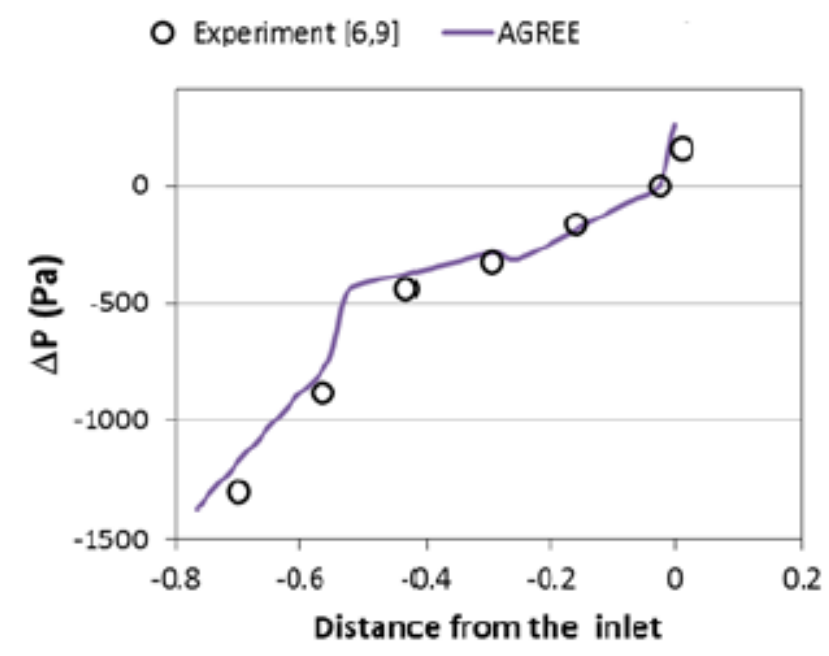

\subsection{HTR Heat Transfer Model in AGREE}

This subsection will describe the Heat Transfer model developed and implemented in AGREE. The control volume used to apply the triangular finite volume method is shown in Fig. 5.

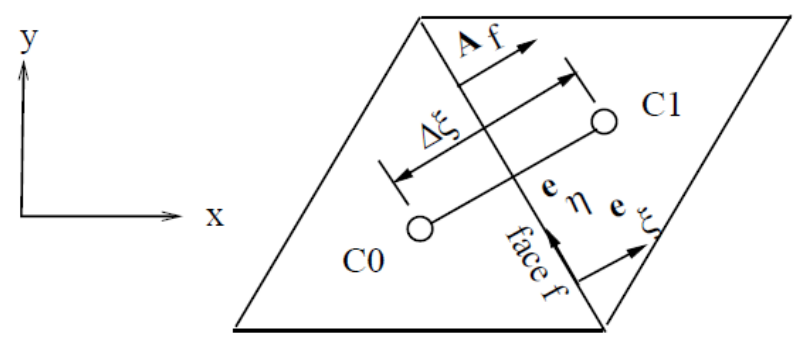

Fig. 5: Control volume for triangular finite volume

By considering a simple steady state conduction equation and applying the method as follows, the resulting discrete equation for the node of the control volume can be obtained.

$$
\begin{gathered}
\nabla . J=S \\
J=-k \nabla T
\end{gathered}
$$




$$
\begin{gathered}
\int_{V} \nabla \cdot J d V=\int_{V} S d V \\
\int_{A} J \cdot d A=\int_{V} S d V \\
\sum_{f} J_{f} \cdot A_{f}=S \Delta V \\
J_{f} \cdot A_{f}=-k_{f} A_{f}\left(\frac{\partial T}{\partial \xi}\right)_{f}
\end{gathered}
$$

In the case of HTGRs, the interface between the neighboring triangles is not always continuous solid. The gaps between the hexagonal blocks create extra heat resistance. So the form of the discrete equation can be represented as follows.

$$
\begin{gathered}
k_{f} A_{f}\left(\frac{T_{o}-T_{s o}}{\frac{\partial \xi}{2}}\right)_{f}=\frac{T_{o}-T_{s o}}{R_{o}} \\
k_{f} A_{f}\left(\frac{T_{1}-T_{s 1}}{\frac{\partial \xi}{2}}\right)_{f}=\frac{T_{1}-T_{s 1}}{R_{1}} \\
k_{f} A_{f}\left(\frac{\partial T}{\partial \xi}\right)_{f}=\frac{T_{o}-T_{1}}{R_{o}+R_{1}+R_{e x}}
\end{gathered}
$$

\section{Verification of Heat Transfer with COMSOL Comparison}

As shown in Figure 8, an MHTGR 2-D, one twelfth core model was built with COMSOL. The model contains 5.5 fuel assemblies where all the fuel and coolant channels are explicitly defined. A surface-to-surface radiation model is applied to the coolant holes, reserve shutdown channels, control rod holes and the gap between the core barrel and reactor pressure vessel. In order to take 
into account the heat transfer in the gaps between the blocks, between the permanent side reflector and core barrel and between the fuel compact and fuel channel wall, this thermally resistance layers were used.

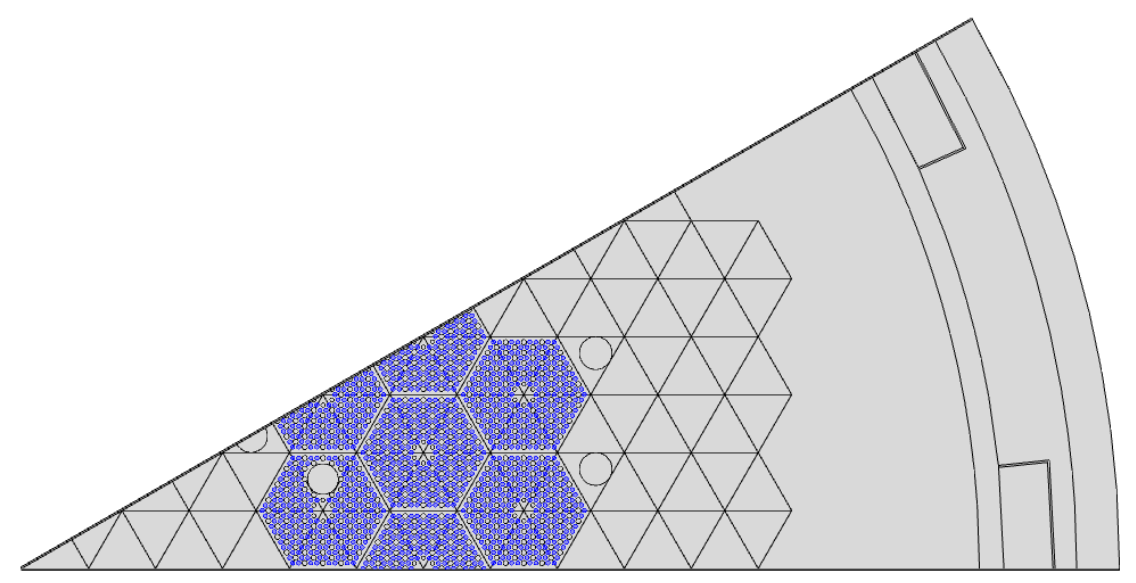

Fig. 9: COMSOL model of MHTGR.

The temperature profile from the COMSOL solution is depicted in Fig. 10. The comparison between COMSOL and AGREE is done on the average hexagonal block and average fuel block triangle temperatures. 


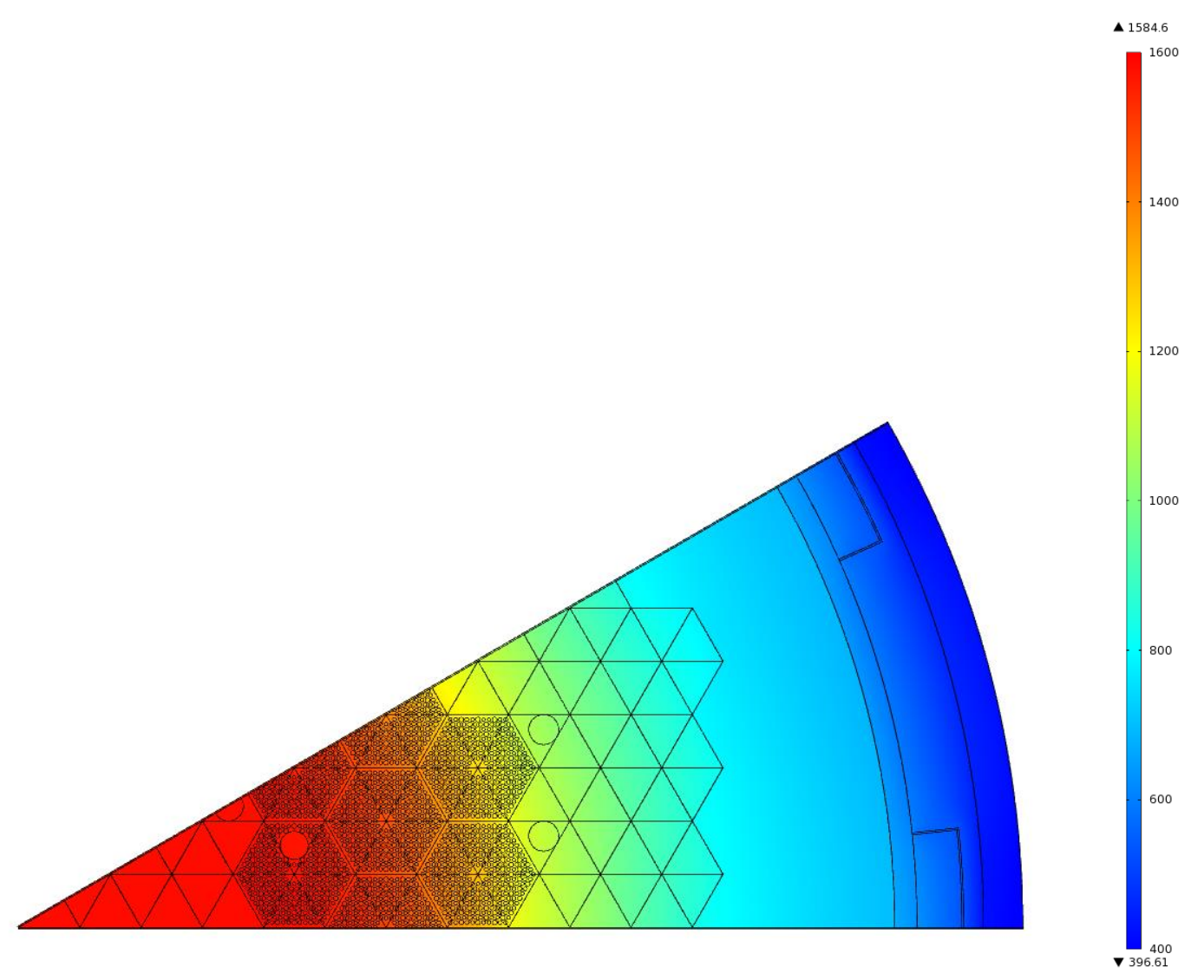

Fig. 10: COMSOL Solution.

The numbering scheme for the comparison is presented in Figure 11. The results of the AGREE/COMSOL comparison is shown in Figure 12-13.

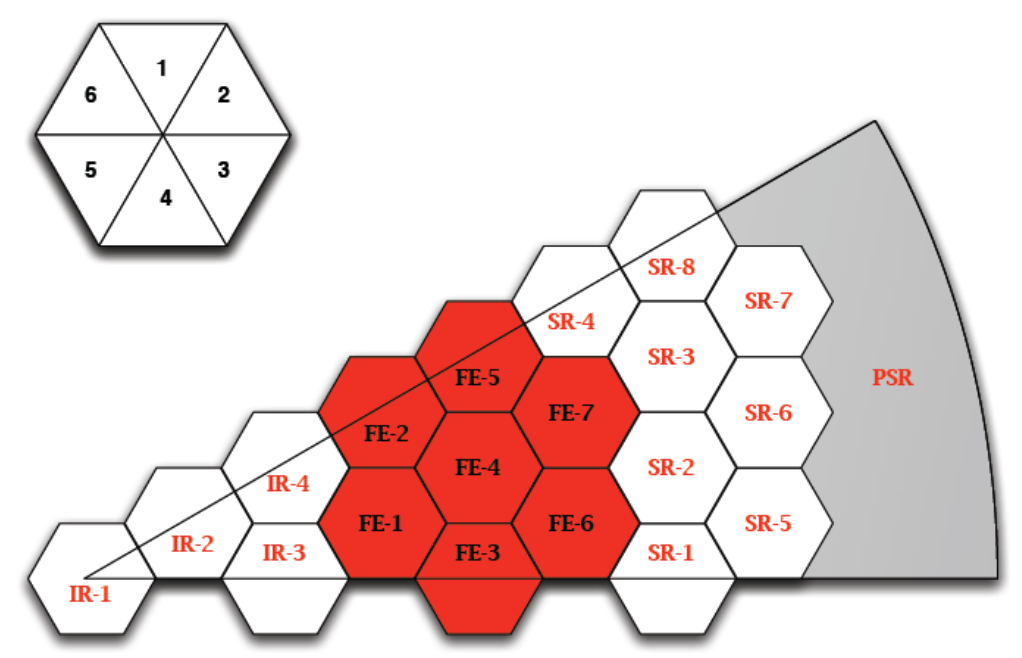

Fig. 11:.Numbering Scheme for comparisons 


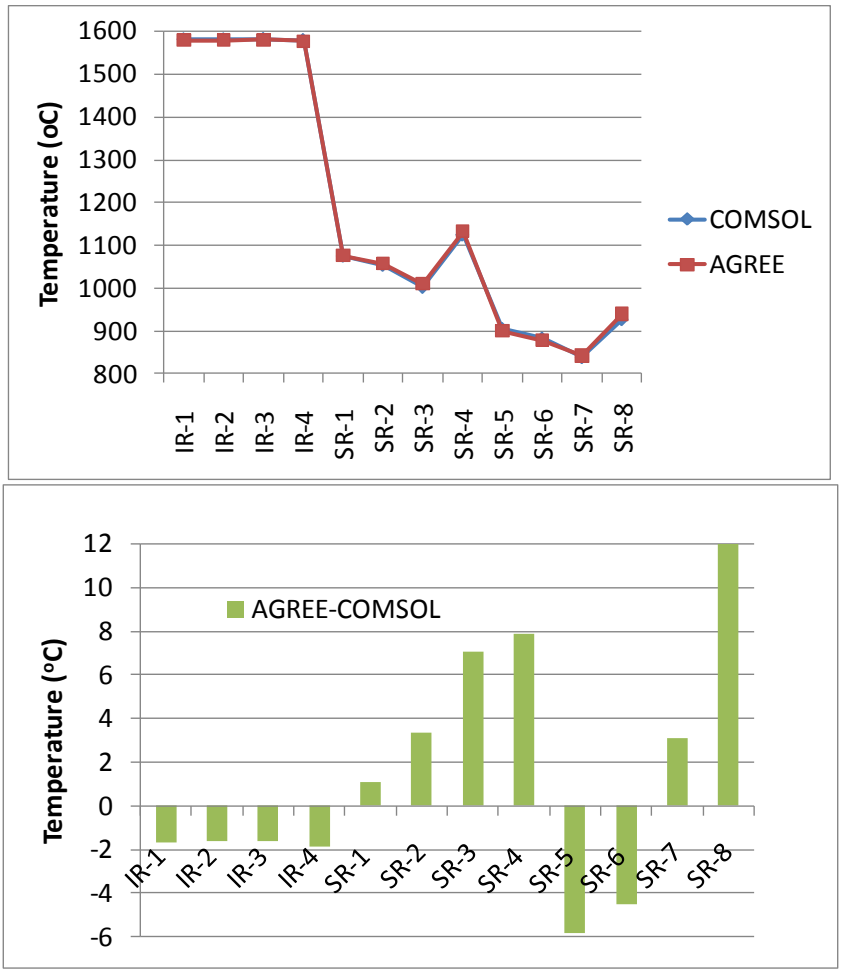

Fig. 12: Reflector Block Temperatures.

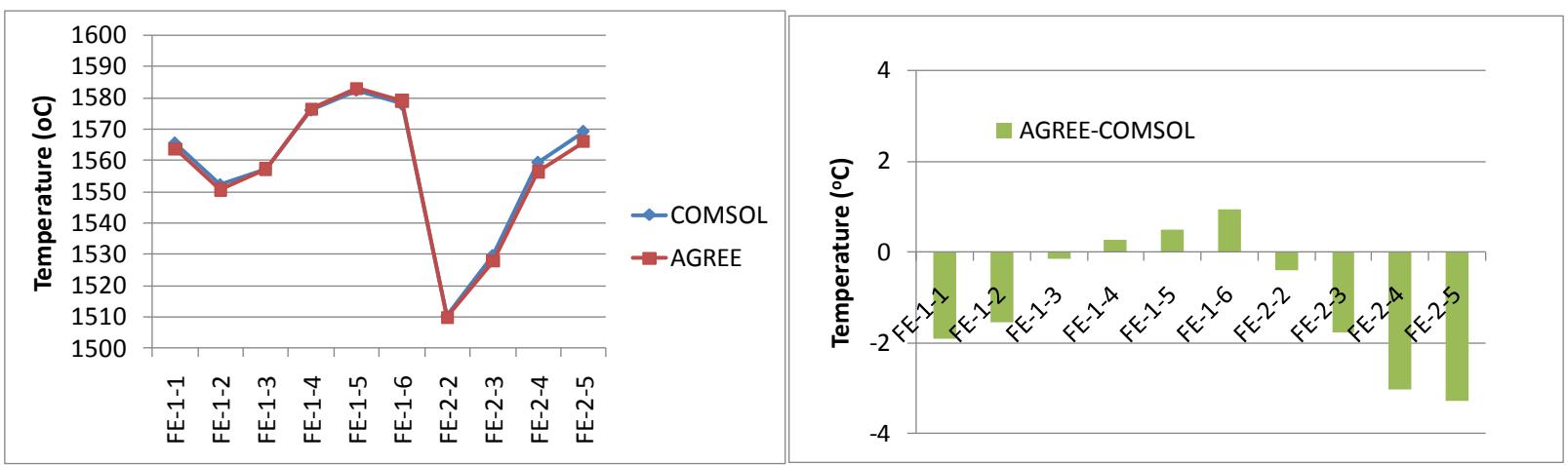



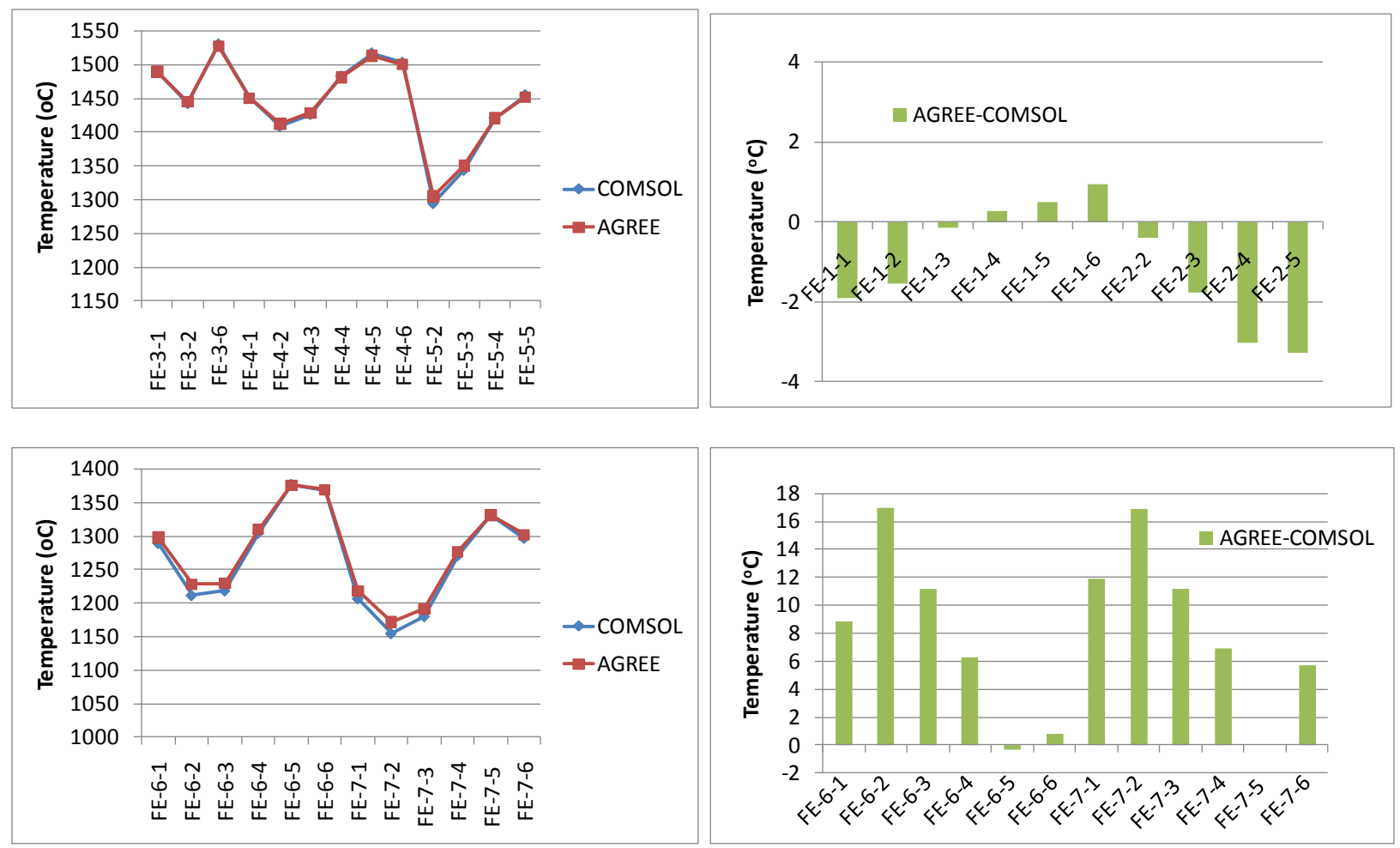

Fig. 13: Fuel Block Triangle Temperatures.

As it is seen from the comparisons, AGREE produces result that agree well with a very fine mesh COMSOL model. The COMSOL model requires $10.2 \mathrm{~GB}$ of memory and about 30 minutes runtime. On the other hand AGREE requires only a few megabytes of memory and runtime of a few seconds. 


\section{Chapter 4 Multiscale Methods for Hot Spot Prediction in the Prismatic HTR}

Studies performed on the prismatic HTGR show that information from a low-order diffusion solution passed to a smaller high-fidelity model is able to capture the appropriate higher-order spatial solution that is normally achieved with the full high-fidelity model. This work was motivated by the inability of the low-order solution to accurately approximate the hot spots within the prismatic core. Thus, it focused on the pin powers of a prismatic fuel assembly [1].

This study investigates the use of this methodology in a region of interest for the HTTR: the instrument/irradiation tubes where measurements were taken at core startup. This is a region near the core edge with three empty instrument tubes used to axially lower measurement equipment for startup measurements and future irradiations. Figure 1 highlights the location of one of these regions in the core.

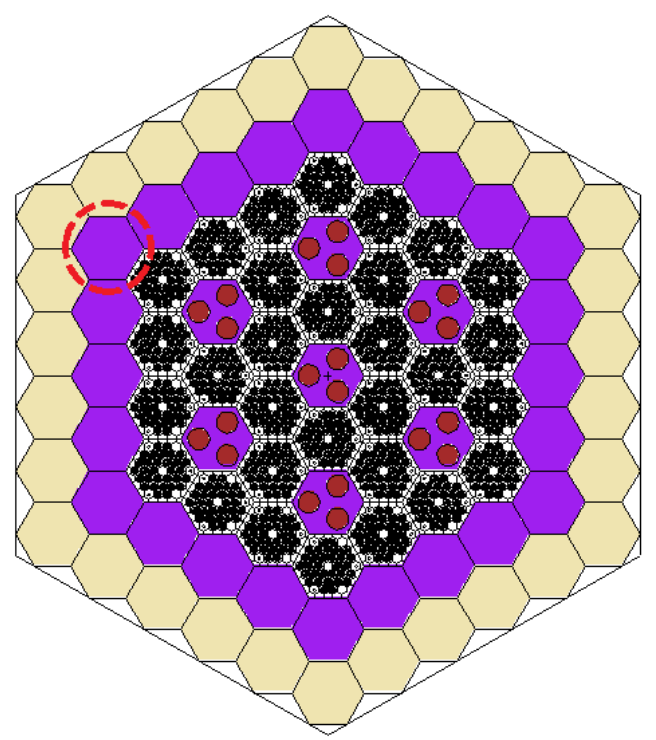

Figure 1. Location of the region of interest in the HTTR core. The instrument tubes are not shown. The other three-hole assemblies are control rod drive holes.

\section{Core Modeling and Multiscale Method}

We will use TRIPEN [2] to provide the low-order nodal solution and will pass information to a high-fidelity model in MCNP5 [3] by way of albedo boundary conditions. MCNP5 will then be run as a simple kcode problem. An alternative way of passing the necessary information by incident angular flux offers no better accuracy and requires significantly more work [1].

The albedo is defined as the ratio of the outgoing current to the incoming current. It is calculated as is traditionally done in diffusion theory:

$$
J_{g}^{o u t} \approx \phi_{g}+2 J_{g}^{n e t}
$$




$$
\begin{gathered}
J_{g}^{i n} \approx \phi_{g}-2 J_{g}^{\text {net }}, \\
\alpha_{g}=\frac{J_{g}^{\text {out }}}{J_{g}^{i n}} .
\end{gathered}
$$

Generally, the albedos form a matrix of size G X G, where G is the number of energy groups. Neutrons leaving in one energy group can reenter in another. However, we simplify the albedo matrix by only considering the diagonal elements; neutrons leave and reenter in the same energy group [1]. This simplifies the implementation of the albedo boundary condition in MCNP5.

We use the same TRIPEN 26 energy group structure in passing albedo boundary conditions to the MCNP5 model. The behavior of a neutron follows: upon leaving via an albedo boundary, its energy remains the same, its direction is changed according to a specular reflection, and its weight is augmented by the defined albedo for that boundary and group

$$
w^{\text {in }}=\alpha_{g} w^{\text {out }} .
$$

The albedo boundary conditions are defined on the boundaries of a smaller MCNP5 model. The volume between the region of interest and the albedo boundary condition is defined as the buffer region. In passing through this buffer region, neutrons theoretically should regain the high-order effects that are lost during the low-order approximation via use of the albedo boundary condition. Thus, the solution is expected to improve as the buffer region is enlarged.

Figure 2 shows a diagram of one assembly in the MCNP5 model. The core layout is as shown in Figure 1. Axially, the model has reflective boundary conditions on the top and bottom of a single assembly; an infinite axial lattice of assemblies. The TRIPEN model was built to reflect this model [4].

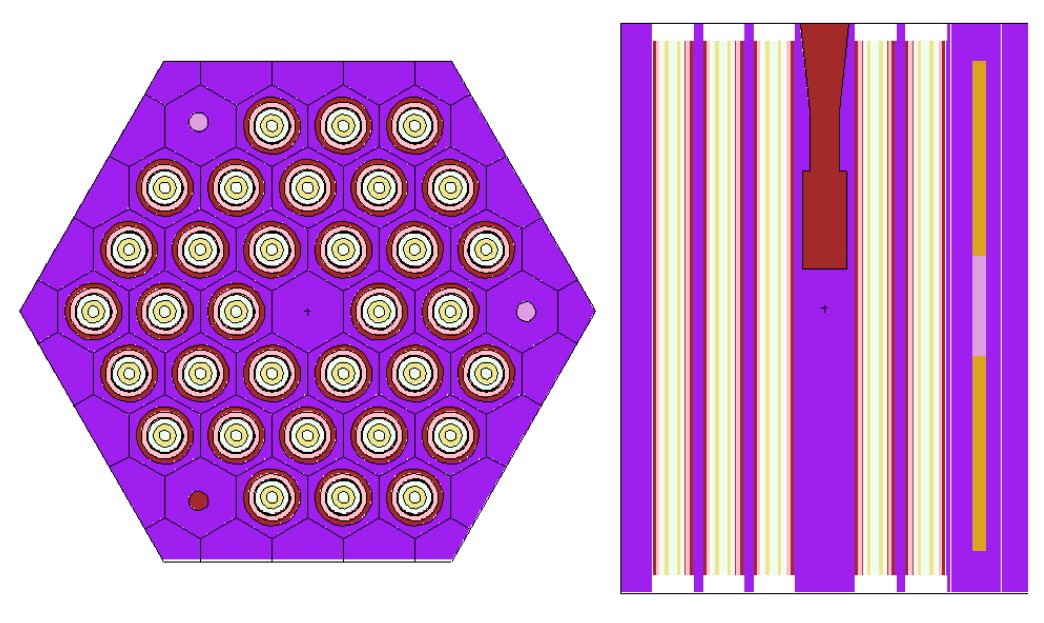

Figure 2. MCNP5 model of assembly radial (left) and axial (right) sections.

We ran both the full core high-fidelity MCNP5 model and the TRIPEN model for comparison. To define the buffer region necessary to achieve an accurate solution, we will vary it from $1 / 2$ to 
$1 \frac{1}{2}$ assembly lengths as shown in Figure 3. Eliminating the buffer region is not an option in this case because of the lack of fuel in our region of interest, and thus the lack of a source for the MCNP5 kcode calculation. Furthermore, in only the $1 / 2$-assembly length buffer region case are there 6 albedo boundary conditions. Due to the proximity of the region of interest the edge of the core, there are 2 partial albedo boundaries in the 1-assembly buffer region case and only 3 albedo boundaries in the $1 \frac{1}{2}$-assembly buffer region case. Note also that $1 \frac{1 / 2}{2}$ assembly lengths showed good results in the previous study [1] and it is expected that this large a buffer region should not be needed [5].
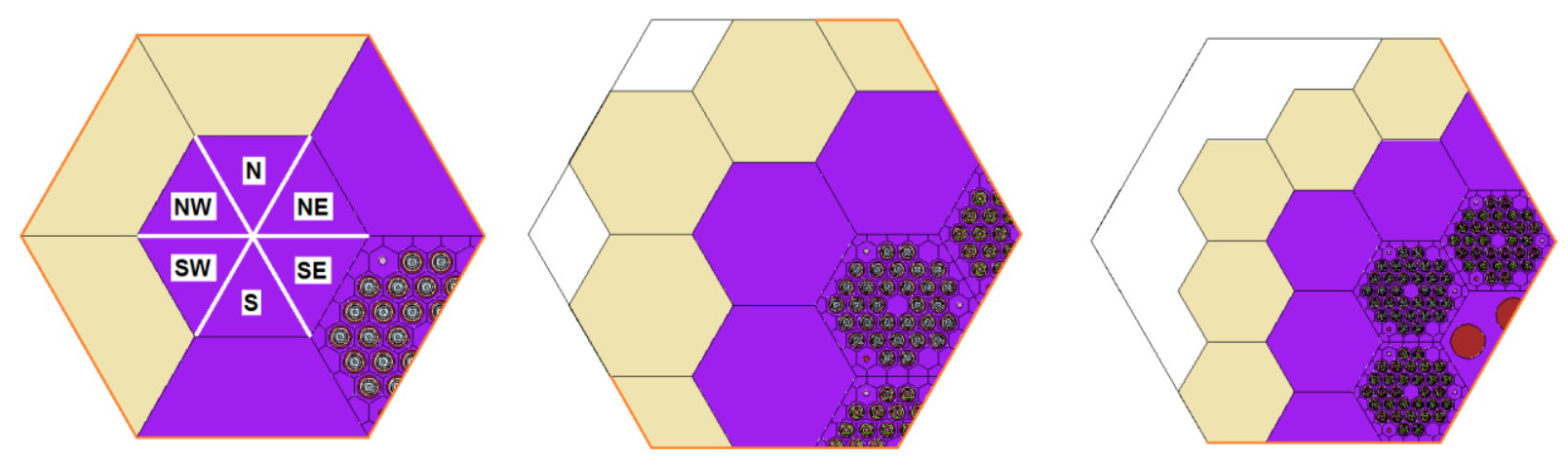

Figure 3. MCNP5 buffer regions around the assembly of interest: Half-ring (left), full ring (middle), and 1.5 rings (right). Boundaries defined as albedo emphasized. All other boundaries are vacuum.

The triangular geometry of TRIPEN divides each hexagonal fuel element into 6 pieces and divides the surfaces into pieces the side length of an assembly. Because of this, multiple albedos are obtained for each albedo boundary. These are not defined separately in MCNP5, but are averaged into one albedo boundary condition for each of the 26 energy groups for each parallel albedo boundary. Surface definitions in MCNP5 make implementing otherwise difficult. The MCNP5 flux tallies are adjusted to reflect the triangular mesh of TRIPEN for an apples-to-apples comparison as shown in Figure 3.

\section{Results}

First, we compare the overall flux values within each of the six triangular sections in within the region of interest. Table 1 gives the values and percent error from the full core MCNP5 model for each of these regions. Errors in the 11/2-assembly buffer region case are on the same order as (or 2-3 times larger than) the statistical uncertainty of the MCNP5 flux tallies. 
Table 1. The normalized flux distribution (\% deviation from the full core model) for the six sections.

\begin{tabular}{|c|c|c|c|c|c|c|c|c|c|}
\hline \multirow{3}{*}{$\begin{array}{l}\text { Section } \\
\text { SW }\end{array}$} & \multirow{2}{*}{\multicolumn{2}{|c|}{ TRIPEN }} & \multirow{3}{*}{\begin{tabular}{|l|}
$\begin{array}{l}\text { Full } \\
\text { Core }\end{array}$ \\
0.138
\end{tabular}} & \multicolumn{6}{|c|}{ Multiscale Buffer Regions } \\
\hline & & & & \multicolumn{2}{|c|}{$11 / 2$ assemblies } & \multicolumn{2}{|c|}{1 assembly } & \multicolumn{2}{|c|}{$1 / 2$ assembly } \\
\hline & 0.137 & $(-0.66)$ & & 0.137 & $(-0.24)$ & 0.138 & $(0.07)$ & 0.154 & $(12.1)$ \\
\hline NW & 0.113 & $(0$. & 0112 & & $(0$. & & & 0 & (26.8) \\
\hline $\mathrm{N}$ & & & & & $(-0$ & & $(0.96)$ & 0 & (13.2) \\
\hline $\mathrm{NE}$ & 92 & 10. & & 190 & $(-0$. & 0.1 & $(0.04)$ & 0.176 & $(-7.6)$ \\
\hline SE & 0.227 & $(-0.12)$ & 0.228 & 0.228 & $(0.22)$ & 0.227 & $(-0.41)$ & 0.194 & $(-14.8)$ \\
\hline $\mathrm{S}$ & 0.196 & $(-1.24)$ & 0.198 & 0.198 & $(0.04)$ & 0.197 & $(-0.77)$ & 0.182 & $(-8.18)$ \\
\hline
\end{tabular}

Table 1 shows the trend of increasing the buffer region to increase the accuracy of the solution. The $1 / 2$-assembly buffer region case does not provide the necessary accuracy. The results for the $11 / 2$-assembly buffer region case agree very well with the full core results, and the 1 -assembly buffer region case also agrees well.

To investigate where the $1 / 2$-assembly falls short of accurately describing the flux distribution, we compare the energy spectra of the five cases in Figure 4. We focus on the triangular regions furthest and closest to the core center, or adjacent to the outer reflector and fuel, respectively. The other triangular regions have similar spectra, thus, showing them would be redundant. These two triangular regions have the highest deviation for the $1 / 2$-assembly case model and their spectra are the most telling. 

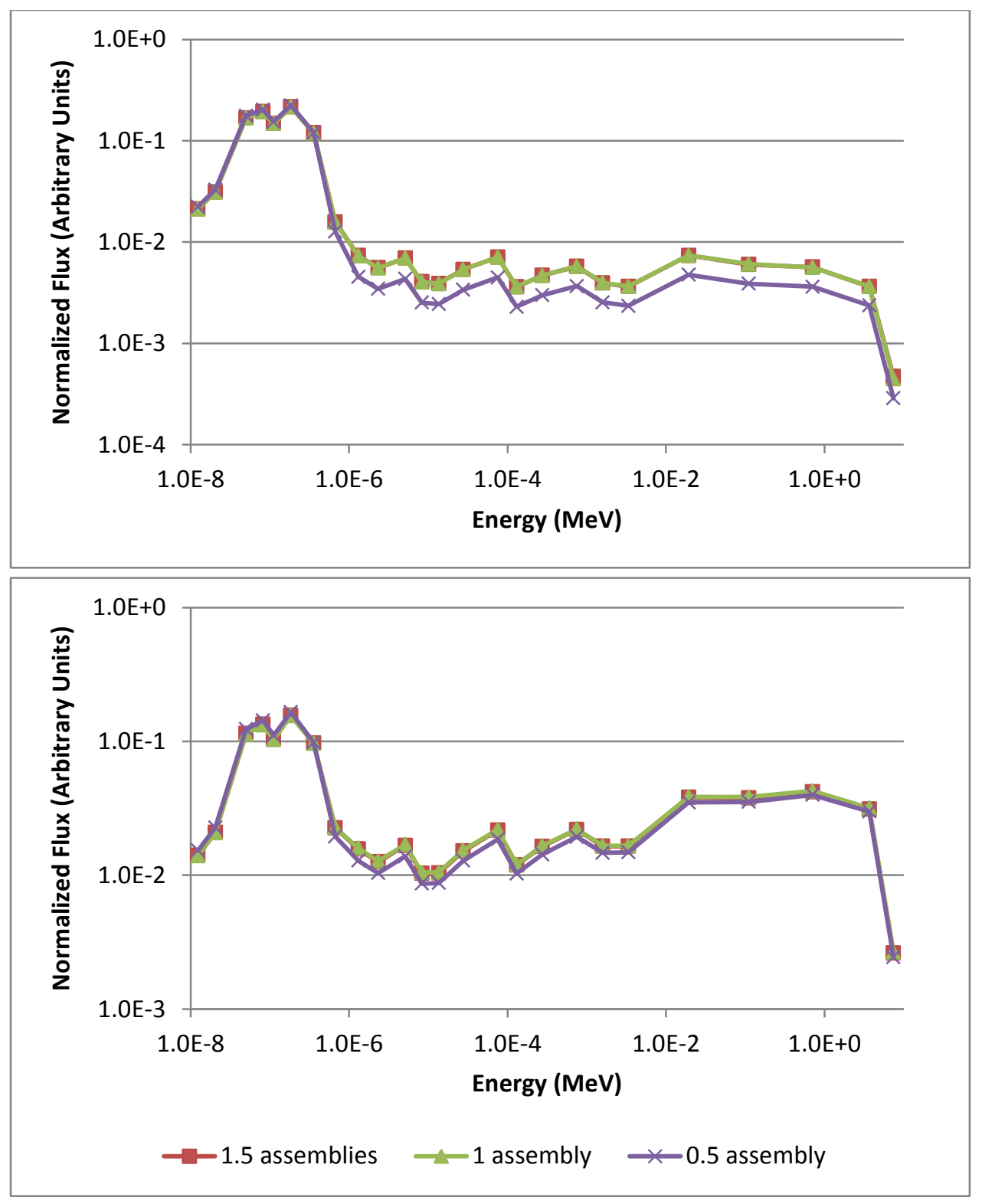

Figure 4. The spectra of the triangular section closest to the reflector (top) and fuel (bottom). On a plot of this scale the full core spectrum is indistinguishable from the 1.5 -assembly buffer region spectrum.

Regardless of the size of the buffer region, the shape of the thermal spectrum is approximated well. It is possible that the low-order approximation is able to regain the high-order information by slowing down in energy. This is likely aided by the fact that neutrons cannot scatter to nearzero energies in a collision with carbon as easily in comparison to a collision with a hydrogen nucleus. 
Upon inspection of the epithermal and fission energy area of the spectrum, it is evident why the $1 / 2$-assembly buffer region case is inaccurate. For the region closest to the fuel, the fast and thermal fluxes are approximated well, but the epithermal flux is underestimated. For the region closest to the reflector, both the fast and epithermal flux es are underestimated. This trend is

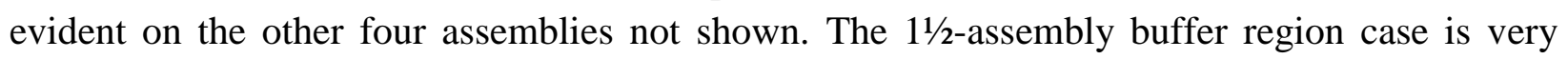
accurate and matches the full-core MCNP5 calculated flux. The 1-assembly buffer region case falls very close to the $1 \frac{1}{2}$-assembly case, performing well at approximating the flux spectrum. This supports our hypothesis that we will need a smaller buffer region than the pin-power case to approximate the flux in the region of interest well.

This study has been limited by the information provided about the HTTR [4] and can easily be extended to other areas of interest within the core, as well as using albedos on axial boundary conditions or obtaining axial flux distributions using radial-bounding albedos. 


\section{Chapter 5 Summary, Conclusion, and Recommendations for Future Work}

This report summarized the work performed in the project "Multi-Scale Multi-physics Methods Development for the Calculation of Hot-Spots in the NGNP". The first phase of the work investigated fundamental issues in multiscale neutronics in order to accurately describe the interface between higher order multigroup neutron transport and the lower order few neutron diffusion theory, which is used in standard NGNP safety calculations. Because a higher order non-orthogonal deterministic transport method was not available, this work was first performed in Cartesian geometry and the results were then later applied to the general geometry of the NGNP using Monte Carlo methods. The next phase of the work was to develop the multiphysics methods which provided the framework for the application of the work to practical NGNP reactor analysis. Specifically, the theory and methods implemented in the network flow, high temperature gas reactor dynamics code AGREE was described, to include the development of higher order Computational Fluids Dynamics and Heat Transfer models for the simulation of local fluids and heat transfer in the HTR fuel block. The final phase of the work was to develop and implement the multiscale neutronics methods for non-orthogonal geometries of the prismatic NGNP. Specifically, the methods were discussed in which Monte Carlo methods are used to predict of the local pin power based on the deterministic nodal solution in the PARCS code.

Based on the research performed here it is possible to more accurately predict the local hot spots in a prismatic HTR. Future work will be to automate these methods within the framework of AGREE/PARCS code system and to apply the methods to actual power reactor conditions such as the OECD MHTGR benchmark. 


\section{References}

[1] K. Koebke and M. Wagner, "The determination of the pin power distribution in a reactor core on the basis of nodal coarse mesh calculations," Atomkernenergie, vol. 30, p. 136, 1977.

[2] S. Grill, A. Jonsson, and J. Rec, "A nodal imbedded method to recover local power peaking from coarse mesh reactor calculations," Trans, vol. 35, p. 580, 1980.

[3] A. Jonsson, S. Grill, and J. R. Rec, "Nodal imbedded calculation for the retrieval of local power peaking from coarse mesh reactor analysis," in International Topical Meeting on Advances in Mathematical Methods for the Solution of Nuclear Engineering Problems, 1981.

[4] F. Nissen, Determination of Local Pin Powers in the Framework of NODAL Coarse-Mesh Solutions. PhD thesis, Riso National Laboratory, 1982.

[5] K. Smith, "Assembly homogenization techniques for light water reactor analysis," Progress in Nuclear Energy, vol. 17, pp. 303\{335, 1986.

[6] J. P. Jessee, W. A. Fiveland, L. H. Howell, P. Colella, and R. B. Pembe, "An adaptive mesh refinement algorithm for the radiative transport equation," JOURNAL OF COMPUTATIONAL PHYSICS, vol. 139, pp. 380\{398, 1998.

[7] Y. Wang, Adaptive Mesh Refinement Solution Techniques for the Multigroup Sn Transport Equation Using a Higher-Order Discontinuous Finite Element Method. PhD thesis, Texas A\&M University, 2009.

[8] C. Yi and A. Haghighat, "A hybrid block-oriented discrete ordinates and characteristics method algorithm for solving linear boltzmann equation," in Joint International Topical Meeting on Mathematics \& Computation and Supercomputing in Nuclear Applications (M\&C + SNA 2007), (Monterey, California), 2007.

[9] C. Yi and A. Haghighat, "A three-dimensional block-oriented hybrid discrete ordinates and characteristics method," Nuclear Science and Engineering, vol. 164, pp. $221\{247,2010$.

[10] B. Forget and F. Rahnema, "A spectral unfolding method," Transactions of the Americal Nuclear Society, vol. 1, pp. 669\{671, 2007. [11] L. Zhu and B. Forget, "A discrete generalized multigroup energy expansion theory," Nuclear Science and Engineering, vol. 166, pp. 239\{253, 2010.

[12] B. Forget and L. Zhu, "Mixed energy reactor sinulations using the discrete generalized multigroup method," in PHYSOR 2010 Advances in Reactor Physics to Power the Nuclear Renaissance, 2010.

[13] B. Forget, "Mixed energy methods." Personal Communication, 2011. 100

[14] H. R. Kim and N. Z. Cho, "Global/local iterative methods for equivalent diffusion theory parameters in nodal calculation," Annals of Nuclear Energy, vol. 20, pp. 767\{783, 1993.

[15] B. D. Ivanov, Methodology for Embedded Transport Core Calculation. PhD thesis, Pennsylvania State University, 2007.

[16] B. D. Ivanov, M. Ouisloumen, E. Mller, and K. N. Ivanov, "Embedded lattice transport calculations based on paragon-new code system for reactor core analysis," in International 
Conference on Reactor Physics, Nuclear Power: A Sustainable Resource, (Interlaken, Switzerland), 2008.

[17] B. D. Ivanov, E. Mller, M. Ouisloumen, and K. N. Ivanov, "Online homogenization technique facilitating nem-driven embedded lattice physics calculations," in International Conference on Reactor Physics, Nuclear Power: A Sustainable Resource, 2008.

[18] S. Zhang, C. Tang, H. Huang, and Y. Chao, "Feasibility of embedding nodal homogenization in next generation methods for $3 \mathrm{~d}$ pin-by-pin core simulation," in International Conference on Reactor Physics, Nuclear Power: A Sustainable Resource, 2008.

[19] K. Koebke, "A new approach to homogenization and group condensation," in Technical Committee Meeting on Homogenization Methods in Reactor Physics, (Lugano, Switzerland), 1978.

[20] K. Smith, Spatial Homogenization Methods for Light Water Reactor Analysis. PhD thesis, Massachusetts Institute of Technology, 1980.

[21] J. Y. Cho, H. G. Joo, K. S. Kim, S. Q. Zee, and M. H. Chang, "Three-dimensional heterogeneous whole core transport calculation employing planar moc solution," Transactions of the Americal Nuclear Society, vol. 87, p. 234, 2002.

[22] E. Lewis, M. A. Smith, N. Tsoulfanidis, G. Palmiotti, T. A. Taiwo, and R. N. Blomquist, "Benchmark specification for deterministic 2-d/3-d mox fuel assembly transport calculations without spatial homogenisation," tech. rep., Nuclear Energy Agency, 2001.

[23] B. Forget, "Transmittal of 33 group c5g7 library." Personal Communication, 2011.

[24] F. Rahnema, "Boundary condition perturbations in transport theory," Nuclear Science and Engineering, vol. 124, pp. 320\{326, 1996.

[25] F. Rahnema and E. M. Nichita, "Leakage corrected spatial (assembly) homogenization technique," Annals of Nuclear Energy, vol. 24, pp. 477\{488, 1997. 101

[26] F. Rahnema and P. Ravetto, "On the equivalence of boundary and boundary condition perturbations in transport theory and its diffusion approximation," Nuclear Science and Engineering, vol. 128, pp. 209\{223, 1998.

[27] M. S. McKinley and F. Rahnema, "Higher-order boundary condition perturbation theory for the diffusion approximation," Nuclear Science and Engineering, vol. 136, pp. 15\{33, 2000.

[28] M. S. McKinley and F. Rahnema, "High-order boundary condition perturbation theory for the neutron transport equation," Nuclear Science and Engineering,

vol. 140, pp. 285\{294, 2002.

[29] Y. Saad, Iterative Methods for Sparse Linear Systems. SIAM, 2003.

[30] Y. Saad, Numerical Methods for Large Eigenvalue Problems. Manchester University Press, 1992.

[31] Next Generation Nuclear Plant Licensing Strategy. A Report to Congress. Technical report, US Nuclear Regulatory Commision and the US Department of Energy, August 2008.

[32] Next Generation Nuclear Plant Phenomena Identification and Ranking Tables (PIRTs), Volume 1: Main Report. Technical Report NUREG/CR-6944, March 2008. 
[33] R.B. Vilim, W.D. Pointer, and T.Y.C. Wei. Prioritization of VHTR System Modeling Needs Based on Identification, Ranking, and Sensitivity Studies. Technical Report ANL-GenIV-071, April 2006.

[34] B.L. Strain. FLOPSY II a A Digital Computer Program for Calculating Flow and Pressure Drops in Hydraulic Networks. Technical Report KAPL-3030, 1965.

[35] R.B. Vilim. GAS-NET: A Two-Dimensional Network Code for Prediction of Core Flow and Temperature Distribution in the Prismatic Gas Reactor. In ICAPP 2007, Nice, France, May 13-18, 2007.

[36] R. Stainsby, A. Grief, and W. Worsley. Investigation of Local Heat Transfer Phenomena in a Prismatic Modular Reactor Core. Technical Report NR001/RP/001 R1, AMEC NSS Limited, March 30, 2009.

[37] J.P. Simoneau, J. Champigny, B. Mays, and L. Lommers. Three-dimensional simulation of the coupled convective, conductive, and radiative heat transfer during decay heat removal in an HTR. Nuclear Engineering and Design, 237:1923\{1937, 2007.

[38] W.D. Pointer, C.H. Lee, J.W. Thomas, Y.S. Jung, and W.S. Yang. Steadyt-State, WholeCore VHTR Simulation with Consistent Coupling of Neutronics and Thermo-uid Analysis. Technical Report ANL-GenIV-121, September 2009.

[39] S.V. Patankar. Numerical Heat Transfer and Fluid Flow. Taylor and Francis, 1980.

[40] T.J. Drzewiecki, V. Seker, and T.J. Downar. Software Requirements Specification Document, PMR Core Fluids Modeling in AGREE (Draft). Technical Report UM-NERS-10 0002, March 2010.

[41] M. Ishii and T. Hibiki. Thermo-Fluid Dynamics of Two-Phase Flow. Springer, 2006.

[42] RELAP5-3D Code Development Team. RELAP5-3D Code Manual Volume I: Code Structure, System Models, and Solution Methods. Technical Report INEEL-EXT-98-00834, June 2005.

[43] H. Petersen. The Properties of Helium: Density, Specific Heats, Viscosity, and Thermal Conductivity at Pressures from 1 to 100 bar and from Room Temperature to about $1800 \mathrm{~K}$. Technical Report Ris $\square$ Report No. 224, Danish Atomic Energy Commission, September 1970. [44] Reactor Core Design for High-Temperature Gas-Cooled Reactor, Part 1: Calculation of the Material Properties of Helium. Technical Report KTA 3102.1, Nuclear Safety Standards Commission (KTA), June 1978.

[45] S.W. Churchill. Friction factor equation spans all uid-ow regimes. Chemcial Engineering, 91, 1977.

[46] H.Kaburaki and T. Takizuka. Effect of Crossow on Flow Distribution on HTGR Core Column. Journal of Nuclear Science and Technology, 24:516\{525, 1987.

[47] S.J. Yoon, Y.J. Cho, K.Y. Kim, and M.H. Kim. Experimental Evaluation of the Bypass Flow in the VHTR Core. In Transcations of SMiRT 19, Toronto, Ontario, Cananda, August 12 17, 2007. 
[48] G.C. Park, S.J. Yoon, and C.Y. Jin. Fundamental Thermal-Fluid Experiment for Optimum Design of Very High Temperature Reactor. Technical Report KAERI/CM-1127/2008, Korea Atomic Energy Research Institute, April 2009.

[49] S.J. Yoon, C.Y. Yin, M.H. Kim, and G.C. Park. Experimental and Computational Assessment of Core Bypass Flow in Block-Type Very High Temperature Reactor. Nuclear Technology, 175:419, 2011.

[50] S.J. Yoon, C.Y. Yin, J.H. Lee, and Y.G. Lee. Study on the Flow Distribution in Prismatic VHTR Core with a Multi-Block Experiment and CFD Analysis. Nuclear Engineering aned Design, 241:5174, 2011.

[51] G.C. Park, S.J. Yoon, J.H Lee, and Y.G. Lee. Fundamental Thermal-Fluid Experiments for the Evaluation of the Bypass Flow in the VHTR Core. Technical Report KAERI/CM-1449/2010, Korea Atomic Energy Research Institute, December 2011.

[52] M.H. Kim et. al. Experimental and Analytic Study on the Core Bypass Flow in a Very High Temperature Reactor. Technical Report KAERI/RR-3293/2010, Korea Atomic Energy Research Institute, December 2011.

[53] S.J. Yoon, J.H. Lee M.H. Kim, and G.C. Park. The Effects of Crossow Gap and Axial Bypass Gap Distribution on the Flow Characteristics in Prismatic VHTR Core. Nuclear Engineering aned Design, 250:465\{479, 2012.

[54] N.I. Tak, M.M. Kim, H.S. Lim, J.M. Noh, T.J. Drzewiecki., and V. Seker.J. Downar. Validation of Numerical Methods to Calculate Bypass Flow in a Prismatic Gas-Cooled Reactor Core. In ICAPP 2013, Jeju Island, Korea, April 14-18, 2013.

[55] W.D. Pointer. CFD Predictions of Gap Bypass in Prismatic VHTR Cores. Transcations of the American Nuclear Society, 103:897, Las Vegas, Nevada, November 7-11, 2010.

[56] J.M. Noh et. al. Development of Very High Temperature Reactor Design Technology. Technical Report KAERI/RR-3462/2011, Korea Atomic Energy Research Institute, 2012.

[57] M.H. Kim and H.S. Lim. Evaluation of the Inuence of Bypass Flow Gap Distribution on the Core Hot Spot in Prismatic VHTR Core. Nuclear Engineering and Design, 241:3076, 2011.

[58] H.S. Lim and H.C. No. GAMMA Multidimensional Multicomponent Mixture Analysis to Predict Air Ingress Phenomena in HTGR. Nuclear Science and Engineering, 152:87\{97, 2006.

[59] M.H. Kim, H.S. Lim, and N.I. Tak. Computational Assessment of Bypass Flow in a MultiBlock Air Test. In Transactions of the Korean Nuclear Society Spring Meeting, Pyeongchang, Korea, May 27-28, 2010.

[60] R.B. Vilim. Coolant Distribution in the VHTR Prismatic Core. In ICAPP 2010, San Diego, CA, USA, Jun 13-17, 2010. 